\title{
Binary and Millisecond Pulsars at the New Millennium
}

\author{
Duncan R. Lorimer \\ Arecibo Observatory \\ HC3 Box 53995 \\ Puerto Rico 00612, U.S.A. \\ http://www.naic.edu/〜 dunc/ \\ dunc@naic.edu \\ Published on 18 June 2001 \\ www.livingreviews.org/Articles/Volume4/2001-5lorimer \\ Living Reviews in Relativity \\ Published by the Max Planck Institute for Gravitational Physics \\ Albert Einstein Institute, Germany
}

\begin{abstract}
We review the properties and applications of binary and millisecond pulsars. Our knowledge of these exciting objects has greatly increased in recent years, mainly due to successful surveys which have brought the known pulsar population to over 1300 . There are now 56 binary and millisecond pulsars in the Galactic disk and a further 47 in globular clusters. This review is concerned primarily with the results and spin-offs from these surveys which are of particular interest to the relativity community.

This article updates the Living Reviews article 1998-10 by Duncan Lorimer published on 25 September 1998.
\end{abstract}

(C)2001 Max-Planck-Gesellschaft and the authors. Further information on copyright is given at http://www.livingreviews.org/Info/Copyright/. For permission to reproduce the article please contact livrev@aei-potsdam.mpg.de. 


\section{Article Amendments}

On author request a Living Reviews article can be amended to include errata and small additions to ensure that the most accurate and up-to-date information possible is provided. For detailed documentation of amendments, please go to the article's online version at

http://www. livingreviews.org/Articles/Volume4/2001-5lorimer/.

Owing to the fact that a Living Reviews article can evolve over time, we recommend to cite the article as follows:

Lorimer, D.R.,

"Binary and Millisecond Pulsars at the New Millennium",

Living Rev. Relativity, 4, (2001), 5. [Online Article]: cited on <date>, http://www.livingreviews.org/Articles/Volume4/2001-5lorimer/.

The date in 'cited on <date>' then uniquely identifies the version of the article you are referring to. 


\section{Contents}

1 Preamble 5

1.1 What's new in this review? ............... 5

2 An Introduction to Pulsar Astronomy $\quad 7$

2.1 The lighthouse model . . . . . . . . . . . . . . . 7

2.2 Pulse profiles . . . . . . . . . . . . . . . . 8

2.3 The pulsar distance scale . . . . . . . . . . . . . . . . . 10

2.4 Normal and millisecond pulsars . . . . . . . . . . . . . . . 11

2.4.1 Spin parameters .............. . . 11

2.4.2 Binary companions .................. 13

2.4.3 Evolutionary scenarios . . . . . . . . . . . . . 14

2.4.4 Space velocities . . . . . . . . . . . . . . 17

2.5 Searching for pulsars . . . . . . . . . . . . . . . . 18

2.6 Where to look for binary and millisecond pulsars . . . . . . . . 18

2.6.1 All-sky searches . . . . . . . . . . . . . . . . . 19

2.6.2 Searches close to the plane of our Galaxy . . . . . . . . . 19

2.6.3 Searches at intermediate Galactic latitudes . . . . . . . . 20

2.6.4 Targeted searches of globular clusters . . . . . . . . . 20

2.7 Going further ................... 20

3 The Galactic Pulsar Population 22

3.1 Selection effects in pulsar searches . . . . . . . . . . . . . . 22

3.1.1 The inverse square law and survey thresholds . . . . . . . 22

3.1.2 Pulse dispersion and scattering . . . . . . . . . . . 24

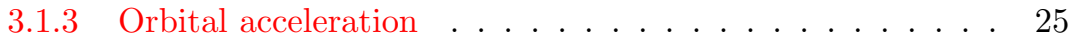

3.2 Correcting the observed pulsar sample . . . . . . . . . . . 27

3.2.1 Scale factor determination . . . . . . . . . . . . . 27

3.2.2 The small-number bias . . . . . . . . . . . . . . . 28

3.2.3 The beaming correction ................. . . 28

3.3 The population of normal and millisecond pulsars . . . . . . . . 29

3.3.1 Luminosity distributions and local number estimates . . . 29

3.3.2 Galactic population and birth-rates. . . . . . . . . . . . 31

3.3.3 Implications for gravitational wave detectors . . . . . . . 31

3.4 The population of relativistic binaries . . . . . . . . . . . . 32

3.4.1 Double neutron star binaries . . . . . . . . . . . . 32

3.4.2 White dwarf-neutron star binaries . . . . . . . . . . . . . 34

3.5 Going further . . . . . . . . . . . . . . 35

4 Pulsar Timing $\quad 36$

4.1 Observing basics ..................... 36

4.2 The timing model . . . . . . . . . . . . . . . . 37

4.3 Timing stability . . . . . . . . . . . . . . . . . . . . 40

4.4 Binary pulsars and Kepler's laws . . . . . . . . . . . . . . . . . . 43

4.5 Post-Keplerian parameters . . . . . . . . . . . . . . . 43 
4.6 Geodetic precession . . . . . . . . . . . . . . . . 47

4.7 Going further $\ldots \ldots \ldots \ldots \ldots \ldots$

5 Pulsars as Gravitational Wave Detectors $\quad 51$

5.1 Limits from individual pulsars . . . . . . . . . . . . . . . 51

5.2 A pulsar timing array . . . . . . . . . . . . . . 52

5.3 Going further . . . . . . . . . . . . . . . . 55

6 Summary and Future Prospects $\quad 56$

6.1 Acknowledgments . . . . . . . . . . . . . . . 57

7 Appendix: Tables of Binary and Millisecond Pulsars $\quad 58$

Living Reviews in Relativity (2001-5)

http://www. livingreviews .org 


\section{Preamble}

In the 34 years that have elapsed since the discovery [97] of pulsars, rapidly rotating highly magnetised neutron stars, the study of these fascinating objects has resulted in many applications in physics and astronomy. Striking examples include the confirmation of the existence of gravitational radiation [241] as predicted by general relativity [239, 240] and the first detection of an extra-solar planetary system $[273,186]$.

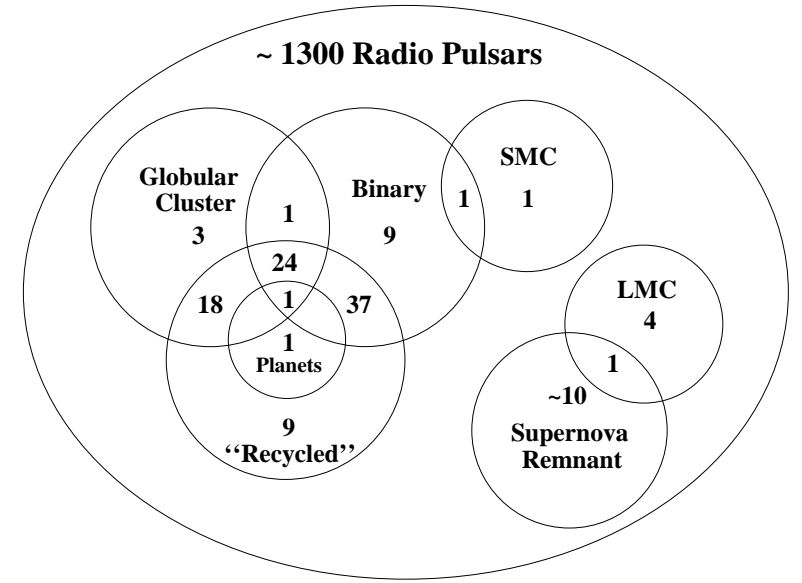

Figure 1: The numbers and locations of the various types of radio pulsars known as of December 2000. The large and small Magellanic clouds are denoted by $L M C$ and $S M C$.

The diverse zoo of radio pulsars currently known is summarized graphically by the Venn diagram in Fig. 1. Many new binary systems containing neutron stars are being discovered as a result of the latest generation of pulsar surveys. This review is concerned primarily with the results and spin-offs from these surveys which are of particular interest to the relativity community.

\subsection{What's new in this review?}

Since the first version of this article was written back in 1997/8 [128] a number of pulsar surveys using the Parkes radio telescope [157] have discovered almost 700 pulsars. As a result, the sample size is now double what it was in 1997. Many of the exciting new discoveries from these searches are discussed in this review. Up-to-date tables of parameters of binary and millisecond pulsars are included as an appendix. Several new sections/figures have been added and existing sections reworked and modularized to make the review more self-contained and (hopefully!) easier to read in an html setting. We begin in $\S 2$ with an overview of the pulsar phenomenon, the key observed population properties, the origin and evolution of pulsars and an introduction to pulsar search techniques. In

Living Reviews in Relativity (2001-5)

http://www.livingreviews.org 
$\S 3$, we review present understanding in pulsar demography, discussing selection effects and the techniques used to correct for them in the observed sample. This leads to robust estimates of the total number of normal and millisecond pulsars $(\S 3.3)$ and relativistic binaries ( $\$ 3.4)$ in the Galaxy and has implications for the detection of gravitational radiation from these systems. We discuss pulsar timing in $\S 4$. One application of these exceptional clocks, a sensitive detector of long-period gravitational waves, is discussed in $\S 5$. We conclude with a brief outlook to the future in $\S 6$.

Living Reviews in Relativity (2001-5)

http://www.livingreviews.org 


\section{An Introduction to Pulsar Astronomy}

Many of the basic observational facts about radio pulsars were established shortly after their discovery [97] by Bell and Hewish in 1967. In the intervening years, theoretical and observational progress has flourished. Although there are many remaining questions, particularly about the emission mechanism, the basic model has long been established beyond all reasonable doubt, viz.: Pulsars are rapidly rotating, highly magnetised neutron stars formed during the supernova explosions of massive $\left(\sim 5-10 M_{\odot}\right)$ stars. In the following, we discuss the basic observational properties most relevant to this review.

\subsection{The lighthouse model}

An animation showing the rotating neutron star or "lighthouse model" of the basic pulsar phenomenon is shown in Fig. 2.
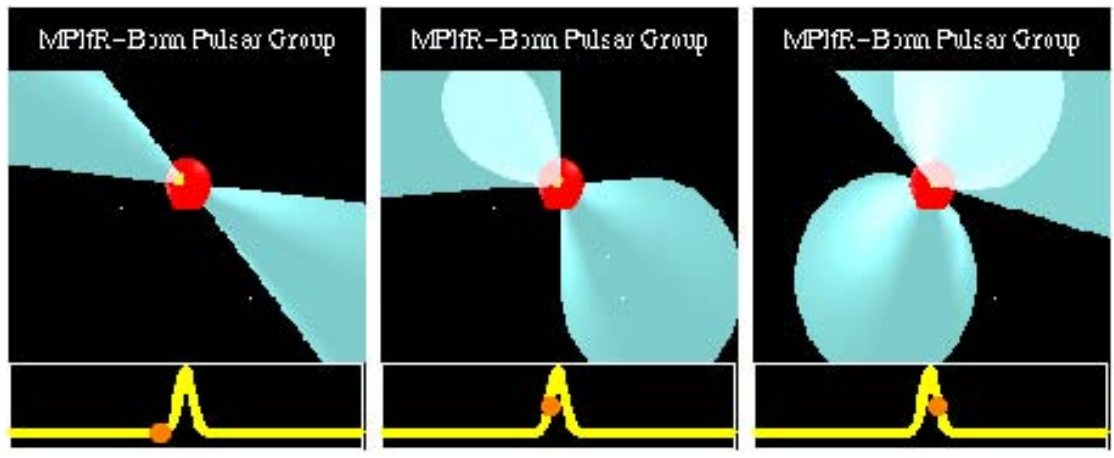

Figure 2: The rotating neutron star (or "lighthouse") model for pulsar emission. To see the movie in action go to the online version of this article on http://www.livingreviews.org/Articles/Volume4/2001-5lorimer. Animation designed by Michael Kramer.

As the neutron star spins, charged particles are accelerated out along magnetic field lines in the magnetosphere (depicted by the light blue cones). This acceleration causes the particles to emit electromagnetic radiation, most readily detected at radio frequencies as a sequence of observed pulses produced as the magnetic axis (and hence the radiation beam) crosses the observer's line of sight each rotation. The repetition period of the pulses is therefore simply the rotation period of the neutron star. The moving "tracker ball" on the pulse profile in the animation shows the relationship between observed intensity and rotational phase of the neutron star.

Neutron stars are extremely stable rotators. They are essentially large celestial flywheels with moments of inertia $\sim 10^{45} \mathrm{~g} \mathrm{~cm}^{2}$. The rotating neutron star model, independently developed by Pacini and Gold in 1968 [184, 85], predicts a gradual increase in the pulse period as the outgoing radiation carries 
away rotational kinetic energy. This model became universally accepted when a period increase of 36.5 ns per day was measured for the pulsar in the Crab nebula [202], enabling Gold [86] to show that a rotating neutron star with a large magnetic field must be the dominant energy supply for the nebula.

\subsection{Pulse profiles}

Pulsars are weak radio sources. Measured flux densities, usually quoted in the literature for a radio frequency of $400 \mathrm{MHz}$, vary between 0.1 and $5000 \mathrm{mJy}$ ( $1 \mathrm{Jy}$ $\equiv 10^{-26} \mathrm{~W} \mathrm{~m}^{-2} \mathrm{~Hz}^{-1}$ ). This means that, even with a large radio telescope, the coherent addition of many thousands of pulses is required in order to produce an integrated profile. Remarkably, although the individual pulses vary dramatically from pulse to pulse, at any particular observing frequency the integrated profile is very stable. The pulse profile can thus be thought of as a fingerprint of the emission beam.

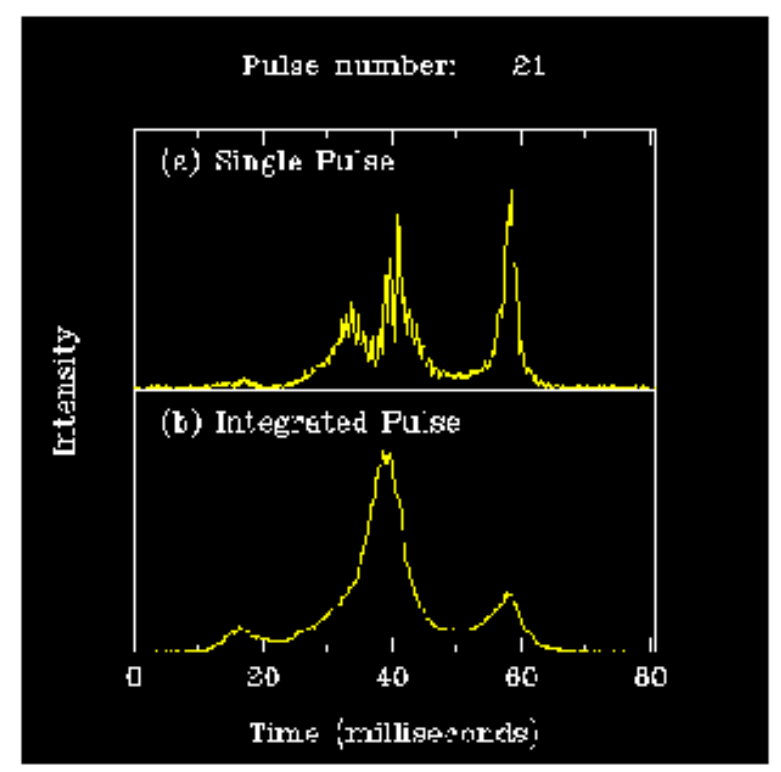

Figure 3: Single pulses from PSR B0329+54. To see the movie in action go to the online version of this article on http://www.livingreviews.org/ Articles/Volume4/2001-5lorimer.

The animation in Fig. 3 shows a sequence of consecutive single pulses from PSR B0329+54 $4^{1}$, one of the brightest pulsars. This pulsar is seen in the animation to stabilise into its characteristic 3-component form after the summation

\footnotetext{
${ }^{1}$ Pulsars are named with a PSR prefix followed by a "B" or a "J" and their celestial coordinates. Those pulsars discovered prior to 1990 are usually referred to by their "B" names (Besselian 1950 system). More recent discoveries are only referred to by their "J" names (Julian 2000 system).
}

Living Reviews in Relativity (2001-5)

http://www.livingreviews.org 
of a number of seemingly erratic single pulses. Stabilization time-scales are typically several hundred pulses [94]. This property is of key importance in pulsar timing measurements discussed in detail in $\S 4$.

Fig. 4 shows the rich diversity in morphology from simple single-component profiles to examples in which emission is observed over the entire pulse. The astute reader will notice two examples of "interpulses" - a secondary pulse separated by about 180 degrees from the main pulse. The most natural interpretation for this phenomenon is that the two pulses originate from opposite magnetic poles of the neutron star (see however [158]). Since this is an unlikely viewing angle we would expect interpulses to be a rare phenomenon. Indeed this is the case: the fraction of known pulsars in which interpulses are observed in their pulse profiles is only a few percent.

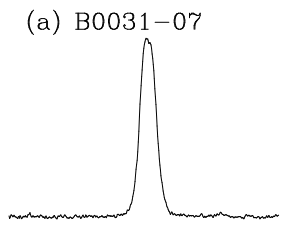

(d) B0525+21

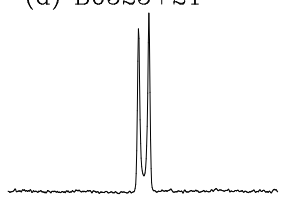

(g) B1937+21

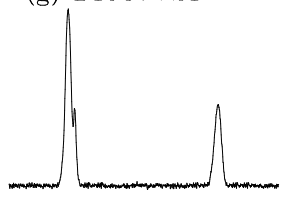

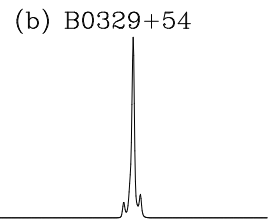

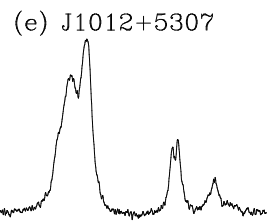

(h) J2124-3358

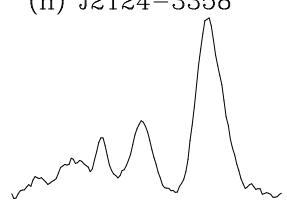

(c) J0437-4715

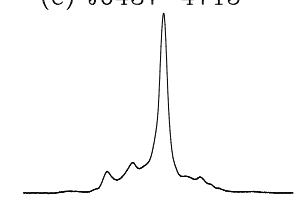

(f) B1831-04

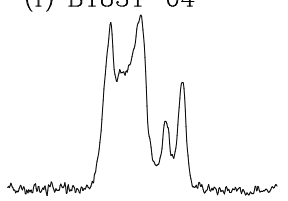

(i) J2145-0750

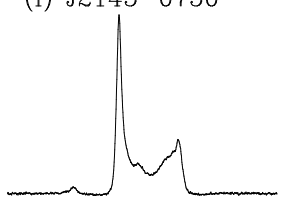

Figure 4: A variety of integrated pulse profiles taken from the available literature. References: (a, b, d, f: [87]); (c: [20]); (e, g, i: [122]); (h: [26]). Each profile represents 360 degrees of rotational phase. These profiles are part of a database of over 2600 multi-frequency pulse profiles for over 600 pulsars that is available on-line [164].

Two contrasting phenomenological models to explain the observed pulse shapes are shown in Fig. 5. The "core and cone" model, proposed by Rankin [198], depicts the beam as a core surrounded by a series of nested cones. Alternatively, the "patchy beam" model, championed by Lyne and Manchester [149, 89], has the beam populated by a series of randomly-distributed emitting regions. Further work in this area, particularly in trying to quantify the variety of pulse shapes (number of distinct components and the relative fraction that they oc- 

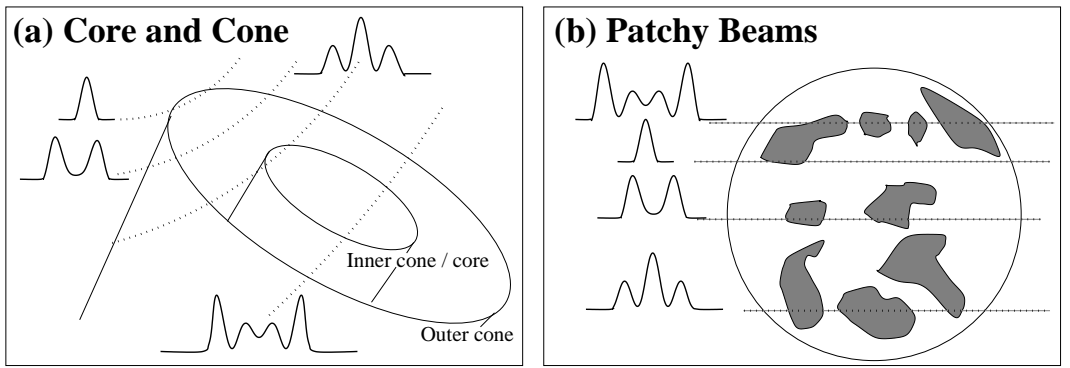

Figure 5: Phenomenological models for pulse shape morphology produced by different line-of-sight cuts of the beam (Figure designed by M. Kramer and A. von Hoensbroech).

cur) is necessary to improve our understanding of the fraction of sky covered by the radio pulsar emission beam. We return to this topic in the context of pulsar demography later on in $\S 3.2$.

\subsection{The pulsar distance scale}

From the sky distribution shown in Fig. 6 it is immediately apparent that pulsars are strongly concentrated along the Galactic plane. This indicates that pulsars populate the disk of our Galaxy. Unlike most other classes of astrophysical objects, quantitative estimates of the distances

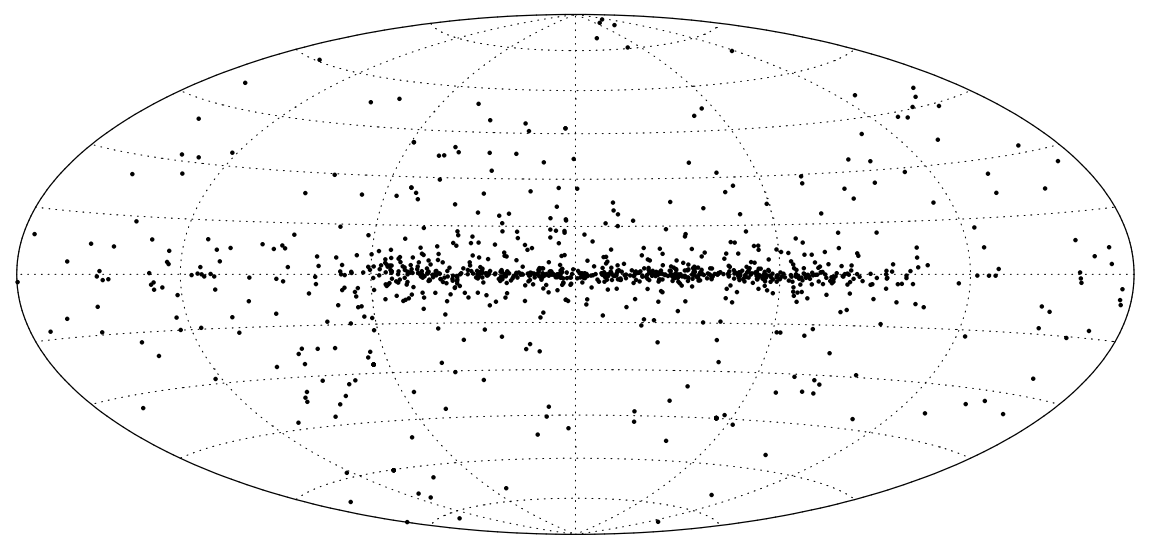

Figure 6: The sky distribution of 1026 pulsars in Galactic coordinates. The plane of the Galaxy is the central horizontal line. The Galactic centre is the midpoint of this line.

to each pulsar can be made from an effect known as pulse dispersion, the delay in pulse arrival times across a finite bandwidth. Dispersion occurs because the group velocity of the pulsed radiation through the ionised component of

Living Reviews in Relativity (2001-5)

http://www.livingreviews.org 
the interstellar medium is frequency dependent: pulses emitted at higher radio frequencies travel faster through the interstellar medium, arriving earlier than those emitted at lower frequencies. The delay $\Delta t$ in arrival times between a high frequency $\nu_{\mathrm{hi}}$ and a low frequency $\nu_{\text {lo }}$ pulse is given [156] by

$$
\Delta t=4150 \mathrm{~s} \times\left(\nu_{\mathrm{lo}}^{-2}-\nu_{\mathrm{hi}}^{-2}\right) \cdot \mathrm{DM},
$$

where the frequencies are in $\mathrm{MHz}$ and the dispersion measure $\mathrm{DM}\left(\mathrm{cm}^{-3} \mathrm{pc}\right)$ is the integrated column density of free electrons along the line of sight:

$$
\mathrm{DM}=\int_{0}^{d} n_{\mathrm{e}} d l .
$$

Here, $d$ is the distance to the pulsar (pc) and $n_{\mathrm{e}}$ is the free electron density $\left(\mathrm{cm}^{-3}\right)$. From Equation (2) it is obvious that a measurement of the delay across a finite bandwidth yields the DM. Pulsars at large distances have higher column densities and therefore larger DMs than those pulsars closer to Earth so that, from Equation (1), the dispersive delay across the bandwidth is greater. Hence, given the DM, the distance can be estimated from a model of the Galactic distribution of free electrons.

The electron density model is calibrated from the pulsars with independent distance estimates and measurements of scattering for lines of sight towards various Galactic and extragalactic sources. Independent distance estimates now exist for over 100 pulsars based on three basic techniques: neutral hydrogen absorption, trigonometric parallax (measured either with an interferometer or through pulse time-of-arrival techniques) and from associations with objects of known distance (i.e. supernova remnants, globular clusters and the Magellanic Clouds). Based on these data, Taylor \& Cordes [235] have developed an electron density model which is free from large systematic trends and can be used to provide distance estimates with an uncertainty of $\sim 30 \%$. However, use of this model to estimate distances to individual pulsars may result in uncertainties by as much as a factor of two. This model is currently being refined following recent pulsar discoveries and independent distance and scattering measurements (J. Cordes, private communication).

\subsection{Normal and millisecond pulsars}

\subsubsection{Spin parameters}

The present public-domain catalogue, available on-line at Princeton [196], contains up-to-date parameters for 706 pulsars. Parameters for many of the new Parkes multibeam pulsar surveys are also available on-line [28, 197]. Most of these are "normal" in the sense that their pulse periods $P$ are of order one second and are observed to increase secularly at rates $\dot{P}$ of typically $10^{-15} \mathrm{~s} / \mathrm{s}$. A growing fraction of the observed sample are the so-called "millisecond pulsars", which have spin periods primarily in the range 1.5 and $30 \mathrm{~ms}$ and rates of slowdown $\lesssim 10^{-19} \mathrm{~s} / \mathrm{s}$. The first millisecond pulsar discovered, B1937+21 [17],

Living Reviews in Relativity (2001-5)

http://www.livingreviews.org 
with $P=1.5578 \mathrm{~ms}$, remains the most rapidly rotating neutron star presently known.

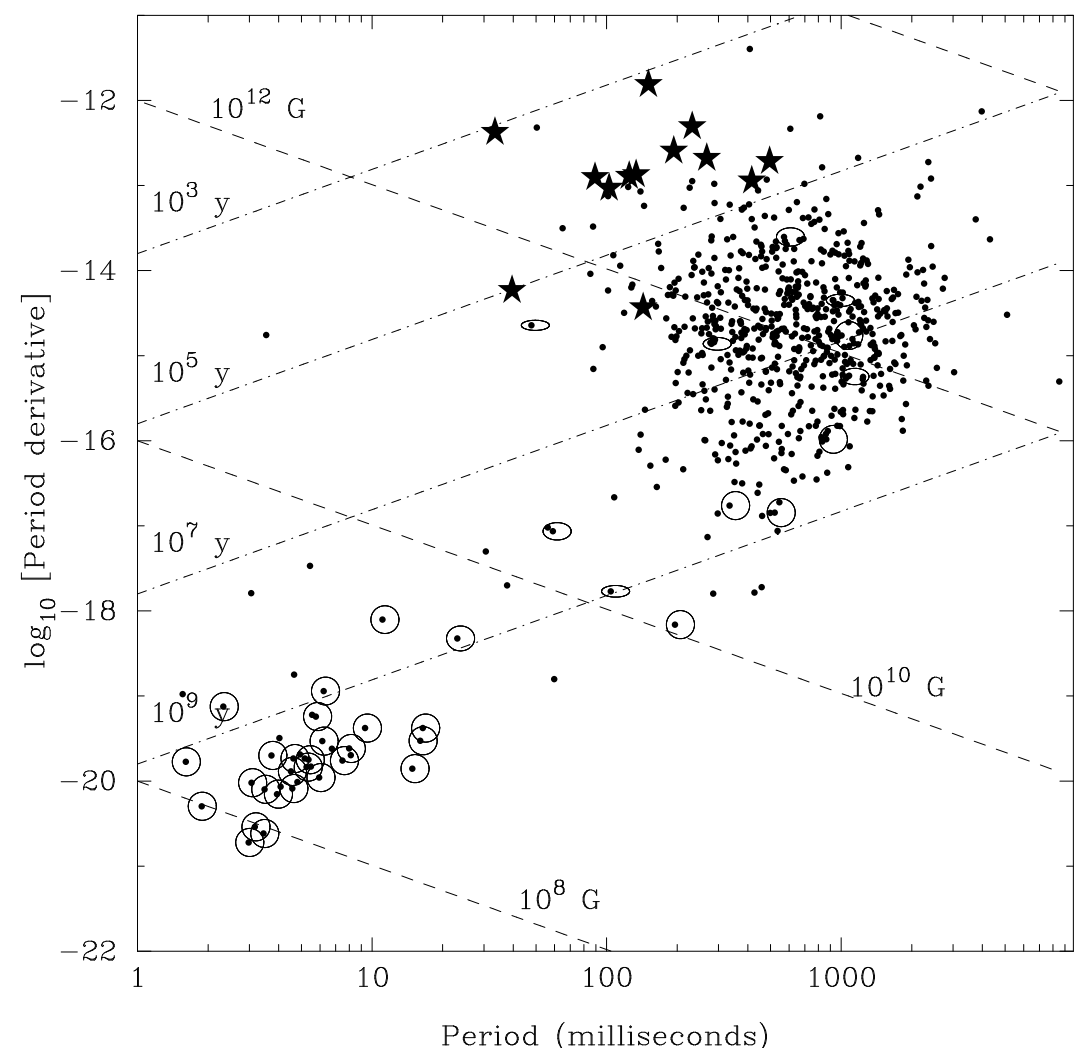

Figure 7: The ubiquitous $P-\dot{P}$ diagram shown for a sample of radio pulsars. Those objects known to be members of binary systems are highlighted by a circle (for low-eccentricity orbits) or an ellipse (for elliptical orbits). Pulsars thought to be associated with supernova remnants are highlighted by the starred symbols.

A very useful means of demonstrating the distinction between these two

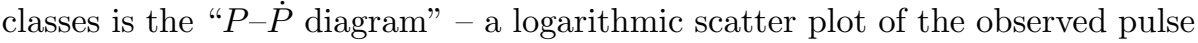
period versus the period derivative. As shown in Fig. 7, normal pulsars occupy the majority of the upper right hand part of the diagram, while the millisecond pulsars reside in the lower left hand part of the diagram. The differences in $P$ and $\dot{P}$ imply different ages and surface magnetic field strengths. By treating the pulsar as a rotating magnetic dipole, one may show that the surface magnetic field strength is proportional to $(P \dot{P})^{1 / 2}$ [163]. Lines of constant magnetic field strength are drawn on Fig. 7 , together with lines of constant characteristic age $\left(\tau_{\mathrm{c}}=P /(2 \dot{P})\right)$. Typical inferred magnetic fields and ages are $10^{12} \mathrm{G}$ and $10^{7} \mathrm{yr}$ for the normal pulsars and $10^{8} \mathrm{G}$ and $10^{9} \mathrm{yr}$ for the millisecond pulsars.

Living Reviews in Relativity (2001-5)

http://www.livingreviews.org 


\subsubsection{Binary companions}

As can be inferred from Fig. 1, just under $4 \%$ of all known pulsars in the Galactic disk are members of binary systems. Timing measurements ( $\S 4)$ place useful constraints on the masses of the companions which, supplemented by observations at other wavelengths, tell us a great deal about their nature. The present sample of orbiting companions are either white dwarfs, main sequence stars, or other neutron stars. Two notable hybrid systems are the "planet pulsars" PSR B1257+12 and B1620-26. B1257+12 is a 6.2-ms pulsar accompanied by at least three Earth-mass bodies [273, 186, 272] while B1620-26, an 11-ms pulsar in the globular cluster M4, is part of a triple system with a white dwarf and a high-mass planet [247, 15, 245].

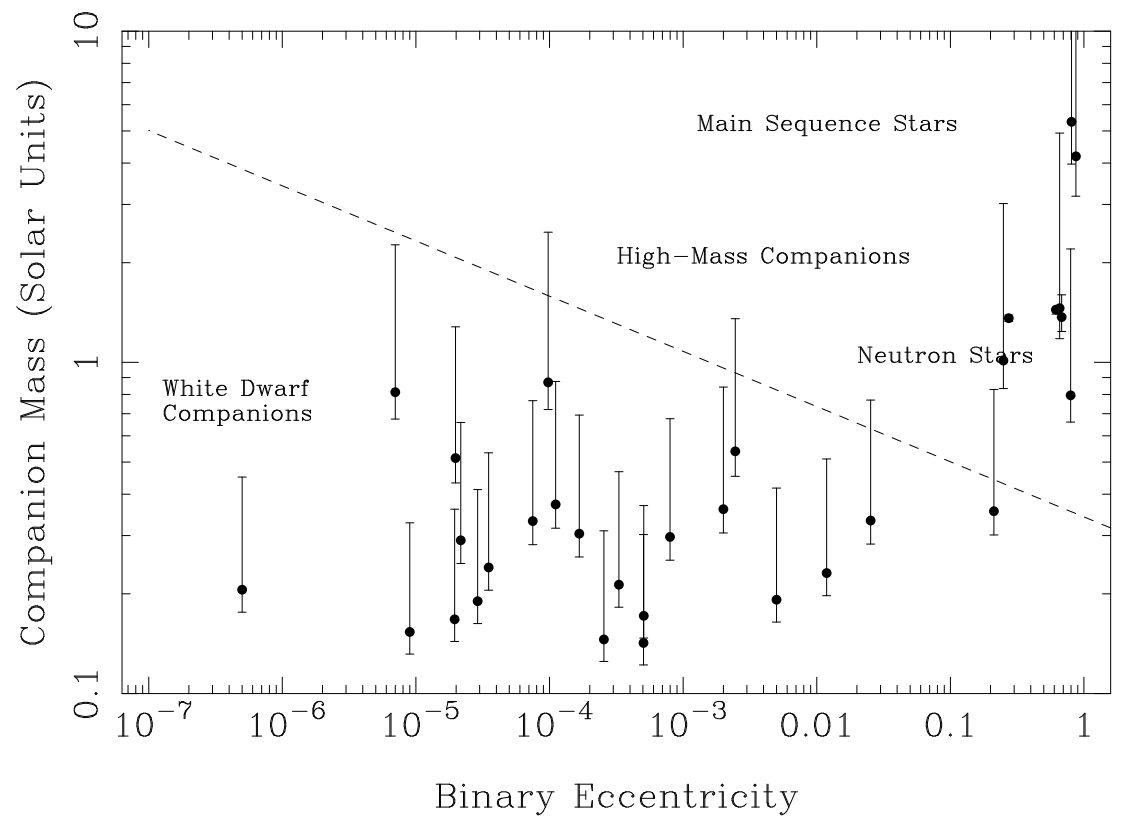

Figure 8: Companion mass versus orbital eccentricity for the sample of binary pulsars.

A very important additional difference between normal and millisecond pulsars is the presence of an orbiting companion. Orbital companions are much more commonly observed around millisecond pulsars ( $\sim 80 \%$ of the observed sample) than around the normal pulsars $(\lesssim 1 \%)$. Fig. 8 is a scatter plot of orbital eccentricity versus mass of the companion. The dashed line serves merely to guide the eye in this figure. Binary systems lying below the line are those with low-mass companions $\left(\lesssim 0.7 M_{\odot}\right.$ - predominantly white dwarfs $)$ and essentially circular orbits: $10^{-5} \lesssim e \lesssim 0.1$. Binary pulsars with high-mass companions $\left(\gtrsim 1 M_{\odot}-\right.$ neutron stars or main sequence stars) are in eccentric orbits, $0.15 \gtrsim e \gtrsim 0.9$, and lie above the line.

Living Reviews in Relativity (2001-5) http://www.livingreviews.org 


\subsubsection{Evolutionary scenarios}

The presently favoured model to explain the formation of the various types of systems has been developed over the years by a number of authors [34, 77, 218, 2]. The model is sketched in Fig. 9 and is now qualitatively summarised.

Starting with a binary star system, a neutron star is formed during the supernova explosion of the initially more massive star which has an inherently shorter main sequence lifetime. From the virial theorem it follows that the binary system gets disrupted if more than half the total pre-supernova mass is ejected from the system during the explosion [98, 31]. In addition, the fraction of surviving binaries is affected by the magnitude and direction of any impulsive "kick" velocity the neutron star receives at birth [98, 18]. Those binary systems that disrupt produce a high-velocity isolated neutron star and an OB runaway star [35]. The high binary disruption probability during the explosion explains, qualitatively at least, why so few normal pulsars have companions. Over the next $10^{7}$ yr or so after the explosion, the neutron star may be observable as a normal radio pulsar spinning down to a period $\gtrsim$ several seconds. After this time, the energy output of the star diminuishes to a point where it no longer produces significant radio emission.

For those few binaries that remain bound, and in which the companion is sufficiently massive to evolve into a giant and overflow its Roche lobe, the old spun-down neutron star can gain a new lease of life as a pulsar by accreting matter and therefore angular momentum at the expense of the orbital angular momentum of the binary system [2]. The term "recycled pulsar" is often used to describe such objects. During this accretion phase, the X-rays produced by the liberation of gravitational energy of the infalling matter onto the neutron star mean that such a system is expected to be visible as an X-ray binary system. Two classes of X-ray binaries relevant to binary and millisecond pulsars exist, viz. neutron stars with high-mass or low-mass companions. For a detailed review of the X-ray binary population, including systems likely to contain black holes rather than neutron stars, the interested reader is referred to [31].

The high-mass companions are massive enough to explode as a supernova, producing a second neutron star. If the binary system is lucky enough to survive this explosion, it ends up as a double neutron star binary. The classic example is PSR B1913+16 [102], a 59-ms radio pulsar with a characteristic age of $\sim 10^{8} \mathrm{yr}$ which orbits its companion every $7.75 \mathrm{hr}[239,240]$. In this formation scenario, PSR B1913+16 is an example of the older, first-born, neutron star that has subsequently accreted matter from its companion. So far there are no clear examples of systems where the second-born neutron star is observed as a radio pulsar. In the case of PSR B1820-11 [155], which may be an example, the mass of the companion is not well determined, so either a main-sequence [193] or a white dwarf companion [194] are plausible alternatives. This lack of observation of second-born neutron stars as radio pulsars is probably reasonable when one realises that the observable lifetimes of recycled pulsars are much larger than those of normal pulsars. As discussed in $\S 3.4 .1$, double neutron star binary systems are very rare in the Galaxy - another indication that the majority

Living Reviews in Relativity (2001-5)

http://www. livingreviews . org 


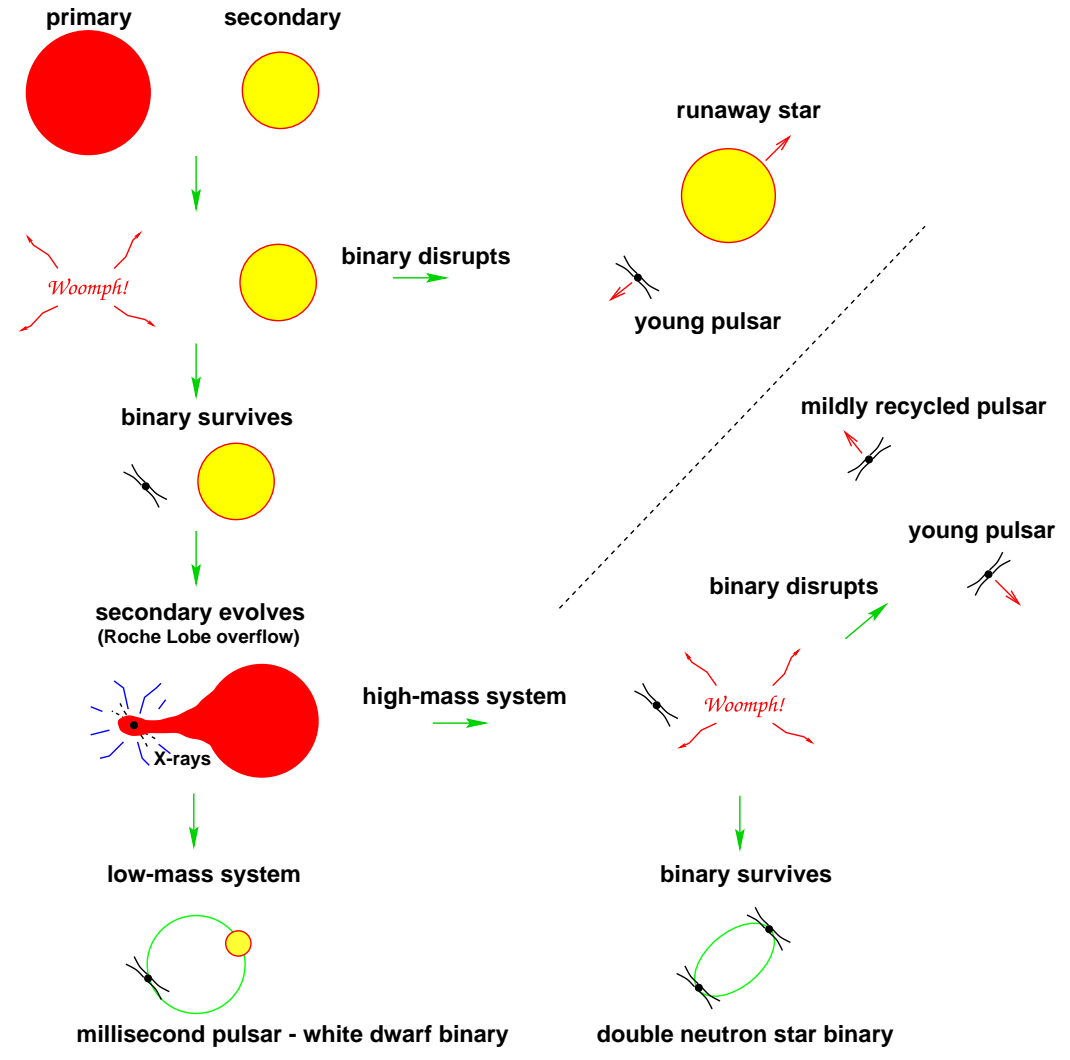

Figure 9: Cartoon showing various evolutionary scenarios involving binary pulsars.

Living Reviews in Relativity (2001-5) http://www.livingreviews.org 
of binary systems get disrupted when one of the components explodes as a supernova. Systems disrupted after the supernova of the secondary form a mildly-recycled isolated pulsar and a young pulsar formed during the explosion of the secondary.

Although no system has so far been found in which both neutron stars are visible as radio pulsars, timing measurements of three systems show that the companion masses are $1.4 M_{\odot}$ - as expected for neutron stars [215]. In addition, no optical counterparts are seen. Thus, we conclude that these unseen companions are neutron stars that are either too weak to be detected, no longer active as radio pulsars, or their emission beams do not intersect our line of sight. The two known young radio pulsars with main sequence companions massive enough to explode as a supernova probably represent the intermediate phase between high-mass X-ray binaries and double neutron star systems [110, 115].

The companions in the low-mass X-ray binaries evolve and transfer matter onto the neutron star on a much longer time-scale, spinning it up to periods as short as a few ms [2]. This model has gained strong support in recent years from the discoveries of quasi-periodic $\mathrm{kHz}$ oscillations in a number of low-mass X-ray binaries [266], as well as Doppler-shifted 2.49-ms X-ray pulsations from the transient X-ray burster SAX J1808.4-3658 [267, 53]. At the end of the spinup phase, the secondary sheds its outer layers to become a white dwarf in orbit around a rapidly spinning millisecond pulsar. Presently $\sim 10$ of these systems have compelling optical identifications of the white dwarf companion [25, 27, 139, 138]. Perhaps the best example is the white dwarf companion to the 5.25ms pulsar J1012+5307 [177, 133]. This 19th magnitude white dwarf is bright enough to allow measurements of its surface gravity and orbital velocity [257].

The range of white dwarf masses observed is becoming broader. Since this article originally appeared in 1998, the number of "intermediate-mass binary pulsars" [43] has grown significantly [49]. These systems are distinct to the "classical" millisecond pulsar-white dwarf binaries like PSR J1012+5307 in several ways: (1) the spin period of the radio pulsar is generally longer (9-200 ms); (2) the mass of the white dwarf is larger (typically close to $1 M_{\odot}$ ); (3) the orbital eccentricity, while still essentially circular, is often significantly larger $\left(\sim 10^{-3}\right)$. It is not presently clear whether these systems originated from either low- or high-mass X-ray binaries. It was suggested by van den Heuvel [254] that they have more in common with high-mass systems, the difference being that the secondary star was not sufficiently massive to explode as a supernova. Instead it formed a white dwarf. Detailed studies of this sub-population of binary pulsars are required for further understanding in this area.

Another relatively poorly understood area is the existence of solitary millisecond pulsars in the Galactic disk (which comprise just under 20\% of all Galactic millisecond pulsars). Although it has been proposed that the millisecond pulsars have got rid of their companion by ablation, as appears to be happening in the PSR B1957+20 system [83], it is not clear whether the time-scales for this process are feasible. There is some observational evidence that suggests that solitary millisecond pulsars are less luminous than binary millisecond pulsars $[20,122]$. If confirmed by future discoveries, this would need to be explained

Living Reviews in Relativity (2001-5)

http://www. livingreviews .org 
by any viable evolutionary model.

\subsubsection{Space velocities}

Pulsars have long been known to have space velocities at least an order of magnitude larger than those of their main sequence progenitors, which have typical values between 10 and $50 \mathrm{~km} \mathrm{~s}^{-1}$. The first direct evidence for large velocities came from optical observations of the Crab pulsar in 1968 [251], showing that the neutron star has a velocity in excess of $100 \mathrm{~km} \mathrm{~s}^{-1}$. Proper motions for about 100 pulsars have subsequently been measured largely by radio interferometric techniques [142, 21, 78, 92]. These data imply a broad velocity spectrum ranging from 0 to over $1000 \mathrm{~km} \mathrm{~s}^{-1}$ [148].

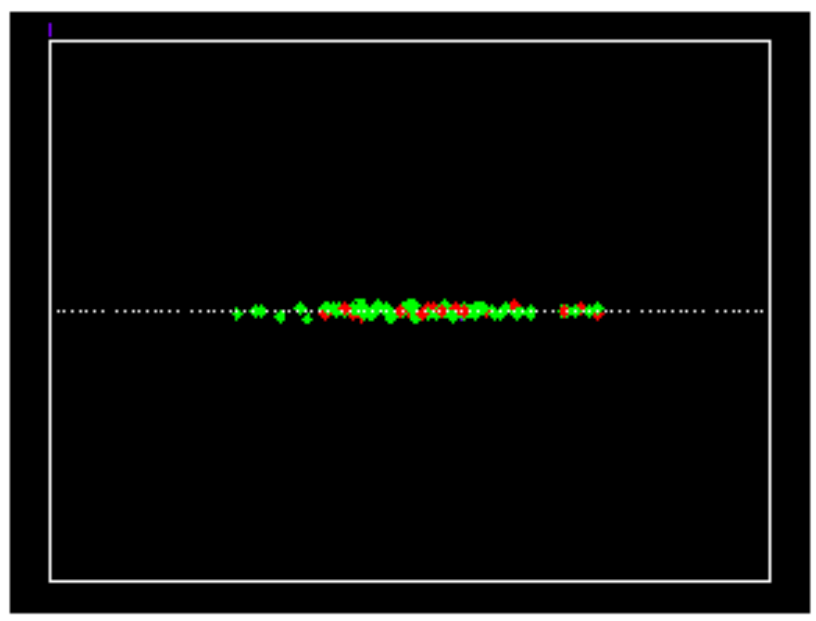

Figure 10: A simulation following the motion of 100 pulsars in a model gravitational potential of our Galaxy for 200 Myr. The view is edge-on, i.e. the horizontal axis represents the Galactic plane (30 kpc across) while the vertical axis represents $\pm 10 \mathrm{kpc}$ from the plane. This snapshot shows the initial configuration of young neutron stars. To see the movie in action go to the online version of this article on http://www.livingreviews.org/Articles/Volume4/20015lorimer.

Such large velocities are perhaps not surprising, given the violent conditions under which neutron stars are formed. Shklovskii [217] demonstrated that, if the explosion is only slightly asymmetric, an impulsive "kick" velocity of up to $1000 \mathrm{~km} \mathrm{~s}^{-1}$ is imparted to the neutron star. In addition, if the neutron star progenitor was a member of a binary system prior to the explosion, the pre-supernova orbital velocity will also contribute to the resulting speed of the newly-formed pulsar. High-velocity pulsars born close to the Galactic plane quickly migrate to higher Galactic latitudes. This migration is seen in Fig. 10, a dynamical simulation of the orbits of 100 neutron stars in a model of the Galactic gravitational potential. Given such a broad velocity spectrum, as much as half 
of all pulsars will eventually escape the gravitational potential of the Galaxy and end up in intergalactic space [148, 58].

Based on the proper motion data, recent studies have demonstrated that the mean birth velocity of normal pulsars is $\sim 450 \mathrm{~km} \mathrm{~s}^{-1}([148,132,58,84]$; see, however, also [93, 91]). This is significantly larger than the velocities of millisecond and binary pulsars. Recent studies suggest that their mean birth velocity is likely to be in the range $\sim 80-140 \mathrm{~km} \mathrm{~s}^{-1}[129,57,152]$. The main reason for this difference surely lies in the fact that about $80 \%$ of the millisecond pulsars are members of binary systems (§ 2.4) which could not have survived had the neutron star received a substantial kick velocity.

\subsection{Searching for pulsars}

Pulsar searching is, conceptually at least, a rather simple process - the detection of a dispersed, periodic signal hidden in a noisy time series collected using a large radio telescope. The search strategy is to form a large number of time series for different trial DM values. These data are then analysed for periodic signals. We give here a brief description of the basic search techniques. More detailed discussions can be found elsewhere [141, 178, 130].

A schematic pulsar search is shown in Fig. 11. The finite bandwidth is split up into a number of channels, typically using a filterbank or a correlator (see e.g. [13]), either of which usually provides a much finer frequency channelisation than the eight channels shown for illustrative purposes in Fig. 11. The channels are then de-dispersed (see $\S 3.1 .2$ ) to form a single noisy time series. An efficient way to find a periodic signal in these data is to take the Fast Fourier Transform (FFT) and plot the resulting amplitude spectrum. For a narrow pulse the spectrum will show a family of harmonics. To detect weaker signals still, a harmonic summing technique is usually implemented [141]. The best candidates are saved and the whole process is repeated for another trial DM.

After the data have been processed for a suitable range of DM, a list of pulsar candidates is compiled and the raw time series data are folded modulo each candidate period. In practise the analysis is often hampered by the presence of periodic interference sources which can often look very "pulsar-like". Although interference excision schemes (usually based on coincidence analyses of data taken from different points on the sky) work fairly well, interference is an everincreasing problem in radio astronomy and considerable efforts are required to carry out sensitive searches.

\subsection{Where to look for binary and millisecond pulsars}

Following the above discussions on demography and evolution, it is instructive to briefly summarize the rationale behind the major searches being carried out at the present time.

Living Reviews in Relativity (2001-5)

http://www.livingreviews.org 


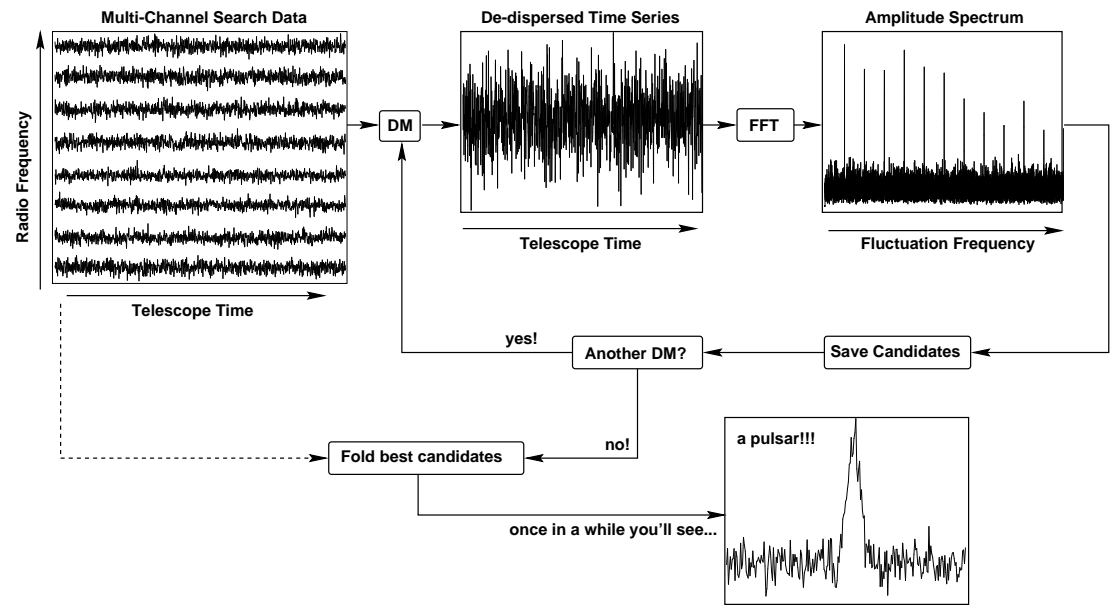

Figure 11: Schematic summarising the essential steps in a "standard" pulsar search.

\subsubsection{All-sky searches}

The oldest radio pulsars form a virialised population of stars oscillating in the Galactic gravitational potential. The scale height for such a population is at least $500 \mathrm{pc}$, about 10 times that of the massive stars which populate the Galactic plane. Since the typical ages of millisecond pulsars are several Gyr or more, we expect, from our vantage point in the Galaxy, to be in the middle of an essentially isotropic population of nearby sources. All-sky searches for millisecond pulsars at high Galactic latitudes have been very effective in probing this population. Much of the initial interest and excitement in this area was started at Arecibo when Wolszczan discovered two classic recycled pulsars at high latitudes: the neutron star binary B1534+12 [270] and the planets pulsar B1257+12 [273]. Surveys carried out at Arecibo, Parkes, Jodrell Bank and Green Bank by others in the 1990s found many other millisecond and recycled pulsars in this way. Camilo has written several excellent reviews of these surveys $[41,44,45]$. See also Tables 2,3 and 4 in the appendix.

\subsubsection{Searches close to the plane of our Galaxy}

Young pulsars are most likely to be found near to their place of birth, close to the Galactic plane. This is the target region of one of the Parkes multibeam surveys and has already resulted in the discovery of around 600 new pulsars [48, 159], almost half the number currently known! Such a large haul inevitably results in a number of interesting individual objects such as: PSR J1141-6545, a young pulsar in a relativistic 4-hr orbit around a white dwarf [116]; PSR J1740-3052, a young pulsar orbiting an $\sim 11 M_{\odot}$ star (probably a giant [159]); several intermediate-mass binary pulsars [49] and a likely double neutron star 
system, PSR J1811-1736 [147].

\subsubsection{Searches at intermediate Galactic latitudes}

In order to probe more deeply into the population of millisecond and recycled pulsars than possible at high Galactic latitudes, Edwards et al. have recently completed a survey of intermediate latitudes with the Parkes multibeam system $[74,75]$. The results of this survey are extremely exciting -58 new pulsars including 8 relatively distant recycled objects. Two of the new recycled pulsars from this survey recently announced by Edwards \& Bailes [75] are mildly relativistic neutron star-white dwarf binaries. An analysis of the full results from this survey should significantly improve our knowledge on the Galaxy-wide population and birth-rate of millisecond pulsars.

\subsubsection{Targeted searches of globular clusters}

Globular clusters have long been known to be breeding grounds for millisecond and binary pulsars. The main reason for this is the high stellar density in globular clusters relative to most of the rest of the Galaxy. As a result, lowmass X-ray binaries are almost 10 times more abundant in clusters than in the Galactic disk. In addition, exchange interactions between binary and multiple systems in the cluster can result in the formation of exotic binary systems. Since a single globular cluster usually fits well within a single telescope beam, deep targeted searches can be made. Once the DM of a pulsar is known in a globular cluster, the DM parameter space for subsequent searches is essentially fixed. This allows computation power to be invested in so-called acceleration searches for short-period binary systems (see $\S$ 3.1.3). To date, searches have revealed 47 pulsars in globular clusters (see Table 5 in the appendix for a list and the review by Kulkarni \& Anderson [123]). Highlights include the double neutron star binary in M15 [195] and a low-mass binary system with a 95-min orbital period in 47 Tucanae [47], one of 20 millisecond pulsars currently known in this cluster alone [81]. On-going surveys of clusters continue to yield new discoveries $[199,62]$.

\subsection{Going further}

Two excellent graduate-level monographs are available: the classic 1970s text Pulsars by Manchester \& Taylor [163] and the more up-to-date Pulsar Astronomy by Lyne \& Smith [156]. Those wishing to approach the subject from a more theoretical viewpoint are advised to read Michel's The Theory of Neutron Star Magnetospheres [167] and The Physics of the Pulsar Magnetosphere by Beskin, Gurevich \& Istomin [30]. Our summary of evolutionary aspects serves merely as a primer to the vast body of literature available. The reader is referred to the excellent review by Bhattacharya \& van den Heuvel [31] for further insights. For an excellent overview of pulsar distance measurements and their implications, see the review by Weisberg [263].

Living Reviews in Relativity (2001-5)

http://www.livingreviews.org 
Pulsar resources available on the Internet are continually becoming more extensive and useful. Good starting points for pulsar-surfers are the pages maintained at Arecibo [173], Berkeley [10], Bonn [165], Jodrell Bank [105], Princeton [196], Swinburne [230] and Sydney [197].

Living Reviews in Relativity (2001-5) http://www.livingreviews.org 


\section{The Galactic Pulsar Population}

Soon after the discovery of pulsars, it was realised that the observed sample is heavily biased towards the brighter objects that are the easiest to detect. What we observe therefore most likely represents only the tip of the iceberg of a much larger underlying population [88]. The extent to which the sample is incomplete is well demonstrated by the projection of pulsars onto the Galactic plane and their cumulative number distribution as a function of distance shown in Fig. 12. Although the clustering of sources around the Sun seen in the left panel of Fig. 12 would be consistent with Ptolemy's geocentric picture of the heavens, it is clearly at variance with what we now know about the Galaxy, where the massive stars show a radial distribution about the Galactic center.

The extent to which the pulsar sample is incomplete is shown in the right panel of Fig. 12 where the cumulative number of pulsars is plotted as a function of the projected distance from the Sun. The observed distribution is compared to the expected distribution for a simple model population in which there are errors in the distance scale, but no selection effects. We see that the observed sample becomes strongly deficient in terms of the number of sources for distances beyond a few kpc. We now discuss the main selection effects that hamper the detection of pulsars in some detail.

\subsection{Selection effects in pulsar searches}

\subsubsection{The inverse square law and survey thresholds}

The most prominent selection effect at play in the observed pulsar sample is the inverse square law, i.e. for a given intrinsic luminosity ${ }^{2}$, the observed flux density falls off as the inverse square of the distance. This results in the observed sample being dominated by nearby and/or bright objects. Beyond distances of a few kpc from the Sun, the apparent flux density falls below the flux thresholds $S_{\min }$ of most surveys. Following [71], we write:

$$
\begin{aligned}
S_{\text {min }}= & \operatorname{SNR}_{\text {min }}\left(\frac{T_{\text {rec }}+T_{\text {sky }}}{\mathrm{K}}\right)\left(\frac{G}{\mathrm{~K} \mathrm{Jy}^{-1}}\right)^{-1}\left(\frac{\Delta \nu}{\mathrm{MHz}}\right)^{-1 / 2}\left(\frac{t_{\text {int }}}{\mathrm{s}}\right)^{-1 / 2} . \\
& \left(\frac{W}{P-W}\right)^{1 / 2} \text { mJy, }
\end{aligned}
$$

where $\mathrm{SNR}_{\text {min }}$ is the threshold signal-to-noise ratio, $T_{\text {rec }}$ and $T_{\text {sky }}$ are the receiver and sky noise temperatures, $G$ is the gain of the antenna, $\Delta \nu$ is the observing bandwidth, $t_{\text {int }}$ is the integration time, $W$ is the detected pulse width and $P$ is the pulse period.

\footnotetext{
${ }^{2}$ Pulsar astronomers usually define the luminosity $L=S d^{2}$, where $S$ is the mean flux density at $400 \mathrm{MHz}$ (a standard observing frequency) and $d$ is the distance derived from the DM $(\S 2.3)$.
}

Living Reviews in Relativity (2001-5)

http://www.livingreviews.org 

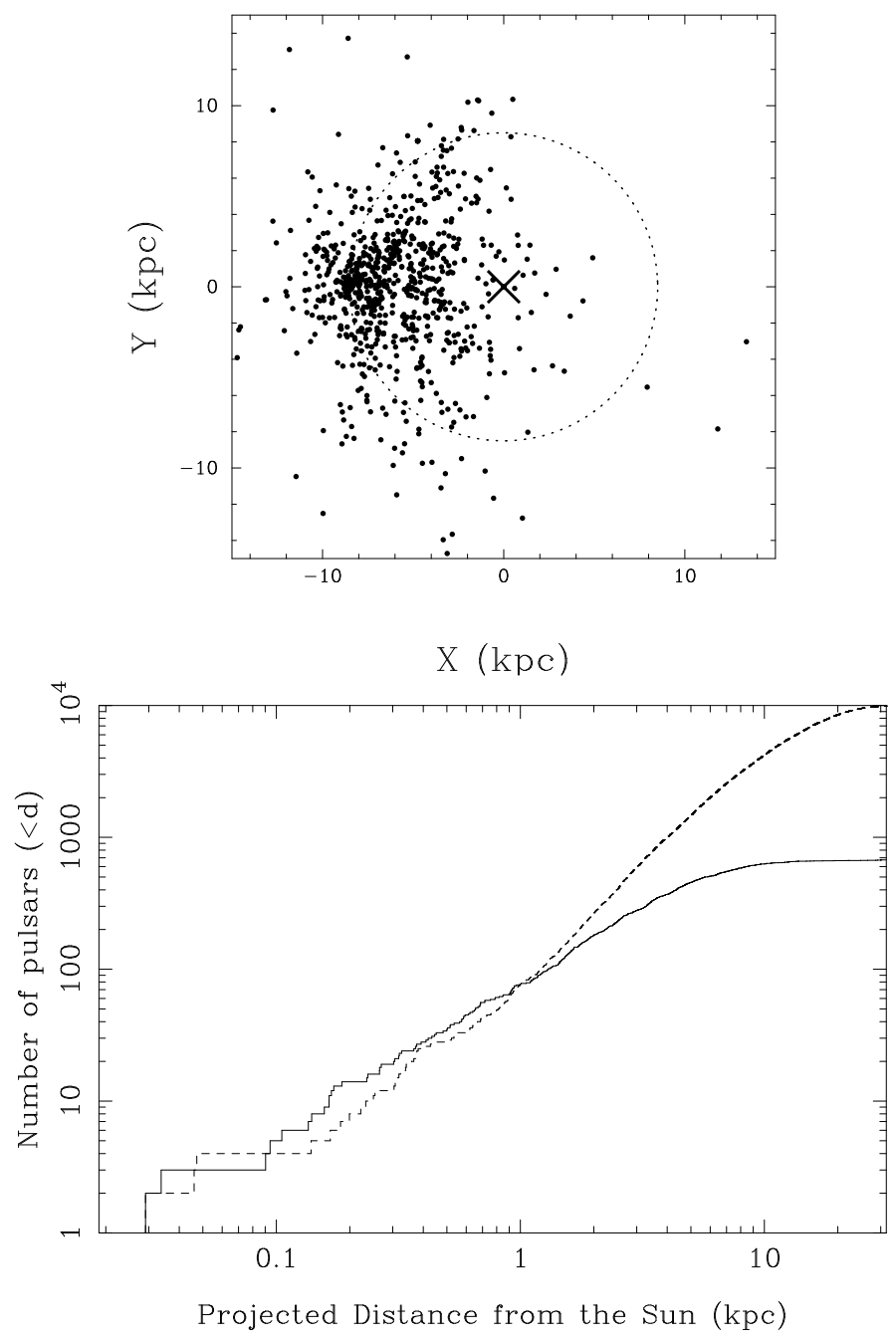

Figure 12: Left: The sample of radio pulsars from the Princeton catalog [196] projected onto the Galactic plane. The Galactic center is at $(0,0)$ and the Sun is at $(-8.5,0)$. Right: Cumulative number of observed pulsars (solid line) as a function of projected distance, $d$. The dashed line shows the expected distribution for a model population (see text). 


\subsubsection{Pulse dispersion and scattering}

It follows from Equation (3) that the sensitivity decreases as $W /(P-W)$ increases. Also note that if $W \gtrsim P$, the pulsed signal is smeared into the background emission and is no longer detectable, regardless of how luminous the source may be. The detected pulse width $W$ will be broader than the intrinsic value largely as a result of pulse dispersion and scattering by free electrons in the interstellar medium. As discussed above, the dispersive smearing scales as $\Delta \nu / \nu^{3}$, where $\nu$ is the observing frequency. This can largely be removed by dividing the pass-band into a number of channels and applying successively longer time delays to higher frequency channels before summing over all channels to produce a sharp profile. This process is known as incoherent dedispersion.

The smearing across the individual frequency channels, however, still remains and becomes significant at high dispersions when searching for shortperiod pulsars. Multi-path scattering results in a one-sided broadening due to the delay in arrival times which scales roughly as $\nu^{-4}$, which can not be removed by instrumental means. A simple scattering model is shown in Fig. 13 in which the scattering electrons are assumed to lie in a thin screen between the pulsar and the observer [210].

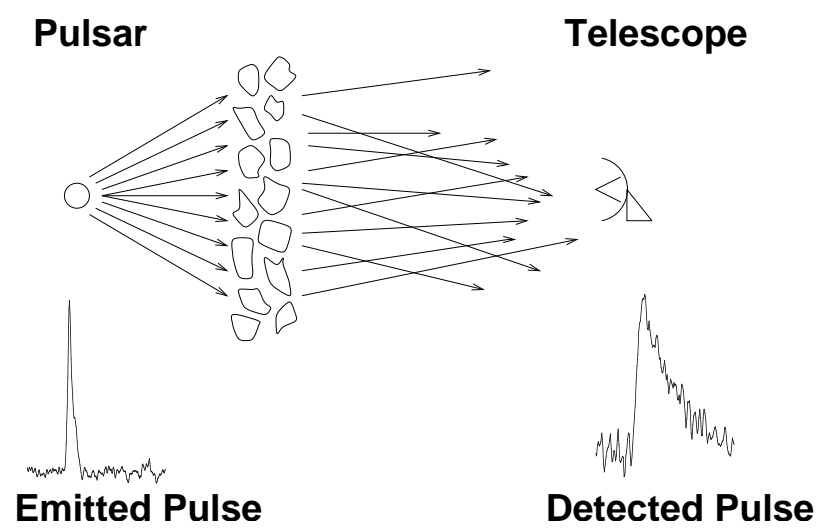

Figure 13: Pulse scattering caused by irregularities in the interstellar medium. The difference in path lengths and therefore in arrival times of the scattered rays results in a "scattering tail" in the observed pulse profile which lowers its signal-to-noise ratio.

Dispersion and scattering are most severe for distant pulsars in the inner Galaxy where the number of free electrons along the line of sight becomes large. The strong frequency dependence of both effects means that they are considerably less of a problem for surveys at observing frequencies $\gtrsim 1400 \mathrm{MHz}$ [56, 109] compared to the usual $400 \mathrm{MHz}$ search frequency. An added bonus for such observations is the reduction in $T_{\text {sky }}$, since the spectral index of the non-thermal Galactic emission is about -2.8 [126]. Pulsars themselves have steep radio spectra. Typical spectral indices are -1.6 [136], so that flux densities are roughly

Living Reviews in Relativity (2001-5)

http://www.livingreviews.org 
an order of magnitude lower at $1400 \mathrm{MHz}$ compared to $400 \mathrm{MHz}$. Fortunately, this can usually be compensated for by the use of larger receiver bandwidths at higher radio frequencies. For example, the $1370-\mathrm{MHz}$ system at Parkes has a bandwidth of $288 \mathrm{MHz}$ [147] compared to the 430-MHz system, where $32 \mathrm{MHz}$ is available [160].

\subsubsection{Orbital acceleration}

Standard pulsar searches use Fourier techniques to search for a-priori unknown periodic signals and usually assume that the apparent pulse period remains constant throughout the observation. For searches with integration times much greater than a few minutes this assumption is only valid for solitary pulsars, or those in binary systems where the orbital periods are longer than about a day. For shorter-period binary systems, as noted by Johnston \& Kulkarni [106], the Doppler-shifting of the period results in a spreading of the signal power over a number of frequency bins in the Fourier domain, leading to a reduction in signal-to-noise ratio. An observer will perceive the frequency of a pulsar to shift by an amount $a T /(P c)$, where $a$ is the (assumed constant) line-of-sight acceleration during the observation of length $T, P$ is the (constant) pulse period in its rest frame and $c$ is the speed of light. Given that the width of a frequency bin is $1 / T$, we see that the signal will drift into more than one spectral bin if $a T^{2} /(P c)>1$. Survey sensitivities to rapidly-spinning pulsars in tight orbits are therefore significantly compromised when the integration times are large.

As an example of this effect, as seen in the time domain, Fig. 14 shows a 22.5-min search mode observation of Hulse \& Taylor's famous binary pulsar B1913+16 [102, 239, 240]. Although this observation covers only about 5\% of the orbit $(7.75 \mathrm{hr})$, the effects of the Doppler smearing on the pulse signal are very apparent. While the standard search code (seeking constant periodicity) nominally detects the pulsar with a signal-to-noise ratio of 9.5 for this observation, it is clear that the Doppler shifting of the pulse period seen in the individual sub-integrations results in a significant reduction in signal-to-noise.

It is clearly desirable to employ a technique to recover the loss in sensitivity due to Doppler smearing. One such technique, the so-called "acceleration search" [168], assumes the pulsar has a constant acceleration during the inte-

gration. Each time series can then be re-sampled to refer it to the frame of an inertial observer using the Doppler formula to relate a time interval $\tau$ in the pulsar frame to that in the observed frame at time $t$, as $\tau(t) \propto(1+a t / c)$. Searching over a range of accelerations is desirable to find the time series for which the trial acceleration most closely matches the true value. In the ideal case, a time series is produced with a signal of constant period for which full sensitivity is recovered (see right panel of Fig. 14). Anderson et al. [5] used this technique to find PSR B2127+11C, a double neutron star binary in M15 which has parameters similar to B1913+16. Camilo et al. [47] have recently applied the same technique to 47 Tucanae to discover 9 binary pulsars, including one in a 96 -min orbit around a low-mass $\left(0.15 M_{\odot}\right)$ companion. This is currently the shortest binary period for any known radio pulsar.

Living Reviews in Relativity (2001-5)

http://www.livingreviews.org 


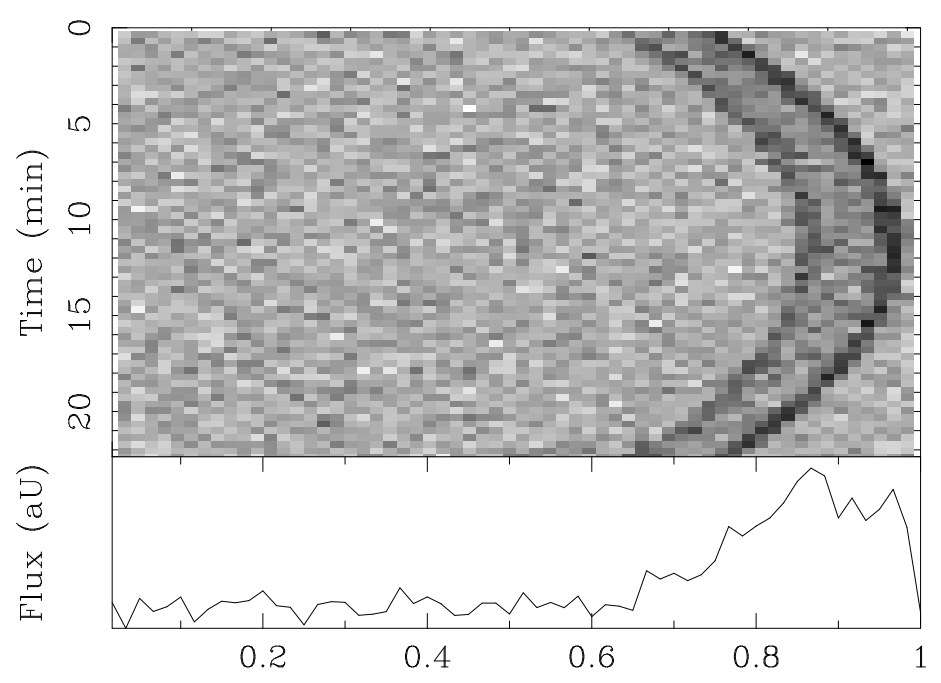

Pulse phase (periods)

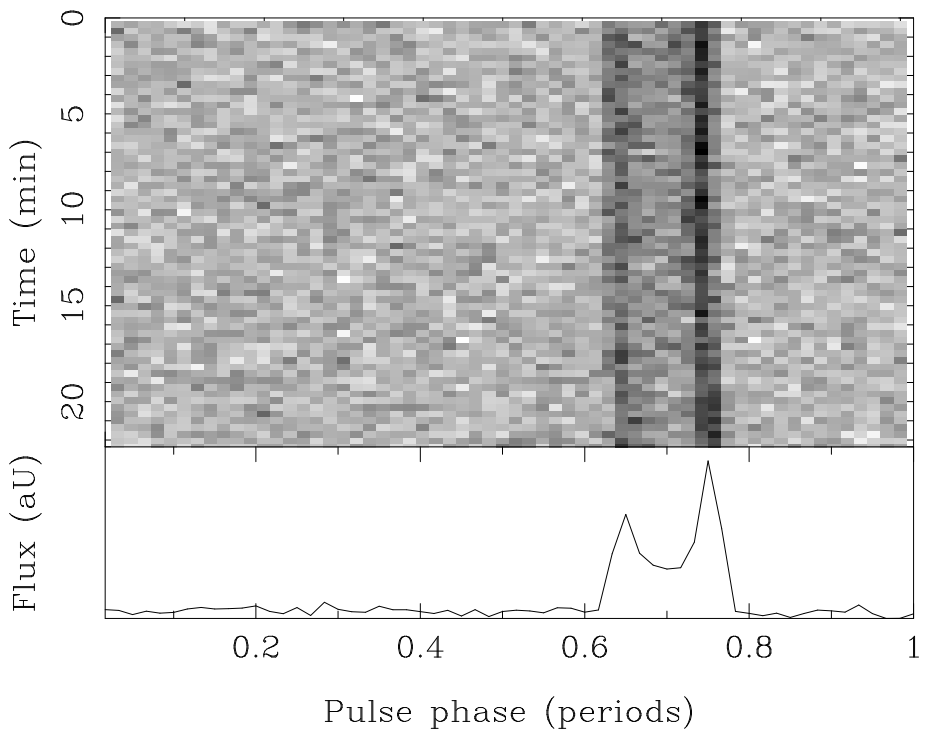

Figure 14: Left: A 22.5-min Arecibo observation of the binary pulsar B1913+16. The assumption that the pulsar has a constant period during this time is clearly inappropriate given the drifting in phase of the pulse during the integration (grey scale plot). Right: The same observation after applying an acceleration search. This shows the effective recovery of the pulse shape and a significant improvement in the signal-to-noise ratio.

Living Reviews in Relativity (2001-5)

http://www.livingreviews.org 
For the shortest orbital periods, the assumption of a constant acceleration during the observation clearly breaks down. Ransom et al. [199] have developed a particularly efficient algorithm for finding binaries whose orbits are so short that many orbits can take place during an integration. This phase modulation technique exploits the fact that the pulses are modulated by the orbit to create a family of periodic sidebands around the nominal spin period of the pulsar. This technique has already been used to discover a 1.7-hr binary pulsar in NGC 6544 [199]. The existence of these short-period radio pulsar binaries, as well as the 11-min X-ray binary X1820-303 in NGC 6624 [226], implies that there must be many more short-period binaries containing radio or X-ray pulsars in globular clusters that are waiting to be discovered by more sensitive searches.

\subsection{Correcting the observed pulsar sample}

\subsubsection{Scale factor determination}

Now that we have a flavour for the variety and severity of the selection effects that plague the observed sample of pulsars, how do we decouple these effects to form a less biased picture of the true population of objects? A very useful technique, first employed by Phinney \& Blandford and Vivekanand \& Narayan [191, 259], is to define a scaling factor $\xi$ as the ratio of the total Galactic volume weighted by pulsar density to the volume in which a pulsar could be detected by the surveys:

$$
\xi(P, L)=\frac{\iint_{\text {Galaxy }} \Sigma(R, z) R d R d z}{\iint_{P, L} \Sigma(R, z) R d R d z} .
$$

In this expression, $\Sigma(R, z)$ is the assumed pulsar distribution in terms of galactocentric radius $R$ and height above the Galactic plane $z$. Note that $\xi$ is primarily a function of period $P$ and luminosity $L$ such that short period/low-luminosity pulsars have smaller detectable volumes and therefore higher $\xi$ values than their long period/high-luminosity counterparts. This approach is similar to the classic $V / V_{\max }$ technique first used to correct observationally-biased samples of quasars [211].

This technique can be used to estimate the total number of active pulsars in the Galaxy. In practice, this is achieved by calculating $\xi$ for each pulsar separately using a Monte Carlo simulation to model the volume of the Galaxy probed by the major surveys [170]. For a sample of $N_{\text {obs }}$ observed pulsars above a minimum luminosity $L_{\min }$, the total number of pulsars in the Galaxy with luminosities above this value is simply

$$
\sum_{i=1}^{N_{\mathrm{obs}}} \frac{\xi_{i}}{f_{i}}
$$

where $f$ is the model-dependent "beaming fraction" discussed below in $\S 3.2 .3$. Monte Carlo simulations of the pulsar population incorporating the aforementioned selection effects have shown this method to be reliable, as long as $N_{\text {obs }}$ is reasonably large [131].

Living Reviews in Relativity (2001-5) http://www.livingreviews.org 


\subsubsection{The small-number bias}

For small samples of observationally-selected objects, the detected sources are likely to be those with larger-than-average luminosities. The sum of the scale factors (Equation (5)), therefore, will tend to underestimate the true size of the population. This "small-number bias" was first pointed out by Kalogera et al. $[112,113]$ for the sample of double neutron star binaries where we know of only three clear-cut examples (§ 3.4.1). Only when the number of sources in the sample gets past 10 or so does the sum of the scale factors become a good indicator of the true population size.

Model Galactic Population
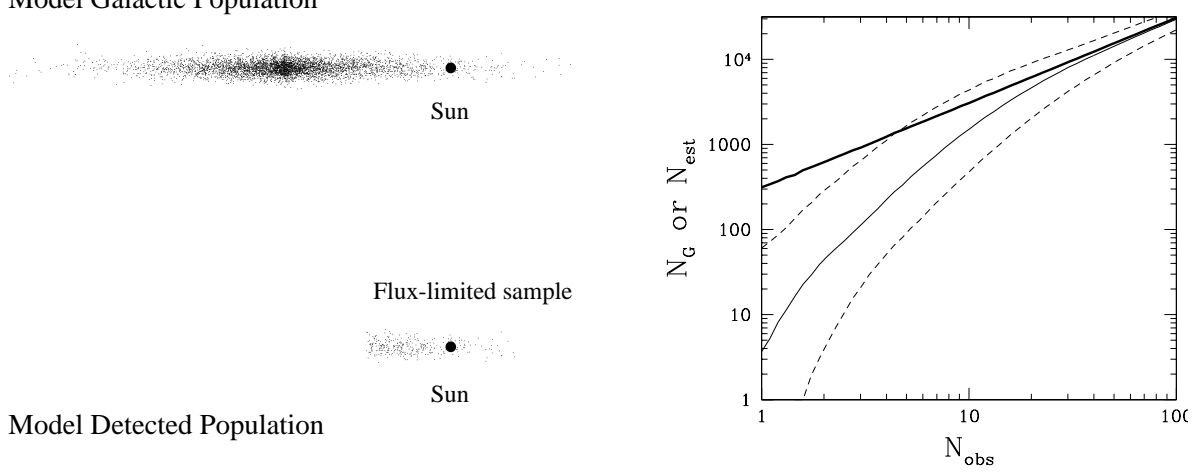

Figure 15: Small-number bias of the scale factor estimates derived from a synthetic population of sources where the true number of sources is known. Left: An edge-on view of a model Galactic source population. Right: The thick line shows $N_{\mathrm{G}}$, the true number of objects in the model Galaxy, plotted against $N_{\mathrm{obs}}$, the number detected by a flux-limited survey. The thin solid line shows $N_{\mathrm{est}}$, the median sum of the scale factors, as a function of $N_{\text {obs }}$ from a large number of Monte-Carlo trials. Dashed lines show 25 and $75 \%$ percentiles of the $N_{\text {est }}$ distribution.

\subsubsection{The beaming correction}

The "beaming fraction" $f$ in Equation (5) is simply the fraction of $4 \pi$ steradians swept out by the radio beam during one rotation. Thus $f$ gives the probability that the beam cuts the line-of-sight of an arbitrarily positioned observer. A naïve estimate of $f$ is $20 \%$; this assumes a beam width of $\sim 10^{\circ}$ and a randomly distributed inclination angle between the spin and magnetic axes [238]. Observational evidence suggests that shorter period pulsars have wider beams and therefore larger beaming fractions than their long-period counterparts $[171,149,32,231]$. It must be said, however, that a consensus on the beaming fraction-period relation has yet to be reached. This is shown in Fig. 16 where we compare the period dependence of $f$ as given by a number of models. Adopting the Lyne \& Manchester model, pulsars with periods $\sim 100 \mathrm{~ms}$ beam

Living Reviews in Relativity (2001-5)

http://www. livingreviews.org 
to about $30 \%$ of the sky compared to the Narayan \& Vivekanand model in which pulsars with periods below $100 \mathrm{~ms}$ beam to the entire sky.

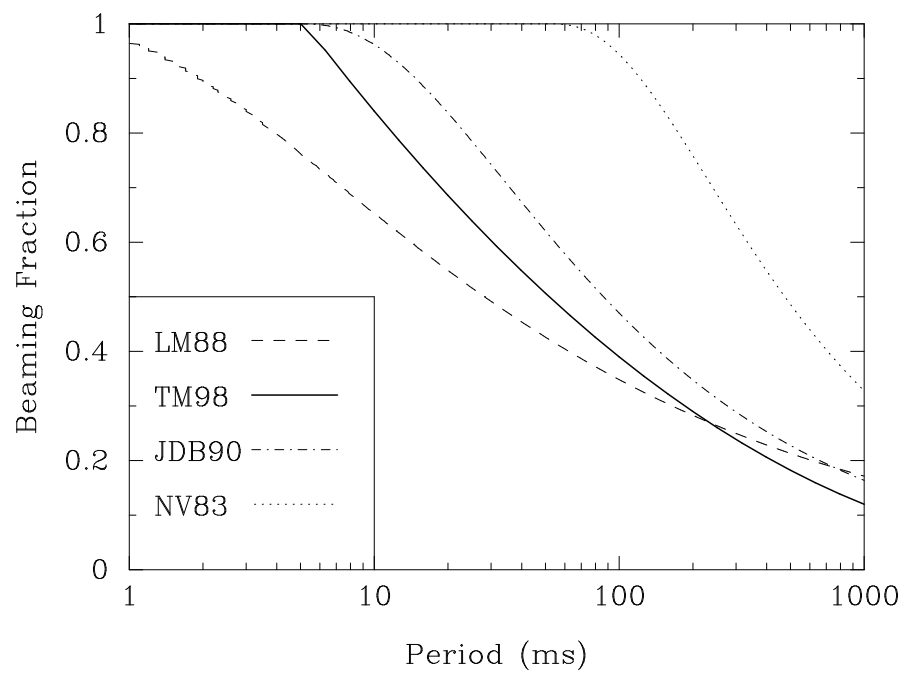

Figure 16: Beaming fraction plotted against pulse period for four different beaming models: Tauris \& Manchester 1998 (TM88; [231]), Lyne \& Manchester 1988 (LM88; [149]), Biggs 1990 (JDB90; [32]) and Narayan \& Vivekanand 1983 (NV83; [171]).

When most of these beaming models were originally proposed, the sample of millisecond pulsars was $\lesssim 5$ and hence their predictions about the beaming fractions of short-period pulsars relied largely on extrapolations from the normal pulsars. A recent analysis of a large sample of millisecond pulsar profiles by Kramer et al. [122] suggests that the beaming fraction of millisecond pulsars lies between 50 and $100 \%$.

\subsection{The population of normal and millisecond pulsars}

\subsubsection{Luminosity distributions and local number estimates}

The most recent use of the scale factor approach to derive the characteristics of the true normal and millisecond pulsar populations is based on the sample of pulsars within $1.5 \mathrm{kpc}$ of the Sun [152]. The rationale for this cut-off is that, within this region, the selection effects are well understood and easier to quantify by comparison with the rest of the Galaxy. These calculations should give reliable estimates for the local pulsar population.

The luminosity distributions obtained from this analysis are shown in Fig. 17. For the normal pulsars, integrating the corrected distribution above $1 \mathrm{mJy} \mathrm{kpc}{ }^{2}$ and dividing by $\pi \times(1.5)^{2} \mathrm{kpc}^{2}$ yields a local surface density, assuming Biggs' beaming model [32] of $156 \pm 31$ pulsars $\mathrm{kpc}^{-2}$. The same analysis for the mil- 

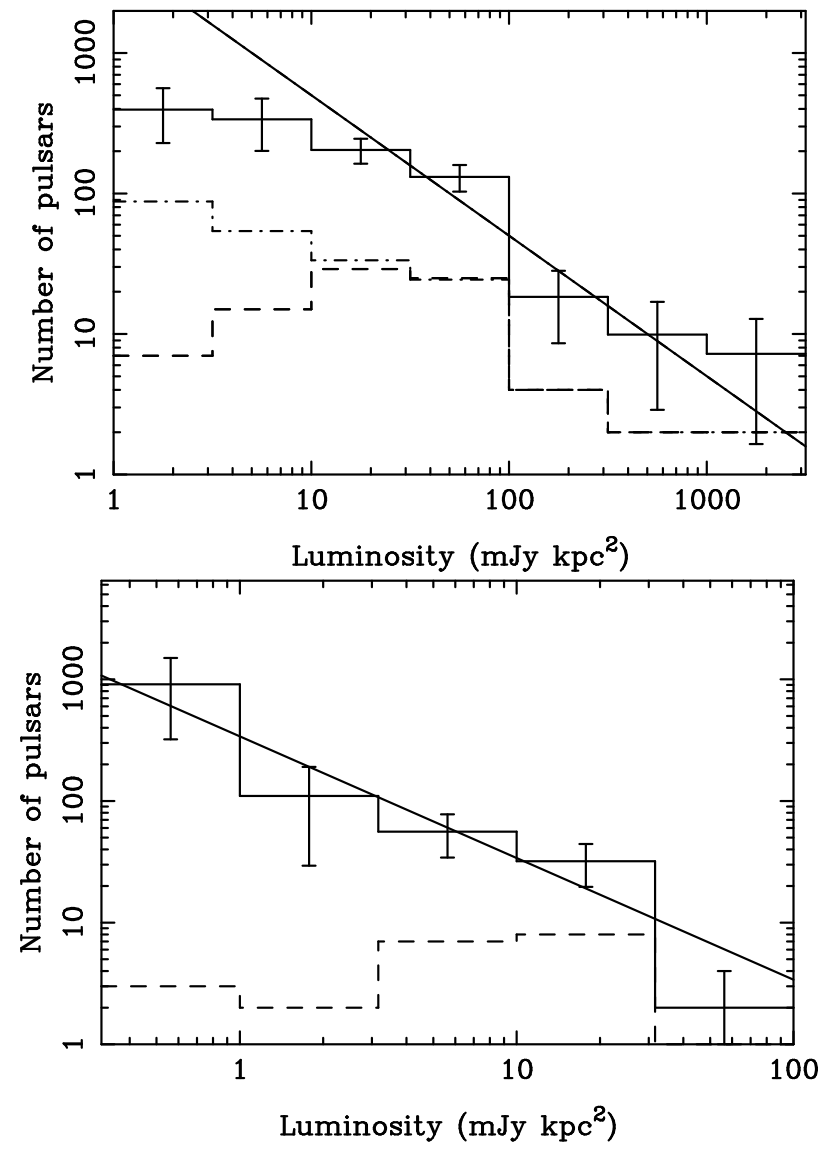

Figure 17: Left: The corrected luminosity distribution (solid histogram with error bars) for normal pulsars. The corrected distribution before the beaming model has been applied is shown by the dot-dashed line. Right: The corresponding distribution for millisecond pulsars. In both cases, the observed distribution is shown by the dashed line and the thick solid line is a power law with a slope of -1 . The difference between the observed and corrected distributions highlights the severe under-sampling of low-luminosity pulsars.

Living Reviews in Relativity (2001-5)

http://www.livingreviews.org 
lisecond pulsars, assuming a mean beaming fraction of $75 \%$ [122], leads to a local surface density of $38 \pm 16$ pulsars $\mathrm{kpc}^{-2}$ for luminosities above $1 \mathrm{mJy} \mathrm{kpc}$.

\subsubsection{Galactic population and birth-rates}

Integrating the local surface densities of pulsars over the whole Galaxy requires a knowledge of the presently rather uncertain Galactocentric radial distribution $[153,107]$. One approach is to assume that pulsars have a radial distribution similar to that of other stellar populations and scale the local number density with this distribution to estimate the total Galactic population. The corresponding local-to-Galactic scaling is $1000 \pm 250 \mathrm{kpc}^{2}$ [200]. With this approach we estimate there to be $\sim 160,000$ active normal pulsars and $\sim 40,000$ millisecond pulsars in the Galaxy. Based on these estimates, we are in a position to deduce the corresponding rate of formation or birth-rate. From the $P-\dot{P}$ diagram in Fig. 7 , we infer a typical lifetime for normal pulsars of $\sim 10^{7} \mathrm{yr}$, corresponding to a Galactic birth rate of $\sim 1$ per $60 \mathrm{yr}$ - consistent with the rate of supernovae [253]. As noted in $\S 2.4$, the millisecond pulsars are much older, with ages close to that of the Universe $\tau_{\mathrm{u}}$ (we assume here $\tau_{\mathrm{u}}=10 \mathrm{Gyr}$ [104]). Taking the maximum age of the millisecond pulsars to be $\tau_{\mathrm{u}}$, we infer a mean birth rate of at least $4 \times 10^{-6} \mathrm{yr}^{-1}$. This is consistent, within the uncertainties, with the birth-rate of low-mass X-ray binaries [135].

\subsubsection{Implications for gravitational wave detectors}

The estimates of the local surface density of active pulsars allow us to deduce the likely distance of the nearest neutron star to Earth. For the combined millisecond and normal pulsar populations, with a surface density of $193 \pm 35$ pulsars $\mathrm{kpc}^{-2}$, the nearest neutron star is thus likely to be $\lesssim 40 \mathrm{pc}$. This number is of interest to those building gravitational wave detectors, since it determines the likely amplitude of gravitational waves emitted from nearby rotating neutron stars [213]. According to Thorne [244], currently planned detectors will be able to detect neutron stars with ellipticities greater than $7.5 \times 10^{-11}(P d)^{2}$, where $P$ is the rotation period in ms and $d$ is the distance in kpc. The recent probable detection of free precession in the radio pulsar B1828-11 [221] does indicate that ellipticities exist in neutron stars so that nearby objects may be continuous sources of gravitational radiation.

Thus, in order to detect small ellipticities, nearby sources with short spin periods are required. One of the best candidates is the nearby 5.75 -ms pulsar J0437-4715 [108]. At a distance of $178 \pm 26 \mathrm{pc}$ [208] this is currently the closest known millisecond pulsar to the Earth. The closest known neutron star is RX J185635-3754 discovered in the ROSAT all-sky survey [261]. Multi-epoch HST observations show that this isolated neutron star is located at a distance of $61 \pm 9 \mathrm{pc}$ [260]. In keeping with other radio-quiet isolated neutron stars, the period of this pulsar is likely to be several seconds [176].

Living Reviews in Relativity (2001-5)

http://www.livingreviews.org 


\subsection{The population of relativistic binaries}

Of particular interest to the astronomical community at large are the numbers of relativistic binary systems in the Galaxy. Systems involving neutron stars are: double neutron star binaries, neutron star-white dwarf binaries and neutron star-black hole binaries. Interest in these systems is two-fold: (1) to test the predictions of general relativity against alternative theories of strong-field gravity using the radio pulsar as a highly stable clock moving in the strong gravitational field; (2) to detect strong gravitational wave emission from coalescing binaries with upcoming gravitational-wave observatories like GEO600 and LIGO, VIRGO and TAMA [244, 212]. For a Living Reviews article on this new generation of interferometric gravitational wave detectors see [100].

Although no radio pulsar in orbit around a black hole companion has so far been observed, we now know of several double neutron star and neutron-star white dwarf binaries which will merge due to gravitational wave emission within a reasonable time-scale. The merging time $\tau_{\mathrm{g}}$ of a binary system containing two compact objects due to the emission of gravitational radiation can be calculated from the following formula which requires only the component masses and current orbital period $P_{\mathrm{b}}$ and eccentricity $e$ :

$$
\tau_{\mathrm{g}} \simeq 10^{7} \mathrm{yr}\left(\frac{P_{\mathrm{b}}}{\mathrm{hr}}\right)^{8 / 3}\left(\frac{m_{1}+m_{2}}{M}\right)^{1 / 3}\left(\frac{\mu}{M}\right)_{\odot}^{-1}\left(1-e^{2}\right)^{7 / 2} .
$$

Here $m_{1,2}$ are the masses of the two stars and $\mu=m_{1} m_{2} /\left(m_{1}+m_{2}\right)$. This formula is a good analytic approximation (within a few percent) to the numerical solution of the exact equations for $\tau_{\mathrm{g}}$ in the original papers by Peters \& Mathews [187, 188]. In the following subsections we review current knowledge on the population sizes and merging rates of such binaries where one component is visible as a radio pulsar.

\subsubsection{Double neutron star binaries}

As noted in $\S 2.4$, double neutron star (DNS) binaries are expected to be rare since the binary system has to survive two supernova explosions. This expectation is certainly borne out by radio pulsar searches which have revealed only three certain DNS binaries so far: PSRs B1534+12 [270], B1913+16 [102] and B2127+11C [195]. Although we cannot see the companion neutron star in any of these systems, we are "certain" of the identification from the precise measurements of the component masses via relativistic effects measured in pulsar timing observations (see $\S 4.1$ ). The spin and orbital parameters of these pulsars are listed in Table 1. Also listed in Table 1 are three further DNS candidates with eccentric orbits and large mass functions but for which there is presently not sufficient component mass information to confirm their nature.

Despite the uncertainties in identifying DNS binaries, for the purposes of determining the Galactic merger rate, the systems for which $\tau_{\mathrm{g}}$ is less than $\tau_{\mathrm{u}}$ (i.e. PSRs B1534+12, B1913+16 and B2127+11C) are primarily of interest. Of these PSR B2127+11C is in the process of being ejected from the globular

Living Reviews in Relativity (2001-5)

http://www.livingreviews.org 


\begin{tabular}{lrrr}
\hline \hline & $\mathrm{J} 1518+4904$ & $\mathrm{~B} 1534+12$ & $\mathrm{~J} 1811-1736$ \\
\hline$P[\mathrm{~ms}]$ & 40.9 & 37.9 & 104.2 \\
$P_{\mathrm{b}}[\mathrm{d}]$ & 8.6 & 0.4 & 18.8 \\
$e$ & 0.25 & 0.27 & 0.83 \\
$\tau_{\mathrm{c}}\left[10^{8} \mathrm{y}\right]$ & 200 & 2.5 & 970 \\
$\tau_{\mathrm{g}}\left[10^{8} \mathrm{y}\right]$ & $\gg \tau_{\mathrm{u}}$ & 27 & $\gg \tau_{\mathrm{u}}$ \\
Masses measured? & No & Yes & No \\
\hline \hline & $\mathrm{B} 1820-11$ & $\mathrm{~B} 1913+16$ & $\mathrm{~B} 2127+11 \mathrm{C}$ \\
\hline$P[\mathrm{~ms}]$ & 279.8 & 59.0 & 30.5 \\
$P_{\mathrm{b}}[\mathrm{d}]$ & 357.8 & 0.3 & 0.3 \\
$e$ & 0.79 & 0.62 & 0.68 \\
$\tau_{\mathrm{c}}\left[10^{8} \mathrm{y}\right]$ & 0.04 & 1.1 & 0.97 \\
$\tau_{\mathrm{g}}\left[10^{8} \mathrm{y}\right]$ & $\gg \tau_{\mathrm{u}}$ & 3.0 & 2.2 \\
Masses measured? & No & Yes & Yes \\
\hline & & &
\end{tabular}

Table 1: Known DNS binaries and candidates. Listed are the pulse period $P$, the orbital period $P_{\mathrm{b}}$, the orbital eccentricity $e$, the pulsar characteristic age $\tau_{\mathrm{c}}$, and the expected binary coalescence time-scale $\tau_{\mathrm{g}}$ due to gravitational wave emission calculated from Equation (6). Cases for which $\tau_{\mathrm{g}}$ is a factor of 100 or more greater than the age of the Universe are listed as $\gg \tau_{\mathrm{u}}$. To distinguish between definite and candidate DNS systems, we also list whether the masses of both components have been determined.

cluster M15 [195, 192] and is thought to make only a negligible contribution to the merger rate [190]. The general approach with the remaining two systems is to derive scale factors for each object (as outlined in $\S 3.2 .1$ ) and then divide these by a reasonable estimate for the lifetime. In what follows we summarize the main studies of this kind. The most comprehensive investigation of the DNS binary population to date is the recent study by Kalogera et al. (hereafter KNST; [113]).

As discussed in $\S 3.2 .1$, scale factors are dependent on the assumed pulsar distribution. The key parameter here is the scale height of the population with respect to the Galactic plane which itself is a function of the velocity distribution of the population. KNST examined this dependence in detail and found scale heights in the range $0.8-1.7 \mathrm{kpc}$. Based on this range, KNST revised earlier scale factor estimates [60] to 145-200 for B1534+12 and 45-60 for PSR B1913+16. As mentioned in $\S 3.2 .2$ scale factors calculated from a small sample of objects are subject to a significant bias. KNST find the bias in their sample to be anywhere between 2 and 200. This boosts the scale factors to the range 190-40000 for B1534+12 and 90-12000 for B1913+16.

The above scale factors also require a beaming correction. As noted in $\S 3.2 .3$, current radio pulsar beaming models vary considerably. Fortunately, for the two pulsars under consideration, detailed studies of the beam sizes [9, 119, 262] lead KNST to conclude that both pulsars beam to only about a sixth of the entire sky. 
The beaming-corrected numbers suggest a total of between 1680 and 312,000 active DNS binaries in our Galaxy. Many of these systems will be extremely faint objects. These estimates are dominated by the small-number bias factor. KNST's study highlights the importance of this effect.

Some debate exists about what is the most reasonable estimate of the lifetime. Phinney [190] defines this as the sum of the pulsar's spin-down age plus $\tau_{\mathrm{g}}$ defined above. A few years later, van den Heuvel and myself argued [255] that a more likely estimate can be obtained by appealing to steady-state arguments where we expect sources to be created at the same rate at which they are merging. The mean lifetime was then found to be about three times the current spin-down age. This argument does, however, depend on the luminosity evolution of radio pulsars which is currently only poorly understood. Arzoumanian, Cordes \& Wasserman [7] used kinematic data to constrain the most likely ages of the DNS binaries. They note that the remaining detectable lifetime should also take account of the reduced detectability at later epochs due to acceleration smearing as the DNS binary becomes more compact due to gravitational wave emission. KNST concluded that the lifetimes are dominated by the latter time-scale which, following Arzoumanian et al., they took to be the time for the orbital period to halve. The resulting lifetimes are $2.5 \times 10^{9} \mathrm{yr}$ for B1534+12 and $2.5 \times 10^{8}$ yr for B1913+16.

Taking these number and lifetime estimates, KNST find the Galactic merger rate of DNS binaries to range between $3 \times 10^{-6}$ and $4 \times 10^{-4} \mathrm{yr}^{-1}$. Extrapolating this number out to include DNS binaries detectable by LIGO in other galaxies á la Phinney [190], KNST find the expected event rate to be $<0.25 \mathrm{yr}^{-1}$ for LIGO-I and 2-1300 $\mathrm{yr}^{-1}$ for LIGO-II. Thus, despite the uncertainties, it seems that the prospects for detecting gravitational-wave emission from DNS inspirals in the near future are most promising.

\subsubsection{White dwarf-neutron star binaries}

The population of white dwarf-neutron star (WDNS) sources containing a young radio pulsar has only recently been confirmed by observers following the identification of a white dwarf companion to the binary pulsar B2303+46 by van Kerkwijk et al. [256]. Previously, this eccentric binary pulsar was thought to be an example of a DNS binary in which the visible pulsar is the second-born neutron star [229, 143]. The optical identification rules this out and now strongly suggests a scenario in which the white dwarf was formed first. In this case, material was transfered onto the secondary during the giant phase of the primary so that the secondary became massive enough to form a neutron star [256, 232].

PSR B2303+46 has a long orbital period and does not contribute significantly to the overall merger rate of WDNS binaries. The new discovery of PSR J1141-6545 [116], which will merge due to gravitational-wave emission within $1.3 \mathrm{Gyr}$, is suggestive of a large population of similar binaries. This is particularly compelling when one considers that the radio lifetime of the visible pulsar is only a fraction of total lifetime of the binary before coalescence due to gravitational-wave emission. Edwards \& Bailes [75] estimate there to be 850

Living Reviews in Relativity (2001-5)

http://www.livingreviews.org 
WDNS binaries within $3 \mathrm{kpc}$ of the Sun which will merge within $\tau_{\mathrm{u}}$.

Population syntheses by Tauris \& Sennels [232] suggest that the formation rate of WDNS binaries is between 10-20 times that of DNS binaries. Based on the merging rate estimates for DNS binaries discussed in the previous section, this translates to a merging rate of WDNS binaries of between $3 \times 10^{-5} \mathrm{yr}^{-1}$ and $8 \times 10^{-3} \mathrm{yr}^{-1}$. In summary, although statistics are necessarily poor at this stage, coalescing WDNS binaries look to be very promising sources for gravitational wave detectors.

\subsection{Going further}

Studies of pulsar population statistics represent a large proportion of the pulsar literature. During this section we have tried to cite many of the key papers in this field. Good starting points for further reading can be found in other review articles $[167,31,156]$. Our coverage of compact object coalescence rates has concentrated on empirical methods and we have hopefully convinced the reader that these are fair and straightforward.

An alternative approach is to undertake a full-blown Monte Carlo simulation of the most likely evolutionary scenarios described in $\S 2.4 .3$. In this "scenariomachine" approach, a population of primordial binaries is synthesized with a number of underlying distribution functions: primary mass, binary mass ratio, orbital period distribution etc. The evolution of both stars is then followed to give a predicted sample of binary systems of all the various types. Since the full range of binary parameters is known, the merger rates of each type of binary are then automatically predicted by this model without the need to debate what the likely coalescence times will be. Selection effects are not normally taken into account in this approach. The final census is usually normalized to the star formation rate. Numerous examples of the scenario-machine approach (most often to populations of binaries where one or both members are NSs) can be found in the literature [69, 204, 252]. These include the widely-cited code, developed by Lipunov and collaborators to perform population syntheses of binary stars [227]. Although extremely instructive, the uncertain assumptions about initial conditions, the physics of mass transfer and the kicks applied to the compact object at birth result in a wide range of predicted event rates which are currently broader than the empirical methods [112]. Ultimately, the detection statistics from the gravitational wave detectors could provide far tighter constraints on the DNS merging rate than the pulsar surveys from which these predictions are made.

An excellent overview of gravitational-wave astronomy and the detection of gravitational waves from inspiraling binaries is presented by Thorne in his presentation at the centennial meeting of the American Physical Society which is available on-line [243].

Living Reviews in Relativity (2001-5)

http://www.livingreviews.org 


\section{Pulsar Timing}

It became clear soon after their discovery that pulsars are excellent celestial clocks. In the original discovery paper [97], the period of the first pulsar to be discovered, PSR B1919+21, was found to be stable to one part in $10^{7}$ over a time-scale of a few months. Following the discovery of the millisecond pulsar B1937+21 in 1982 [17] it was demonstrated that its period could be measured to one part in $10^{13}$ or better [65]. This unrivaled stability leads to a host of applications including time keeping, probes of relativistic gravity and natural gravitational wave detectors.

\subsection{Observing basics}

As each new pulsar is discovered, the standard practice is to add it to a list of pulsars which are regularly observed at least once or twice per month by large radio telescopes throughout the world. The schematic diagram shown in Fig. 18 summarises the essential steps involved in such a "time-of-arrival" (TOA) measurement. Incoming pulses emitted by the rotating neutron star traverse the interstellar medium before being received by the radio telescope. After amplification by high sensitivity receivers, the pulses are de-dispersed and added to form a mean pulse profile.

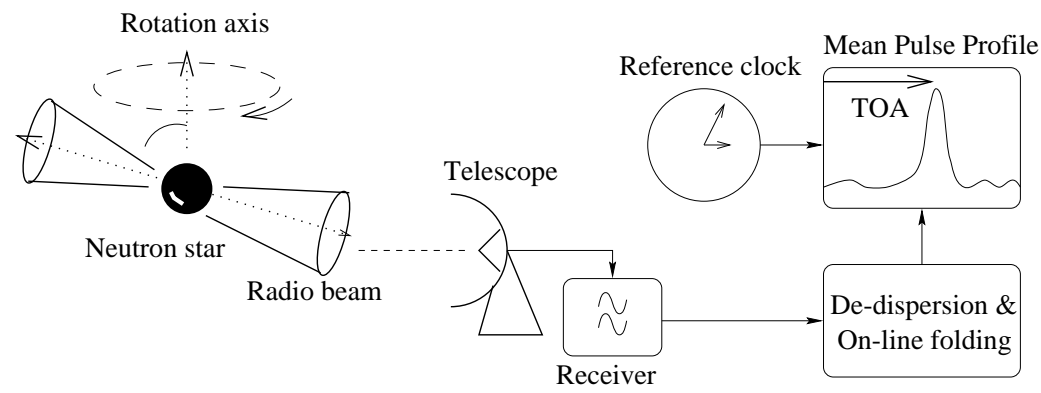

Figure 18: Schematic showing the main stages involved in pulsar timing observations.

During the observation, the data regularly receive a time stamp, usually based on a caesium time standard or hydrogen maser at the observatory plus a signal from the Global Positioning System of satellites (GPS; [64]). The TOA of this mean pulse is then defined as the arrival time of some fiducal point on the profile. Since the mean profile has a stable form at any given observing frequency $(\S 2.2)$, the TOA can be accurately determined by a simple crosscorrelation of the observed profile with a high signal-to-noise "template" profile obtained from the addition of many observations of the pulse profile at the particular observing frequency.

Success in pulsar timing hinges on how precisely the fiducial point can be determined. This is largely dependent on the signal-to-noise ratio (SNR) of

Living Reviews in Relativity (2001-5)

http://www.livingreviews.org 
the mean pulse profile. The uncertainty in a TOA measurement $\epsilon_{\mathrm{TOA}}$ is given roughly by the pulse width divided by the SNR. Using Equation (3), we can express this as a fraction of the pulse period:

$$
\frac{\epsilon_{\mathrm{TOA}}}{P} \simeq\left(\frac{T_{\mathrm{rec}}+T_{\mathrm{sky}}}{\mathrm{K}}\right)\left(\frac{G}{\mathrm{~K} \mathrm{Jy}^{-1}}\right)^{-1}\left(\frac{\Delta \nu}{\mathrm{MHz}}\right)^{-1 / 2}\left(\frac{t_{\mathrm{int}}}{\mathrm{s}}\right)^{-1 / 2}\left(\frac{W}{P}\right)^{3 / 2} .
$$

In this expression $T_{\text {rec }}$ and $T_{\text {sky }}$ are the receiver and sky noise temperatures, $G$ is the gain of the antenna, $\Delta \nu$ is the observing bandwidth, $t_{\text {int }}$ is the integration time, $W$ is the pulse width and $P$ is the pulse period (we assume $W \ll P$ ). Optimum results are thus obtained for observations of short period pulsars with large flux densities and narrow duty cycles $(W / P)$ using large telescopes with low-noise receivers and large observing bandwidths.

One of the main problems of employing large bandwidths is pulse dispersion. As discussed in $\S 2.3$, the velocity of the pulsed radiation through the ionised interstellar medium is frequency-dependent: pulses emitted at higher radio frequencies travel faster and arrive earlier than those emitted at lower frequencies. This process has the effect of "stretching" the pulse across a finite receiver bandwidth, reducing the apparent signal-to-noise ratio and therefore increasing $\epsilon_{\mathrm{TOA}}$. For most normal pulsars, this process can largely be compensated for by the incoherent de-dispersion process outlined in $\S 3.1$.

To exploit the precision offered by millisecond pulsars, a more precise method of dispersion removal is required. Technical difficulties in building devices with very narrow channel bandwidths require another dispersion removal technique. In the process of coherent de-dispersion [90] the incoming signals are de-dispersed over the whole bandwidth using a filter which has the inverse transfer function to that of the interstellar medium. The signal processing can be done either on-line using finite impulse response filter devices [14] or off-line in software [219, 222]. The on-line approach allows for large bandwidths to be employed and real-time viewing of the data. Off-line reduction, while slow and computationally expensive, allows for more flexible data reduction schemes as well as periodicity searches to be carried out.

The maximum time resolution obtainable via coherent dedispersion is the inverse of the receiver bandwidth. For bandwidths of $10 \mathrm{MHz}$, this technique makes it possible to resolve features on time-scales as short as $100 \mathrm{~ns}$. This corresponds to probing regions in the neutron star magnetosphere as small as $30 \mathrm{~m}$ !

\subsection{The timing model}

Ideally, in order to model the rotational behaviour of the neutron star, we require TOAs measured by an inertial observer. An observatory located on Earth experiences accelerations with respect to the neutron star due to the Earth's rotation and orbital motion around the Sun and is therefore not in an inertial frame. To a very good approximation, the centre-of-mass of the solar system, the solar system barycentre, can be regarded as an inertial frame. It 
is standard practice [103] to transform the observed TOAs to this frame using a planetary ephemeris such as the JPL DE200 [224]. The transformation is summarised as the difference between barycentric $(\mathcal{T})$ and observed $(t)$ TOAs:

$$
\mathcal{T}-t=\frac{\underline{r} \cdot \underline{\hat{s}}}{c}+\frac{(\underline{r} \cdot \underline{\hat{s}})^{2}-|\underline{r}|^{2}}{2 c d}+\Delta t_{\mathrm{rel}}-\Delta t_{\mathrm{DM}}
$$

Here $\underline{r}$ is the position of the Earth with respect to the barycentre, $\underline{\hat{s}}$ is a unit vector in the direction towards the pulsar at a distance $d$, and $c$ is the speed of light. The first term on the right hand side of this expression is the light travel time from the Earth to the solar system barycentre. For all but the nearest pulsars, the incoming pulses can be approximated by plane wavefronts. The second term, which represents the delay due to spherical wavefronts and which yields the trigonometric parallax and hence $d$, is presently only measurable for four nearby millisecond pulsars [117, 46, 208]. The term $\Delta t_{\text {rel }}$ represents the Einstein and Shapiro corrections due to general relativistic effects within the solar system [16]. Since measurements are often carried out at different observing frequencies with different dispersive delays, the TOAs are generally referred to the equivalent time that would be observed at infinite frequency. This transformation corresponds to the term $\Delta t_{\mathrm{DM}}$ and may be calculated from Equation (1).

Following the accumulation of about ten to twenty barycentric TOAs from observations spaced over at least several months, a surprisingly simple model can be applied to the TOAs and optimised so that it is sufficient to account for the arrival time of any pulse emitted during the time span of the observations and predict the arrival times of subsequent pulses. The model is based on a Taylor expansion of the angular rotational frequency $\Omega=2 \pi / P$ about a model value $\Omega_{\circ}$ at some reference epoch $t_{\circ}$. The model pulse phase $\phi$ as a function of barycentric time is thus given by:

$$
\phi(\mathcal{T})=\phi_{\circ}+\left(\mathcal{T}-\mathcal{T}_{\circ}\right) \Omega_{\circ}+\frac{1}{2}\left(\mathcal{T}-\mathcal{T}_{\circ}\right)^{2} \dot{\Omega}_{\circ}+\cdots,
$$

where $\phi_{\circ}$ is the pulse phase at $\mathcal{T}_{\circ}$. Based on this simple model, and using initial estimates of the position, dispersion measure and pulse period, a "timing residual" is calculated for each TOA as the difference between the observed and predicted pulse phases.

A set of timing residuals for the nearby pulsar B1133+16 spanning almost 10 years is shown for illustrative purposes in Fig. 19. Ideally, the residuals should have a zero mean and be free from any systematic trends (Fig. 19a). Inevitably, however, due to our a-priori ignorance of the rotational parameters, the model needs to be refined in a bootstrap fashion. Early sets of residuals will exhibit a number of trends indicating a systematic error in one or more of the model parameters, or a parameter not initially incorporated into the model.

From Equation (9), an error in the assumed $\Omega_{\circ}$ results in a linear slope with time. A parabolic trend results from an error in $\dot{\Omega}_{\circ}$ (Fig. 19b). Additional effects will arise if the assumed position of the pulsar (the unit vector $\underline{\hat{s}}$ in equation (8))

Living Reviews in Relativity (2001-5)

http://www.livingreviews.org 

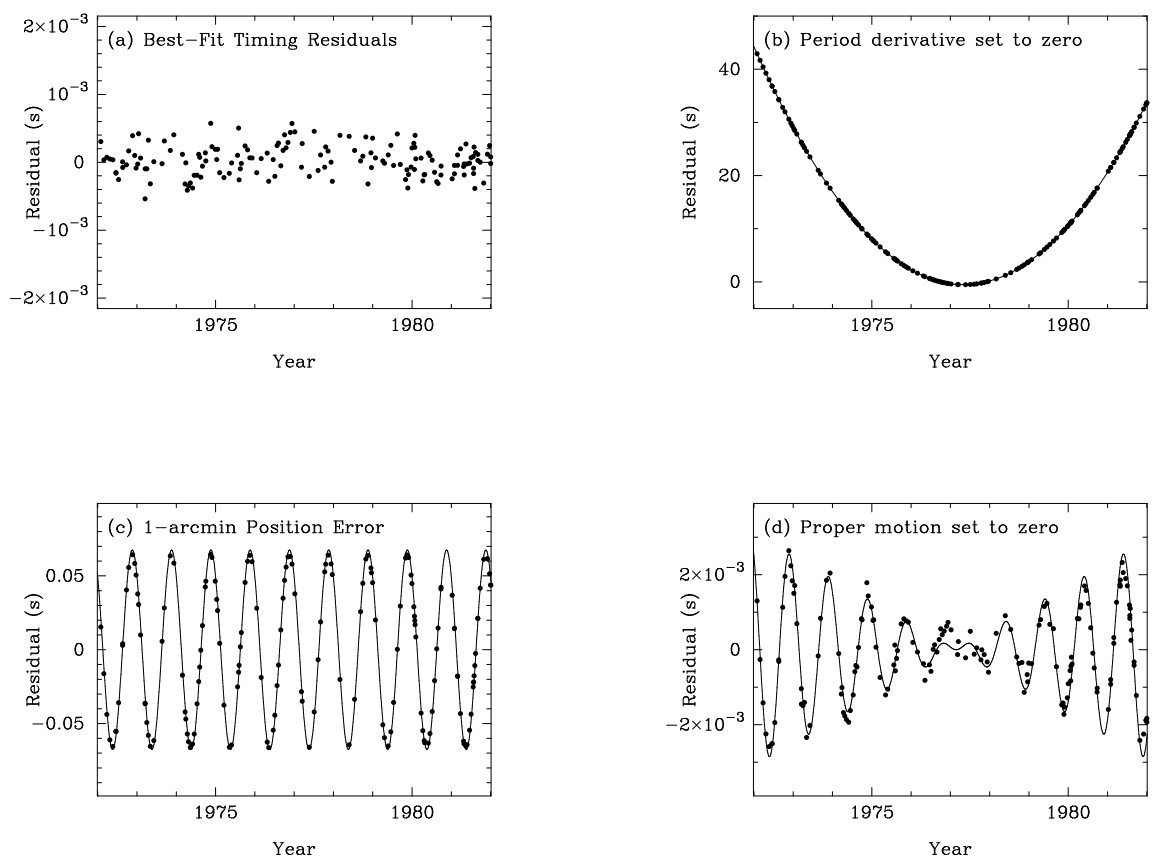

Figure 19: Timing model residuals versus date for PSR B1133+16. Case (a) shows the residuals obtained from the best fitting model which includes period, the period derivative, position and proper motion. Case (b) is the result of setting the period derivative term to zero in this model. Case (c) shows the effect of a 1 arcmin error in the assumed declination. Case (d) shows the residuals obtained assuming zero proper motion. The lines in $(b)-(d)$ show the expected behaviour in the timing residuals for each effect (see text). 
used in the barycentric time calculation is incorrect. A position error of just one arcsecond results in an annual sinusoid (Fig. 19c) with a peak-to-peak amplitude of about $5 \mathrm{~ms}$ for a pulsar on the ecliptic; this is easily measurable for typical TOA uncertainties of order one milliperiod or better. A proper motion produces an annual sinusoid of linearly increasing magnitude (Fig. 19d).

After a number of iterations, and with the benefit of a modicum of experience, it is possible to identify and account for each of these various effects to produce a "timing solution" which is phase coherent over the whole data span. The resulting model parameters provide spin and astrometric information about the neutron star to a precision which improves as the length of the data span increases. Timing observations of the original millisecond pulsar B1937+21, spanning almost 9 years (exactly 165,711,423,279 rotations!), measure a period of $1.5578064688197945 \pm 0.000000000000004 \mathrm{~ms}[117,114]$ defined at midnight UT on December 5 1988! Astrometric measurements based on these data are no less impressive, with position errors of $\sim 20 \mu$ arcsec being presently possible.

\subsection{Timing stability}

Ideally, after correctly applying a timing model, we would expect a set of uncorrelated timing residuals scattered in a Gaussian fashion about a zero mean with an rms consistent with the measurement uncertainties. This is not always the case; the residuals of many pulsars exhibit a quasi-periodic wandering with time.

A number of examples are shown in Fig. 20. These are taken from the Jodrell Bank timing program [216]. Such "timing noise" is most prominent in the youngest of the normal pulsars $[162,59]$ and virtually absent in the much older millisecond pulsars [117]. While the physical processes of this phenomena are not well understood, it seems likely that it may be connected to superfluid processes and temperature changes in the interior of the neutron star [3], or processes in the magnetosphere [55, 54].

The relative dearth of timing noise for the older pulsars is a very important finding. It implies that, presently, the measurement precision depends primarily on the particular hardware constraints of the observing system. Consequently, a large effort in hardware development is presently being made to improve the precision of these observations using, in particular, coherent dedispersion outlined in $\S 4.1$. Much of the pioneering work in this area has been made by Joseph Taylor and collaborators at Princeton University [196]. From high quality observations made using the Arecibo radio telescope spanning almost a decade [206, 207, 117], the group has demonstrated that the timing stability of millisecond pulsars over such time-scales is comparable to terrestrial atomic clocks.

This phenomenal stability is demonstrated in Fig. 21. This figure shows $\sigma_{z}$, a parameter closely resembling the Allan variance used by the clock community to estimate the stability of atomic clocks $[233,1]$. Atomic clocks are known to have $\sigma_{z} \sim 5 \times 10^{-15}$ on time-scales of order 5 years. The timing stability of PSR B1937+21 seems to be limited by a power law component which produces

Living Reviews in Relativity (2001-5)

http://www.livingreviews.org 


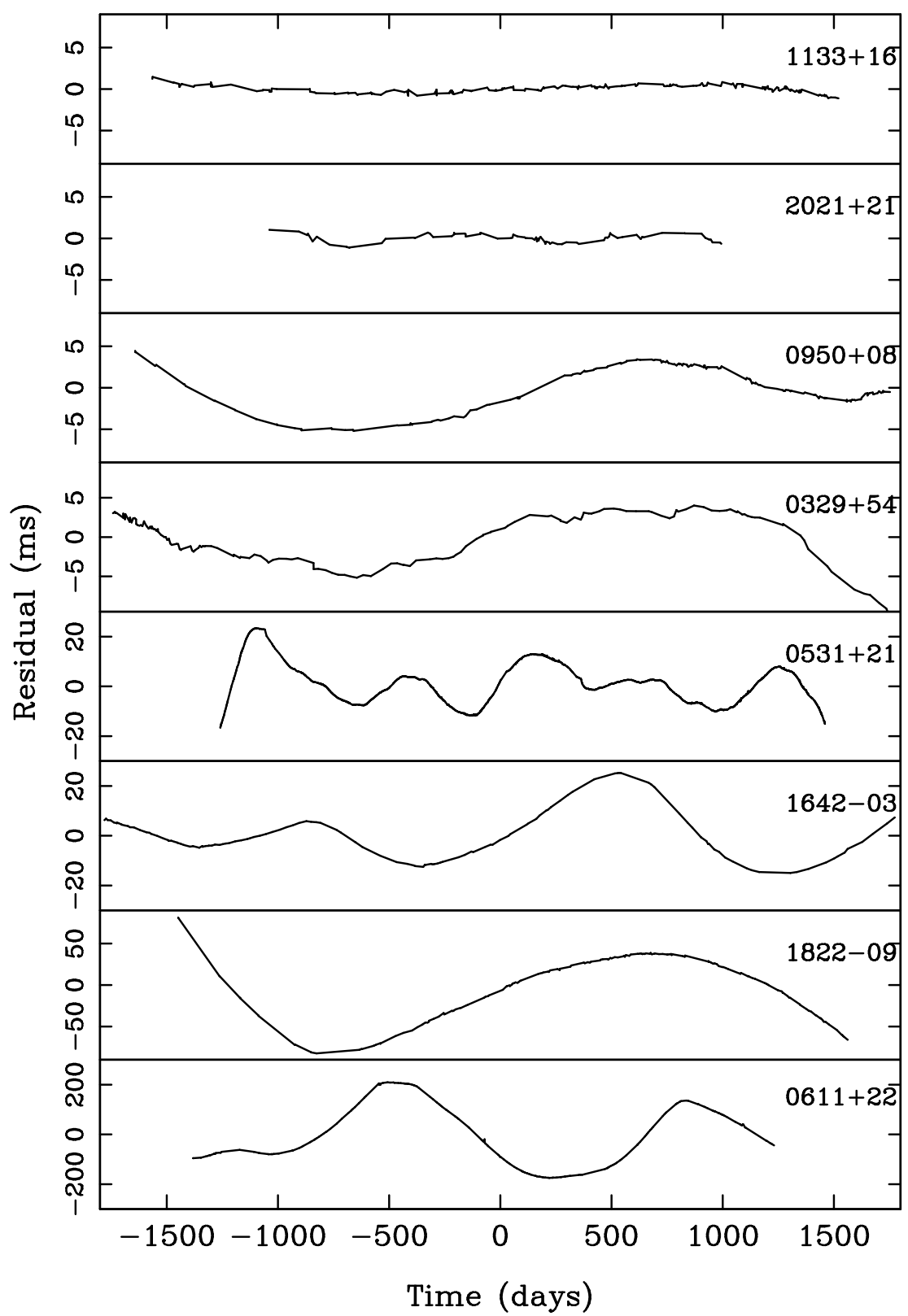

Figure 20: Examples of timing residuals for a number of normal pulsars. Note the varying scale on the ordinate axis, the pulsars being ranked in increasing order of timing "activity". 


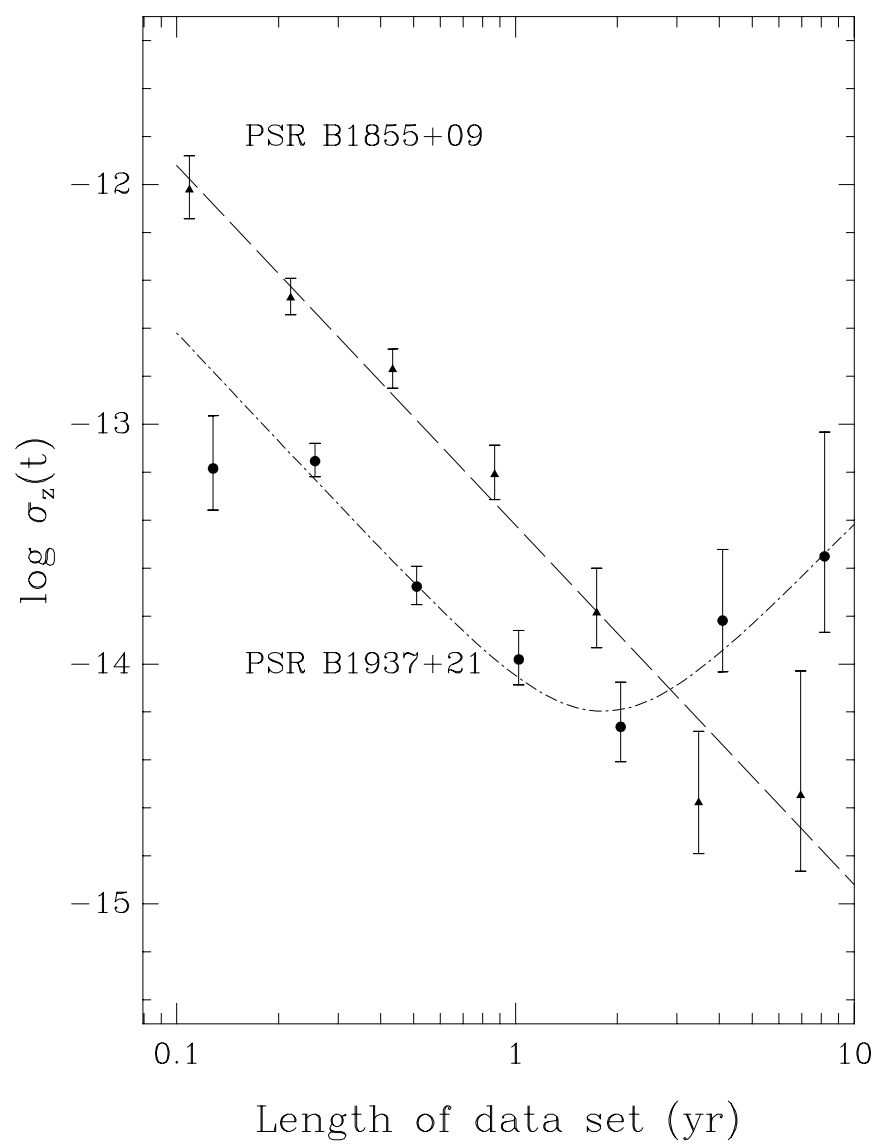

Figure 21: Fractional timing instabilities for PSRs B1855+09 and B1937+21 as a function of time. (After Kaspi, Taylor $\mathcal{G}$ Ryba 1994 [117].) 
a minimum in its $\sigma_{z}$ after $\sim 2 \mathrm{yr}$. This is most likely a result of a small amount of intrinsic timing noise [117]. No such noise component is observed for PSR B1855+09. This demonstrates that the timing stability for PSR B1855+09 becomes competitive with the atomic clocks after about $3 \mathrm{yr}$. The absence of timing noise for B1855+09 is probably related to its characteristic age $\sim 5 \mathrm{Gyr}$ which is about a factor of 20 larger than B1937+21. Timing observations of millisecond pulsars are discussed further in the context of the pulsar timing array in $\S 5.2$.

\subsection{Binary pulsars and Kepler's laws}

For binary pulsars, the simple timing model introduced in $\S 4.2$ needs to be extended to incorporate the additional radial acceleration of the pulsar as it orbits the common centre-of-mass of the binary system. Treating the binary orbit using Kepler's laws to refer the TOAs to the binary barycentre requires five additional model parameters: the orbital period $\left(P_{\mathrm{b}}\right)$, projected semi-major orbital axis $\left(a_{\mathrm{p}} \sin i\right.$, see below), orbital eccentricity $(e)$, longitude of periastron $(\omega)$ and the epoch of periastron passage $\left(T_{0}\right)$. This description, using five "Keplerian parameters", is identical to that used for spectroscopic binary stars.

For spectroscopic binaries the orbital velocity curve shows the radial component of the star's velocity as a function of time. The analogous plot for pulsars is the apparent pulse period against time. Two examples are given in Fig. 22.

Constraints on the mass of the orbiting companion can be placed by combining the projected semi-major axis $a_{\mathrm{p}} \sin i$ and the orbital period to obtain the mass function:

$$
f\left(m_{\mathrm{p}}, m_{\mathrm{c}}\right)=\frac{4 \pi^{2}}{G} \frac{\left(a_{\mathrm{p}} \sin i\right)^{3}}{P_{\mathrm{b}}^{2}}=\frac{\left(m_{\mathrm{c}} \sin i\right)^{3}}{\left(m_{\mathrm{p}}+m_{\mathrm{c}}\right)^{2}},
$$

where $G$ is the universal gravitational constant. Assuming a pulsar mass $m_{\mathrm{p}}$ of $1.35 M_{\odot}$ (see below), the mass of the orbiting companion $m_{\mathrm{c}}$ can be estimated as a function of the (initially unknown) angle $i$ between the orbital plane and the plane of the sky. The minimum companion mass $m_{\min }$ occurs when the orbit is assumed edge-on $\left(i=90^{\circ}\right)$. For a random distribution of orbital inclination angles, the probability of observing a binary system at an angle less than some value $i_{0}$ is $p\left(<i_{0}\right)=1-\cos \left(i_{0}\right)$. This implies that the chances of observing a

binary system inclined at an angle $\lesssim 26^{\circ}$ is only $10 \%$; evaluating the companion mass for this inclination angle $m_{90}$ constrains the mass range between $m_{\min }$ and $m_{90}$ at the $90 \%$ confidence level.

\subsection{Post-Keplerian parameters}

Although many of the presently known binary pulsar systems can be adequately described by a Keplerian orbit, there are several systems, including the original binary pulsar B1913+16, which exhibit relativistic effects that require an additional set of up to five "post-Keplerian" parameters. Within the framework of 
PSR $1012+5307$
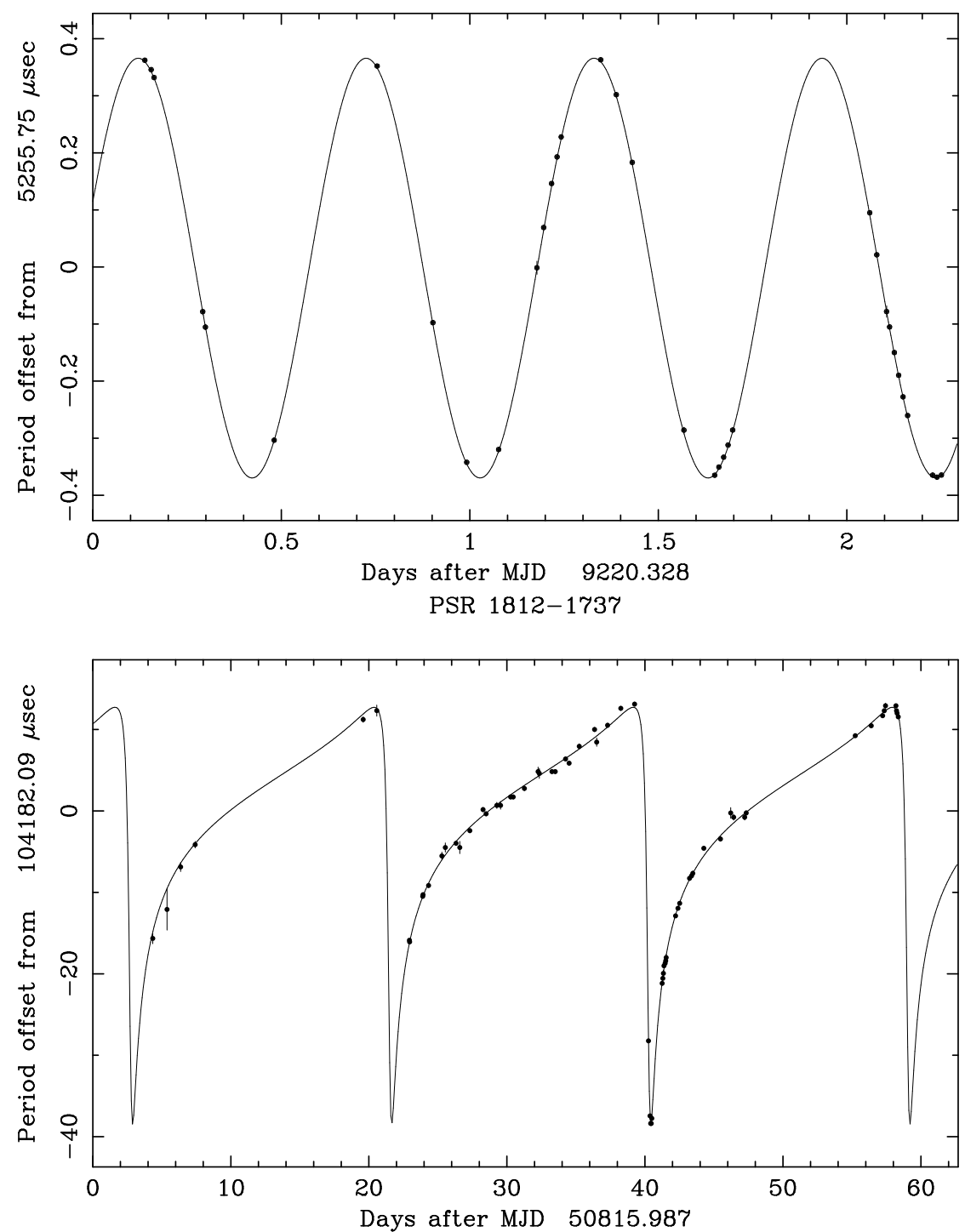

Figure 22: Orbital velocity curves for two binary pulsars. Left: PSR J1012+5307, a 5.25-ms pulsar in a 14.5-hour circular orbit around a low-mass white dwarf companion [17\%, 256, 125]. Right: PSR J1811-1736, a 104-ms pulsar in a highly eccentric 18.8-day orbit around a massive companion (probably another neutron star) [147].

Living Reviews in Relativity (2001-5)

http://www.livingreviews.org 
general relativity, these can be written [37] as:

$$
\begin{gathered}
\dot{\omega}=3\left(\frac{P_{\mathrm{b}}}{2 \pi}\right)^{-5 / 3}\left(T_{\odot} M\right)^{2 / 3}\left(1-e^{2}\right)^{-1} \\
\gamma=e\left(\frac{P_{\mathrm{b}}}{2 \pi}\right)^{1 / 3} T_{\odot}^{2 / 3} M^{-4 / 3} m_{\mathrm{c}}\left(m_{\mathrm{p}}+2 m_{\mathrm{c}}\right) \\
\dot{P}_{\mathrm{b}}=-\frac{192 \pi}{5}\left(\frac{P_{\mathrm{b}}}{2 \pi}\right)^{-5 / 3}\left(1+\frac{73}{24} e^{2}+\frac{37}{96} e^{4}\right)\left(1-e^{2}\right)^{-7 / 2} T_{\odot}^{5 / 3} m_{\mathrm{p}} m_{\mathrm{c}} M^{-1 / 3} \\
r=T_{\odot} m_{\mathrm{c}} \\
s=x\left(\frac{P_{\mathrm{b}}}{2 \pi}\right)^{-2 / 3} T_{\odot}^{-1 / 3} M^{2 / 3} m_{\mathrm{c}}^{-1}
\end{gathered}
$$

In addition to the symbols defined above for Equation (10), $M \equiv m_{\mathrm{p}}+m_{\mathrm{c}}$, $x \equiv a_{\mathrm{p}} \sin i / c, s \equiv \sin i$ and $T_{\odot} \equiv G M_{\odot} / c^{3} \simeq 4.925 \mu \mathrm{s}$. All masses are in solar units.

Measurements of post-Keplerian parameters for PSR B1913+16 have been carried out by Taylor and a number of collaborators over the years with steadily improving precision. The first of these parameters to be measured was the advance of the longitude of periastron $\dot{\omega}$. This measurement is analogous to the perihelion advance of Mercury [169]. For PSR B1913+16 this amounts to about 4.2 degrees per year [237], some 4.6 orders of magnitude larger than for Mercury. A measurement of $\dot{\omega}$ alone yields the total mass for this system, $M=2.83 M_{\odot}$, assuming this advance is due to general relativity.

Measurement of a second post-Keplerian parameter for B1913+16, $\gamma$ (gravitational redshift and transverse Doppler shifts in the orbit), permits an unambiguous determination of $m_{\mathrm{p}}, m_{\mathrm{c}}$ and $i$ when combined with $\dot{\omega}$ and the five Keplerian parameters. The original measurements [236] have since been substantially refined $[239,240]$ and the mass of the pulsar and its unseen companion have been determined to be $1.442 \pm 0.003 M_{\odot}$ and $1.386 \pm 0.003 M_{\odot}$ respectively. Such phenomenal precision is a testament to the timing stability of radio pulsars as clocks, and the diligence of Taylor and collaborators in carrying out these long-term measurements. Similar mass measurements now exist for the two other double neutron star binary systems discussed in $\S 3.4 .1$ : B1534+12 [220] and B2127+11C [66]. For a number of other systems, $\dot{\omega}$ measurements allow interesting constraints to be placed on the component masses [246, 179, 248].

An important general relativistic prediction for eccentric double neutron star systems is the orbital decay due to the emission of gravitational radiation $\left(\dot{P}_{\mathrm{b}}\right.$ in Equation (13)). Taylor et al. [236, 239, 240] were able to measure this for B1913+16 and found it to be in excellent agreement with the predicted value.

Living Reviews in Relativity (2001-5) http://www. livingreviews.org 


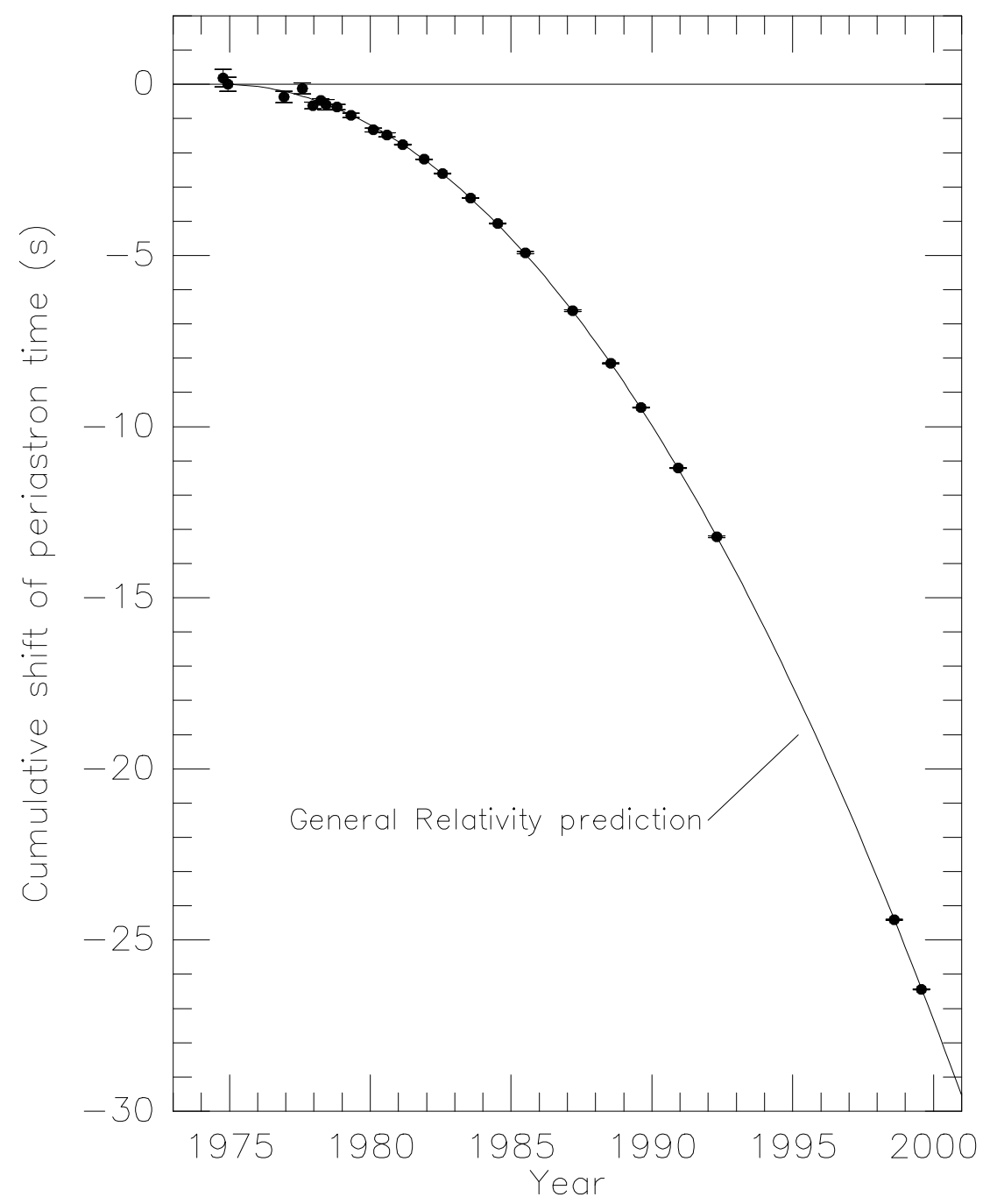

Figure 23: Orbital decay in the binary pulsar B1913+16 system demonstrated as an increasing orbital phase shift for periastron passages with time. The general relativistic prediction due entirely to the emission of gravitational radiation is shown by the parabola.

Living Reviews in Relativity (2001-5)

http://www.livingreviews.org 
The orbital decay, which corresponds to a shrinkage of about $3.2 \mathrm{~mm}$ per orbit, is seen most dramatically as the gradually increasing shift in orbital phase for periastron passages with respect to a non-decaying orbit shown in Fig. 23. This figure includes recent Arecibo data taken in 1998 and 1999 following the upgrade of the telescope in the mid 1990s. The observations of the orbital decay, now spanning a 25-year baseline, are in agreement with general relativity at the level of about $0.5 \%$ and provide the first (indirect) evidence for the existence of gravitational radiation. Hulse and Taylor were awarded the Nobel prize in Physics in 1993 [241, 101, 234] in recognition of their discovery of this remarkable laboratory for testing general relativity.

For those binary systems which are oriented nearly edge-on to the line-ofsight, a significant delay is expected for orbital phases around superior conjunction where the pulsar radiation is bent in the gravitational potential well of the companion star. The so-called "range" and "shape" of the Shapiro delay effect are parameterized by the last two post-Keplerian parameters $r$ and $s \equiv \sin i$ that were introduced in Equations (14) and (15). This effect, analogous to the solar system Shapiro delay, has so far been measured for two neutron star-white dwarf binary systems, B1855+09 and J1713+0747 [206, 117, 46], and for the double neutron star binaries B1534+12 [220] and B1913+16 [240].

\subsection{Geodetic precession}

Shortly after the discovery of PSR B1913+16 it was realized that, if the spin axis of the visible pulsar was misaligned with the angular momentum axis of the binary system, the perturbing effect of the companion on the space-time around the radio pulsar would cause it to precess around the angular momentum axis $[63,76]$. Within the framework of general relativity, the rate of precession $\Omega_{\mathrm{p}}$ was shown [22] to be

$$
\Omega_{\mathrm{p}}=\frac{(2 \pi)^{5 / 3} T_{\odot}^{2 / 3} m_{\mathrm{c}}\left(4 m_{\mathrm{p}}+3 m_{\mathrm{c}}\right)}{P_{\mathrm{b}}^{5 / 3}\left(m_{\mathrm{p}}+m_{\mathrm{c}}\right)^{4 / 3}\left(1-e^{2}\right)},
$$

where we assume the same notation used for the discussion in $\S 4.4$ and $\S 4.5$. Inserting the parameters of PSR B1913+16 yields $\Omega_{\mathrm{p}}=1.21 \mathrm{deg} \mathrm{yr}^{-1}$. The period of the precession is $297.5 \mathrm{yr}$. The observational consequence of geodetic precession is a secular change in the pulse profile as the line-of-sight cut through the emission beam changes (recall Fig. 5).

Early qualitative evidence for profile evolution due to this effect [236] was substantiated with long-term Arecibo measurements of component changes by Weisberg et al. [264]. Further changes were seen by Kramer with new Effelsberg data acquired in the 1990s [119]. In addition to relative amplitude variations, the expected changes in component separation for a hollow-cone beam model were also seen in the Effelsberg data. These observations are summarized in Fig. 24.

In addition to the above results, there is now evidence for geodetic precession in the other classic neutron star binary, PSR B1534+12 [9, 223]. Although 
geodetic precession in binary pulsars is another successful test of general relativity (albeit at a lower precision than e.g. orbital decay measurements), what is perhaps more interesting are the various consequences it has. Geodetic precession only occurs when the spin and orbital axes are misaligned [22]. This is most likely to occur if the neutron star received an impulsive "kick" velocity at birth (§ 2.4.4). Wex et al. [265] have investigated the B1913+16 observations and find that the kick magnitude was at least $250 \mathrm{~km} \mathrm{~s}^{-1}$ and was directed almost perpendicular to the spin axis of the neutron star progenitor. This places stringent constraints on any kick mechanism. Detailed monitoring of the pulse profile and polarization properties now underway [262, 120] will allow the first map of the emission beam of a neutron star to be made. This has important implications for the various beaming models described in $\S 3.2 .3$. There are already indications that the beam is circular [120].

The current results predict that B1913+16 will completely precess out of the line of sight by around 2025 and re-appear some 240 years later [119]. Although we shall lose a most treasured pulsar, we can take comfort from the fact that other pulsars will precess into our field of view. Perhaps one example is the newly-discovered relativistic binary J1141-6545 [116] discussed in $\S 2.6 .2$ and $\S 3.4 .2$. This relatively bright object was apparently missed by two previous searches during the early 1990s [109, 160, 152].

\subsection{Going further}

This chapter has outlined past and present progress in a number of areas related to pulsar timing. For further details on the technical details and prospects of pulsar timing, the interested reader is referred to a number of excellent review articles [269, 16, 233, 23, 24]. Two freely available software packages which are routinely used for time-of-arrival analyses by the pulsar community are available, viz. TEMPO $[240,196]$ and TIMAPR $[72,166]$. These packages are based on more detailed versions of the timing model outlined in $\S 4.2$. An up-to-date list summarising the various timing programmes is kept by Don Backer [10]. An audio file and slides from a lecture on pulsar timing presented by Backer at the centennial meeting of the American Physical society is also available online [11]. Another relevant lecture from that meeting is Will's presentation [268] on tests of Einstein's relativity which includes an excellent overview of Taylor and Weisberg's measurements of PSR B1913+16. Kramer [120] has written a lucid review article discussing measurements of geodetic precession in binary pulsars and their implications.

The remarkable precision of these measurements, particularly for millisecond pulsars, allows the detection of radial accelerations on the pulsar induced by orbiting bodies smaller than the Earth. Alex Wolszczan detected one such "pulsar planetary system" in 1990 following the discovery of a 6.2-ms pulsar B1257+12. In this case, the pulsar is orbited by at least three Earth-mass bodies [273, 186, 272]. Subsequent measurements of B1257+12 were even able to measure resonance interactions between two of the planets [271], confirming the nature of the system beyond all doubt. Long-term timing measurements

Living Reviews in Relativity (2001-5)

http://www.livingreviews.org 

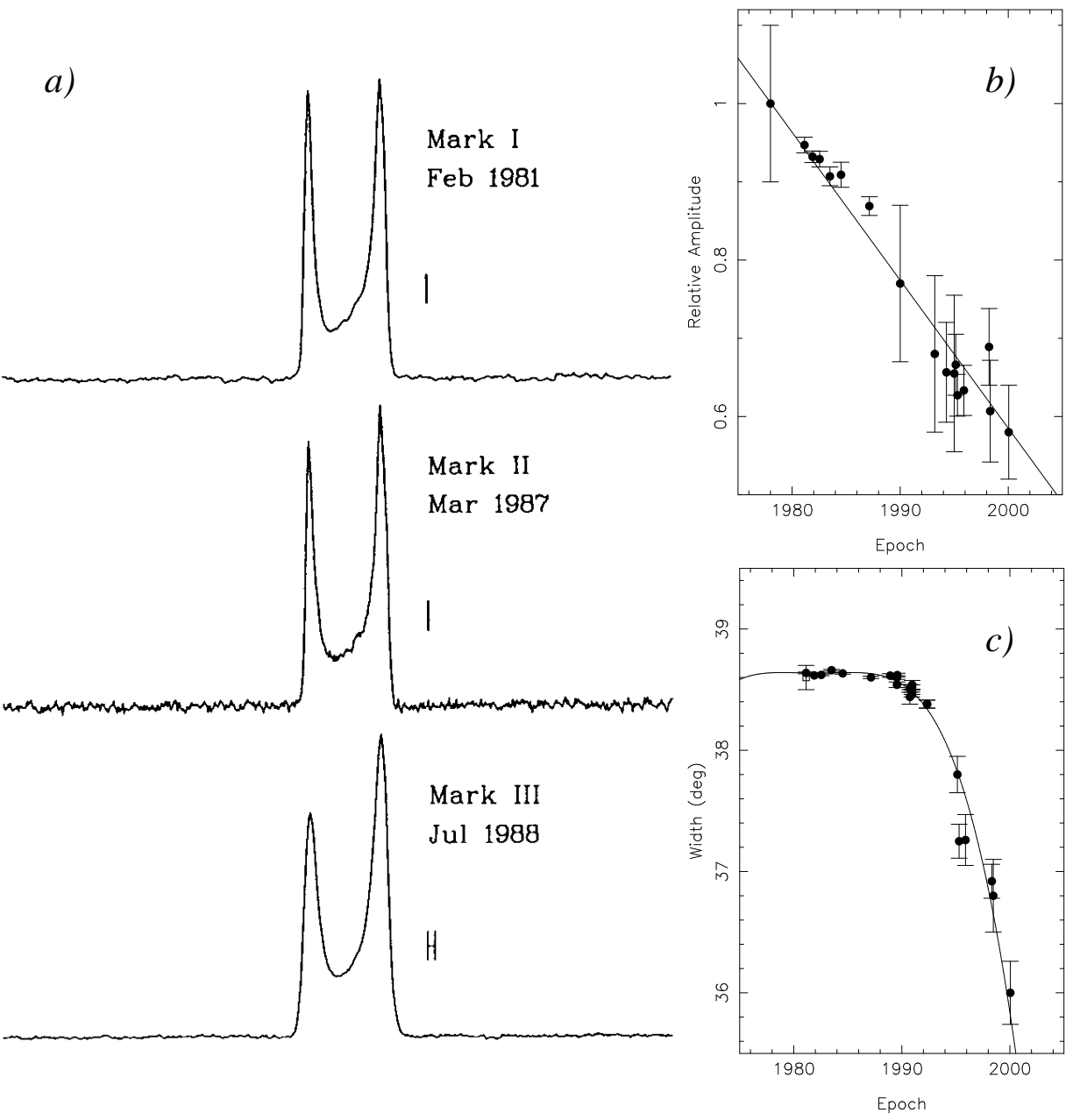

Figure 24: Geodetic precession in the binary pulsar B1913+16 system: (a) Changes in the observed pulse shapes between 1981-1988 seen as a decrease in amplitude between the left and right components in the pulse profile [240, 264]; (b) relative heights of the two amplitudes plotted between 1975-1998 [119]; (c) component separation change [119]. 
of the 11-ms pulsar B1620-26 in the globular cluster M4 indicate that it may also have a planetary companion [247, 15, 245]. For detailed reviews of these systems, and their implications for planetary formation scenarios, the interested reader is referred to [189].

Living Reviews in Relativity (2001-5)

http://www.livingreviews.org 


\section{Pulsars as Gravitational Wave Detectors}

Many cosmological models predict that the Universe is presently filled with a stochastic gravitational wave background (GWB) produced during the big bang era [185]. The idea to use pulsars as natural detectors of gravitational waves was first explored independently by both Sazhin and Detweiler in the late 1970s [209, 68]. The basic concept is to treat the solar system barycentre and a distant pulsar as opposite ends of an imaginary arm in space. The pulsar acts as the reference clock at one end of the arm sending out regular signals which are monitored by an observer on the Earth over some time-scale $T$. The effect of a passing gravitational wave would be to cause a change in the observed rotational frequency by an amount proportional to the amplitude of the wave. For regular monitoring observations of a pulsar with typical TOA uncertainties of $\epsilon_{\mathrm{TOA}}$, this "detector" would be sensitive to waves with dimensionless amplitudes $\gtrsim \epsilon_{\mathrm{TOA}} / T$ and frequencies as low as $\sim 1 / T[29,36]$. This method, which already yields interesting upper limits on the GWB, is reviewed in $\S 5.1$. The idea of a more sensitive detector based on an array of pulsar clocks distributed over the sky is discussed in $\S 5.2$.

\subsection{Limits from individual pulsars}

In the ideal case, the change in the observed frequency caused by the GWB should be detectable in the set of timing residuals after the application of an appropriate model for the rotational, astrometric and, where necessary, binary parameters of the pulsar. As discussed in $\S 4$, all other effects being negligible, the rms scatter of these residuals $\sigma$ would be due to the measurement uncertainties and intrinsic timing noise from the neutron star. Detweiler [68] showed that a GWB with a flat energy spectrum in the frequency band $f \pm f / 2$ would result in an additional contribution to the timing residuals $\sigma_{\mathrm{g}}$. The corresponding wave energy density $\rho_{\mathrm{g}}($ for $f T \gg 1$ ) is

$$
\rho_{\mathrm{g}}=\frac{243 \pi^{3} f^{4} \sigma_{\mathrm{g}}^{2}}{208 G}
$$

An upper limit to $\rho_{\mathrm{g}}$ can be obtained from a set of timing residuals by assuming the rms scatter is entirely due to this effect $\left(\sigma=\sigma_{\mathrm{g}}\right)$. These limits are commonly expressed as a fraction of $\rho_{\mathrm{c}}$ the energy density required to close the Universe:

$$
\rho_{\mathrm{c}}=\frac{3 H_{0}^{2}}{8 \pi G} \simeq 2 \times 10^{-29} h^{2} \mathrm{~g} \mathrm{~cm}^{-3},
$$

where the Hubble constant $H_{0}=100 h \mathrm{~km} \mathrm{~s}^{-1}$ Mpc.

Romani \& Taylor [205] applied this technique to a set of TOAs for PSR B1237+12 obtained from regular observations over a period of 11 years as part of the JPL pulsar timing programme [73]. This pulsar was chosen on the basis of its relatively low level of timing activity by comparison with the youngest pulsars, whose residuals are ultimately plagued by timing noise (§ 4.3). By ascribing

Living Reviews in Relativity (2001-5) http://www.livingreviews.org 
the rms scatter in the residuals $(\sigma=240 \mathrm{~ms})$ to the GWB, Romani \& Taylor placed a limit of $\rho_{\mathrm{g}} / \rho_{\mathrm{c}} \lesssim 4 \times 10^{-3} h^{-2}$ for a centre frequency $f=7 \times 10^{-9} \mathrm{~Hz}$.

This limit, already well below the energy density required to close the Universe, was further reduced following the long-term timing measurements of millisecond pulsars at Arecibo by Taylor and collaborators (§ 4.3). In the intervening period, more elaborate techniques had been devised [29, 36, 228] to look for the likely signature of a GWB in the frequency spectrum of the timing residuals and to address the possibility of "fitting out" the signal in the TOAs. Following [29] it is convenient to define $\Omega_{\mathrm{g}}$, the energy density of the GWB per logarithmic frequency interval relative to $\rho_{\mathrm{c}}$. With this definition, the power spectrum of the GWB, $\mathcal{P}(f)$, can be written $[99,36]$ as

$$
\mathcal{P}(f)=\frac{G \rho_{\mathrm{g}}}{3 \pi^{3} f^{4}}=\frac{H_{0}^{2} \Omega_{\mathrm{g}}}{8 \pi^{4} f^{5}}=1.34 \times 10^{4} \Omega_{\mathrm{g}} h^{2} f_{\mathrm{yr}^{-1}}^{-5} \mu \mathrm{s}^{2} \mathrm{yr},
$$

where $f_{\mathrm{yr}}^{-1}$ is frequency in cycles per year. The timing residuals for B1937+21 shown in Fig. 25 are clearly non-white and, as we saw in $\S 4.3$, limit its timing stability for periods $\gtrsim 2 \mathrm{yr}$. The residuals for PSR B1855+09 clearly show no systematic trends and are in fact consistent with the measurement uncertainties alone. Based on these data, and using a rigorous statistical analysis, Thorsett \& Dewey [249] place a $95 \%$ confidence upper limit of $\Omega_{\mathrm{g}} h^{2}<10^{-8}$ for $f=$ $4.4 \times 10^{-9} \mathrm{~Hz}$. This limit is difficult to reconcile with most cosmic string models for galaxy formation [40, 249].

For those pulsars in binary systems, an additional clock for measuring the effects of gravitational waves is the orbital period. In this case, the range of frequencies is not limited by the time span of the observations, allowing the detection of waves with periods as large as the light travel time to the binary system [29]. The most stringent results presently available are based on the B1855+09 limit $\Omega_{\mathrm{g}} h^{2}<2.7 \times 10^{-4}$ in the frequency range $10^{-11}<f<4.4 \times$ $10^{-9} \mathrm{~Hz}$. Kopeikin [118] has recently presented this limit and discusses the methods in detail.

\subsection{A pulsar timing array}

The idea of using timing data for a number of pulsars distributed on the sky to detect gravitational waves was first proposed by Hellings \& Downs [96]. Such a "timing array" of pulsars would have the advantage over a single arm in that, through a cross-correlation analysis of the residuals for pairs of pulsars distributed over the sky, it should be possible to separate the timing noise of each pulsar from the signature of the GWB, which would be common to all pulsars in the array. To quantify this, consider the fractional frequency shift observed for the $i$ th pulsar in the array:

$$
\frac{\delta \nu_{i}}{\nu_{i}}=\alpha_{i} \mathcal{A}(t)+\mathcal{N}_{i}(t)
$$

In this expression $\alpha_{i}$ is a geometric factor dependent on the line-of-sight direction to the pulsar and the propagation and polarisation vectors of the gravitational

Living Reviews in Relativity (2001-5)

http://www.livingreviews.org 

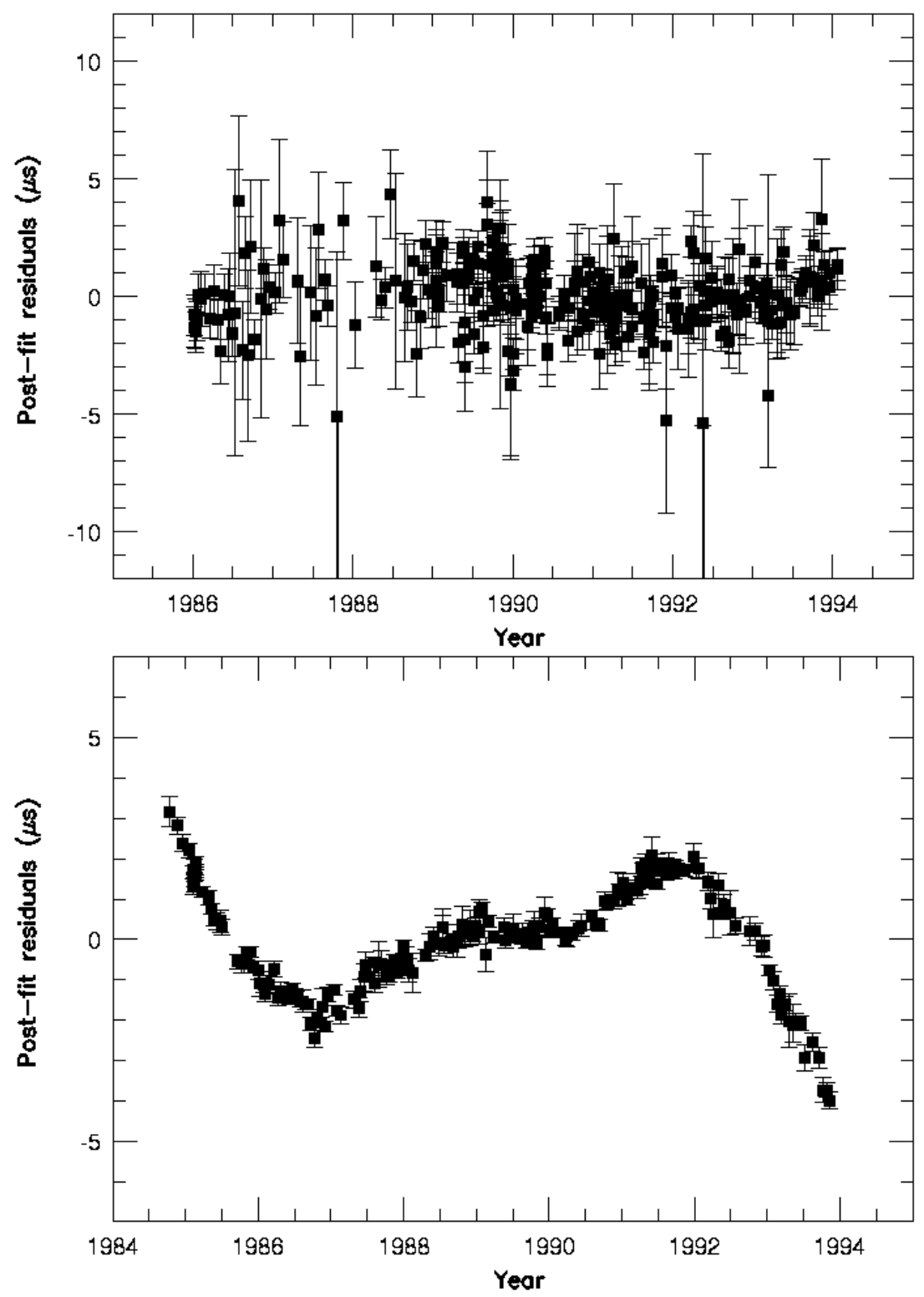

Figure 25: Timing residuals for PSRs B1855+09 (left panel) and B1937+21 (right panel) obtained from almost a decade of timing at Arecibo (Kaspi, Taylor \& Ryba 1994 [117]). 
wave of dimensionless amplitude $\mathcal{A}$. The timing noise intrinsic to the pulsar is characterised by the function $\mathcal{N}_{i}$. The result of a cross-correlation between pulsars $i$ and $j$ is then

$$
\alpha_{i} \alpha_{j}\left\langle\mathcal{A}^{2}\right\rangle+\alpha_{i}\left\langle\mathcal{A} \mathcal{N}_{j}\right\rangle+\alpha_{j}\left\langle\mathcal{A} \mathcal{N}_{i}\right\rangle+\left\langle\mathcal{N}_{i} \mathcal{N}_{j}\right\rangle,
$$

where the bracketed terms indicate cross-correlations. Since the wave function and the noise contributions from the two pulsars are independent quantities, the cross correlation tends to $\alpha_{i} \alpha_{j}\left\langle\mathcal{A}^{2}\right\rangle$ as the number of residuals becomes large. Summing the cross-correlation functions over a large number of pulsar pairs provides additional information on this term as a function of the angle on the sky [95]. This allows the separation of the effects of terrestrial clock and solar system ephemeris errors from the GWB [79].

Applying the timing array concept to the present database of long-term timing observations of millisecond pulsars does not improve on the limits on the GWB discussed above. The sky distribution of these pulsars, seen in the left panel of Fig. 26, shows that their angular separation is rather low. To achieve optimum sensitivity it is desirable to have an array consisting of pulsar clocks distributed isotropically over the whole sky. The flood of recent discoveries of nearby binary and millisecond pulsars has resulted in essentially such a distribution, shown in the right panel of Fig. 26.
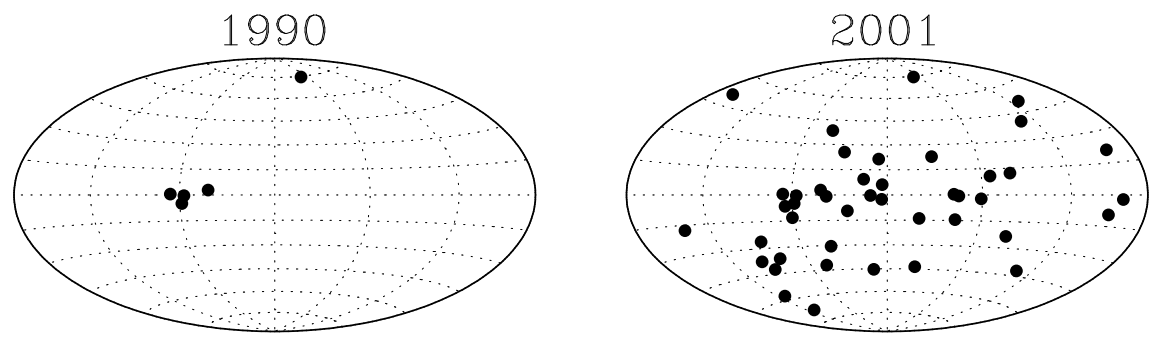

Figure 26: Hammer-Aitoff projections showing the known Galactic disk millisecond pulsar population in 1990 and 2001. The impact of the new discoveries is seen by comparing the sample circa 1990, where all the known sources had been discovered at Arecibo, with the present sample where the sources are much more uniformly distributed on the sky.

A number of long-term timing projects are now underway to monitor these millisecond pulsars with a goal of detecting low-frequency gravitational radiation. At Arecibo, regular timing of a dozen or more millisecond pulsars has been carried out following the completion of the upgrade to the telescope in 1997. A summary of these observations is shown in Fig. 27. The rms timing residuals for several of the pulsars are now approaching the $100 \mathrm{~ns}$ level. This degree of precision demands a high level of commitment to investigate possible causes of systematic errors in the signal path through the telescope. Combining datasets from several observatories is also challenging. The Berkeley pulsar group lead by Don Backer [10] are among the most active observers in this

Living Reviews in Relativity (2001-5)

http://www.livingreviews.org 
area. Backer and collaborators have now installed identical sets of datataking equipment at a number of radio telescopes around the world in an attempt to ensure a homogeneous set of residuals. Continued timing of these millisecond pulsars in the coming years should greatly improve the sensitivity and will perhaps allow the detection of gravitational waves, as opposed to upper limits, in the not-too-distant future.

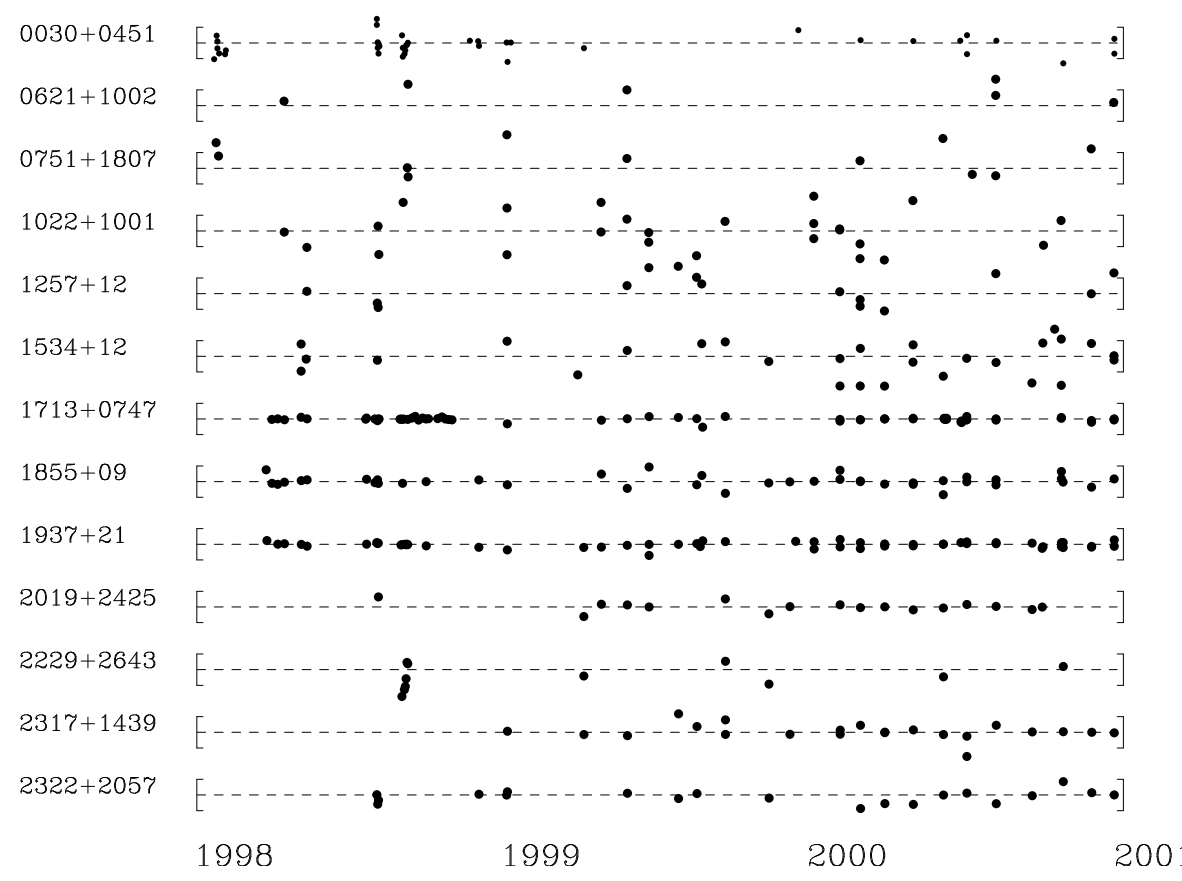

Figure 27: Arecibo timing residuals of millisecond pulsars monitored by groups at Berkeley and Princeton on a regular basis as part of a long-term project to detect low-frequency gravitational waves. The level of precision being achieved is well below the vertical bars surrounding each set of residuals $( \pm 5 \mu \mathrm{s})$.

\subsection{Going further}

Further discussions on the realities of using pulsars as gravity wave detectors can be found in two excellent review articles by Romani [203] and Backer [12]. 


\section{$6 \quad$ Summary and Future Prospects}

The main aim of this article was to review some of the many astrophysical applications provided by the present sample of binary and millisecond radio pulsars. The topics covered here, along with the bibliography and associated tables of observational parameters, should be useful to those wishing to delve deeper into the vast body of literature that exists. We now briefly recap on the main issues.

Through an understanding of the Galactic population of radio pulsars summarised in $\S 3$ it is possible to predict the detection statistics of terrestrial gravitational wave detectors to nearby rapidly spinning neutron stars (§3.3), as well as coalescing relativistic binaries at cosmic distances ( $\S 3.4)$. Continued improvements in gravitational wave detector sensitivities should result in a number of interesting developments and contributions in this area. These developments and contributions might include the detection of presently known radio pulsars, as well as a population of coalescing binary systems which have not yet been detected as radio pulsars. The phenomenal timing stability of radio pulsars leads naturally to a large number of applications, including their use as laboratories for relativistic gravity $(\S 4.5)$ and as natural detectors of gravitational radiation (§5). Long-term timing experiments of the present sample of millisecond and binary pulsars currently underway appear to have tremendous potential in these areas and perhaps detect the gravitational wave background (if it exists) within the next decade.

These applications will benefit greatly from the continued discovery of new systems by the present generation of radio pulsar searches which continue to probe new areas of parameter space. Based on the results presented in $\S 3.3$, it is clear that we are aware of only about $1 \%$ of the total active pulsar population in our Galaxy. It is therefore likely that we have not seen all of the pulsar zoo. More sensitive surveys are being planned both in the short term (a multibeam system on the Arecibo telescope [174]) and in the longer term (the Square Kilometer Array [242]). These should provide a far more complete census of the Galactic pulsar population. Possible discoveries in the future include:

- A dual-line binary pulsar, i.e. a double neutron star system in which both components are observable as radio pulsars. The additional clock in such a binary system would be most valuable in further tests of strong-field gravity.

- A radio pulsar with a black-hole companion would undoubtably also be a fantastic laboratory for studying gravity in the strong-field regime.

- A sub-millisecond pulsar. The original millisecond pulsar, B1937+21, rotating at $642 \mathrm{~Hz}$ is still the most rapidly rotating neutron star known. Do $\mathrm{kHz}$ neutron stars exist? Searches now have sensitivity to such objects [39] and a discovery of even one would constrain the equation of state of matter at high densities.

Living Reviews in Relativity (2001-5)

http://www.livingreviews.org 
- A binary system in which the neutron star is in the process of transforming from an X-ray-emitting neutron star to a millisecond radio pulsar.

Spurred on by recent discoveries [47, 154, 199, 62], a number of high-sensitivity searches for pulsars in globular clusters are being conducted. These have tremendous potential for discovering new and exotic binary systems like a millisecond pulsar-black hole binary.

\subsection{Acknowledgments}

Many thanks to Maura McLaughlin, Jiannis Seiradakis and Michael Kramer who read and commented on earlier incantations of this revised review, as well as a number of other colleagues who gave me useful feedback on the original article. Jiannis Seiradakis urged me to include the tables of parameters given in the appendix after I promised to put them in the original article, but didn't. The tables and references should be useful to both observers and theorists. Thanks also to Fernando Camilo for allowing me to include details on a number of pulsars in these tables prior to publication.

I am indebted to a number of colleagues who kindly gave permission to use a selection of figures in this article. Michael Kramer provided the cute animation of the rotating neutron star presented in Fig. 2. Vicky Kalogera provided the graph used in Fig. 15. Joe Taylor and Joel Weisberg provided the updated orbital decay curve of PSR B1913+16 shown in Fig. 23. Fig. 20, based on unpublished timing observations carried out at Jodrell Bank, was supplied by Andrew Lyne. Vicky Kaspi provided the millisecond pulsar timing residuals and comparison of their timing stabilities shown in Figs. 25 and 21 respectively. Andrea Lommen provided the pulsar timing residuals from the Arecibo timing program used to produce Fig. 27. Frequent use was made of NASA's magnificent Astrophysics Data System [172] and LANL's preprint archives [137] during the literature searches. Finally, I'd like to thank the Living Reviews editor, Theresa Velden, for being extraordinarily patient, and for putting up with many a feeble excuse from me during the writing of this long-overdue update. 


\section{Appendix: Tables of Binary and Millisecond Pulsars}

\begin{tabular}{lrrrrrr}
\hline \hline Name & $\begin{array}{c}P \\
{[\mathrm{~ms}]}\end{array}$ & $\log \tau_{\mathrm{c}}$ & $\log B$ & $\begin{array}{c}d \\
{[\mathrm{kpc}]}\end{array}$ & $\begin{array}{c}v_{\mathrm{t}} \\
{[\mathrm{km} / \mathrm{s}]}\end{array}$ & \multicolumn{2}{c}{ Ref. } \\
\hline J0030+0451 & 4.865 & 9.9 & 8.3 & 0.23 & $<65$ & {$[127]$} \\
$\mathrm{J} 0711-6830$ & 5.491 & 10.4 & 8.2 & 1.04 & 139 & {$[20,250]$} \\
$\mathrm{J} 1024-0719$ & 5.162 & 9.7 & 8.5 & 0.35 & 45 & {$[20,250]$} \\
& & & & & & \\
$\mathrm{J} 1730-2304$ & 8.123 & 9.9 & 8.6 & 0.51 & 53 & {$[135,250]$} \\
$\mathrm{J} 1744-1134$ & 4.075 & 9.9 & 8.3 & 0.17 & 20 & {$[20,250]$} \\
$\mathrm{B} 1937+21$ & 1.558 & 8.4 & 8.6 & 9.65 & 22 & {$[17,117]$} \\
$\mathrm{J} 2124-3358$ & 4.931 & 9.9 & 8.4 & 0.25 & 67 & {$[20,250]$} \\
$\mathrm{J} 2235+1506$ & 59.767 & 10.0 & 9.4 & 1.15 & 98 & {$[51]$} \\
$\mathrm{J} 2322+2057$ & 4.808 & 10.2 & 8.2 & 0.78 & 89 & {$[182,52]$} \\
\hline \hline
\end{tabular}

Table 2: Parameters for the 9 isolated millisecond pulsars currently known in the Galactic disk. Listed are the spin period $P$, the base-10 logarithms of the characteric age $\tau_{\mathrm{c}}$ and surface magnetic field strength B (§ 2.4.1), the distance $d$ derived from the Taylor $\&$ Cordes electron density model [235] or independently (when available), and the transverse speed $v_{\mathrm{t}}$ inferred from $d$ and a proper motion measurement (when available). Key publications for each pulsar are referenced to the bibliography.

Living Reviews in Relativity (2001-5)

http://www. livingreviews .org 


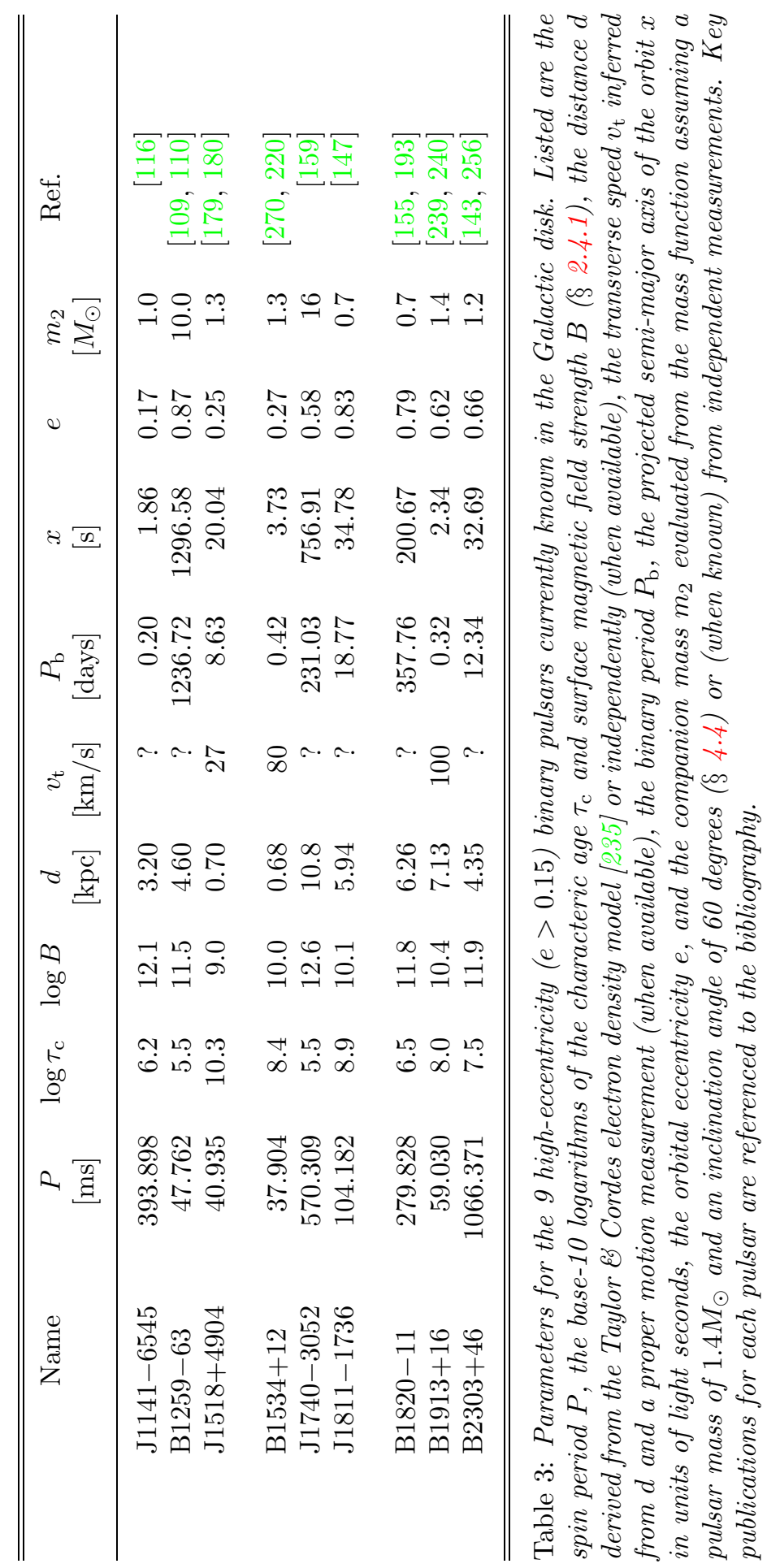

Living Reviews in Relativity (2001-5) http://www.livingreviews.org 


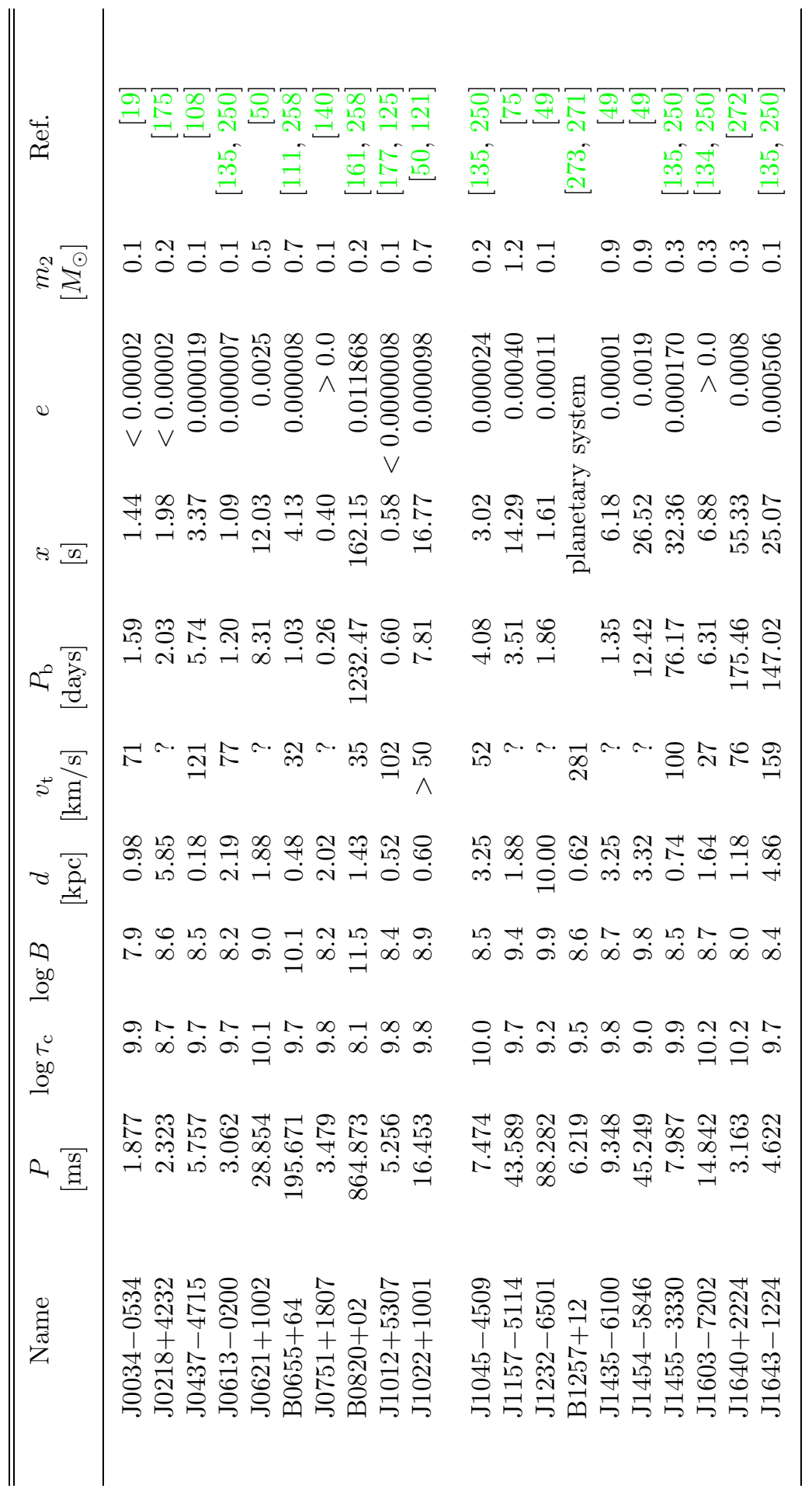

Living Reviews in Relativity (2001-5) http://www. livingreviews.org 


\begin{tabular}{|c|c|}
\hline 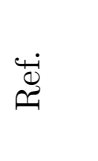 & 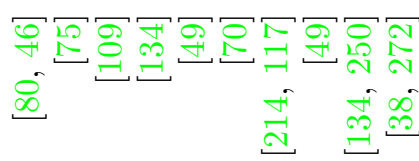 \\
\hline 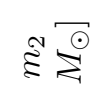 & 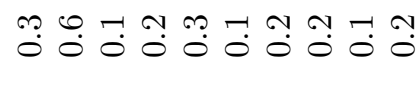 \\
\hline 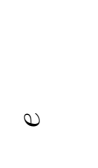 & 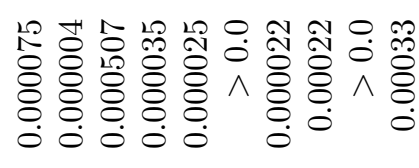 \\
\hline$\therefore \sqrt{n}$ & 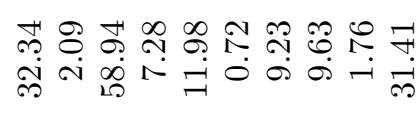 \\
\hline $2^{2} \frac{\sqrt[n]{0}}{0}$ & 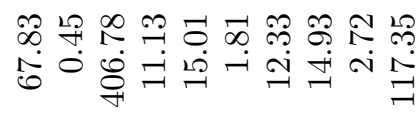 \\
\hline एक & 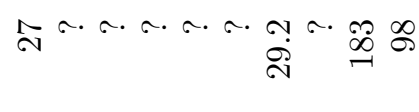 \\
\hline$\checkmark \underset{\tilde{q}}{\tilde{q}}$ & 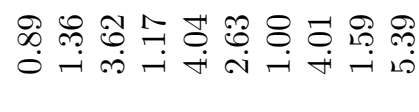 \\
\hline $\begin{array}{l}\infty \\
\infty \\
0\end{array}$ & 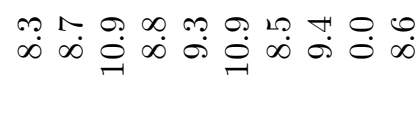 \\
\hline $\begin{array}{l}0 \\
.0 \\
0\end{array}$ & 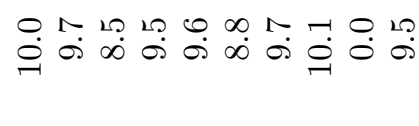 \\
\hline $2, \bar{g}$ & 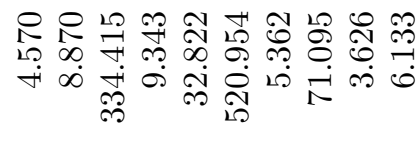 \\
\hline 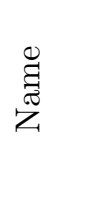 & 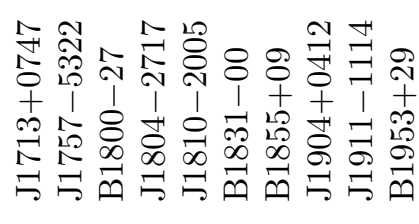 \\
\hline & 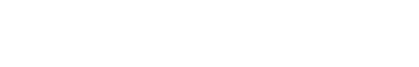 \\
\hline
\end{tabular}




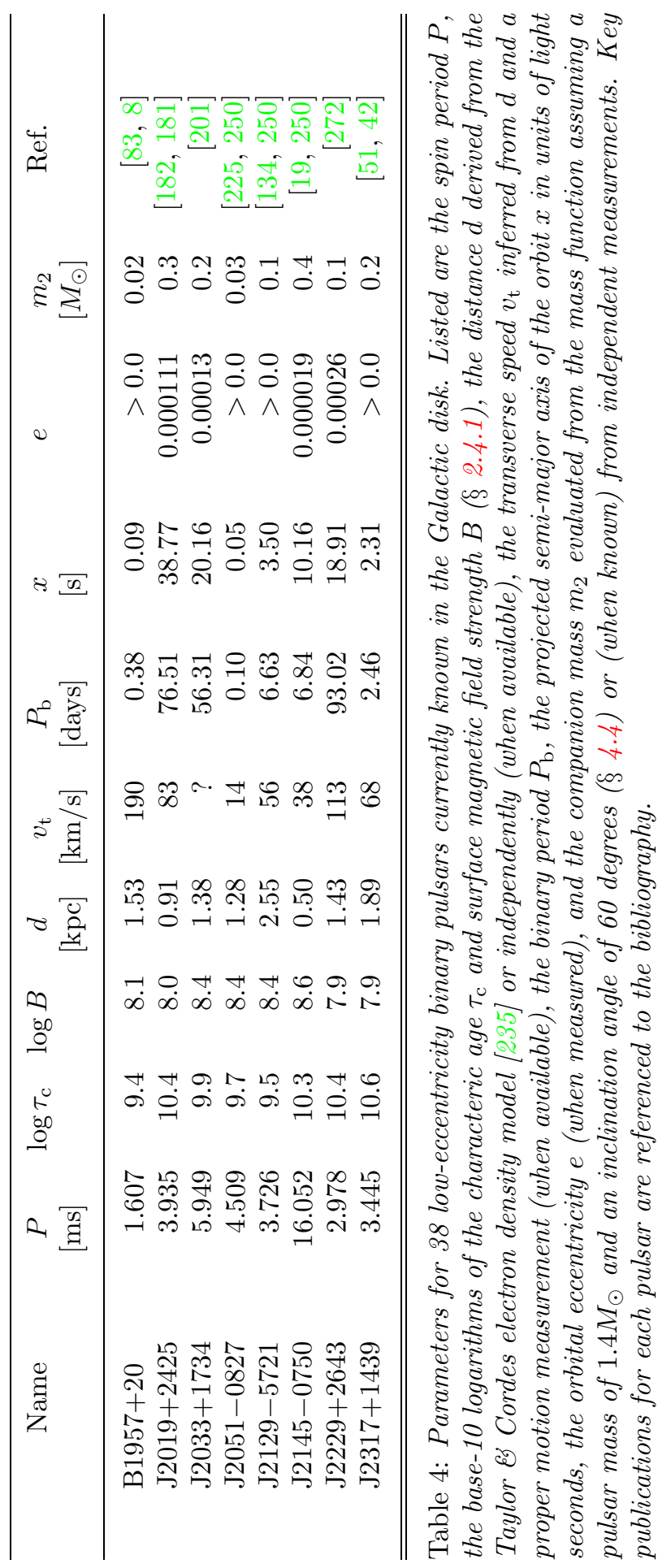

Living Reviews in Relativity (2001-5)

http://www. livingreviews .org 


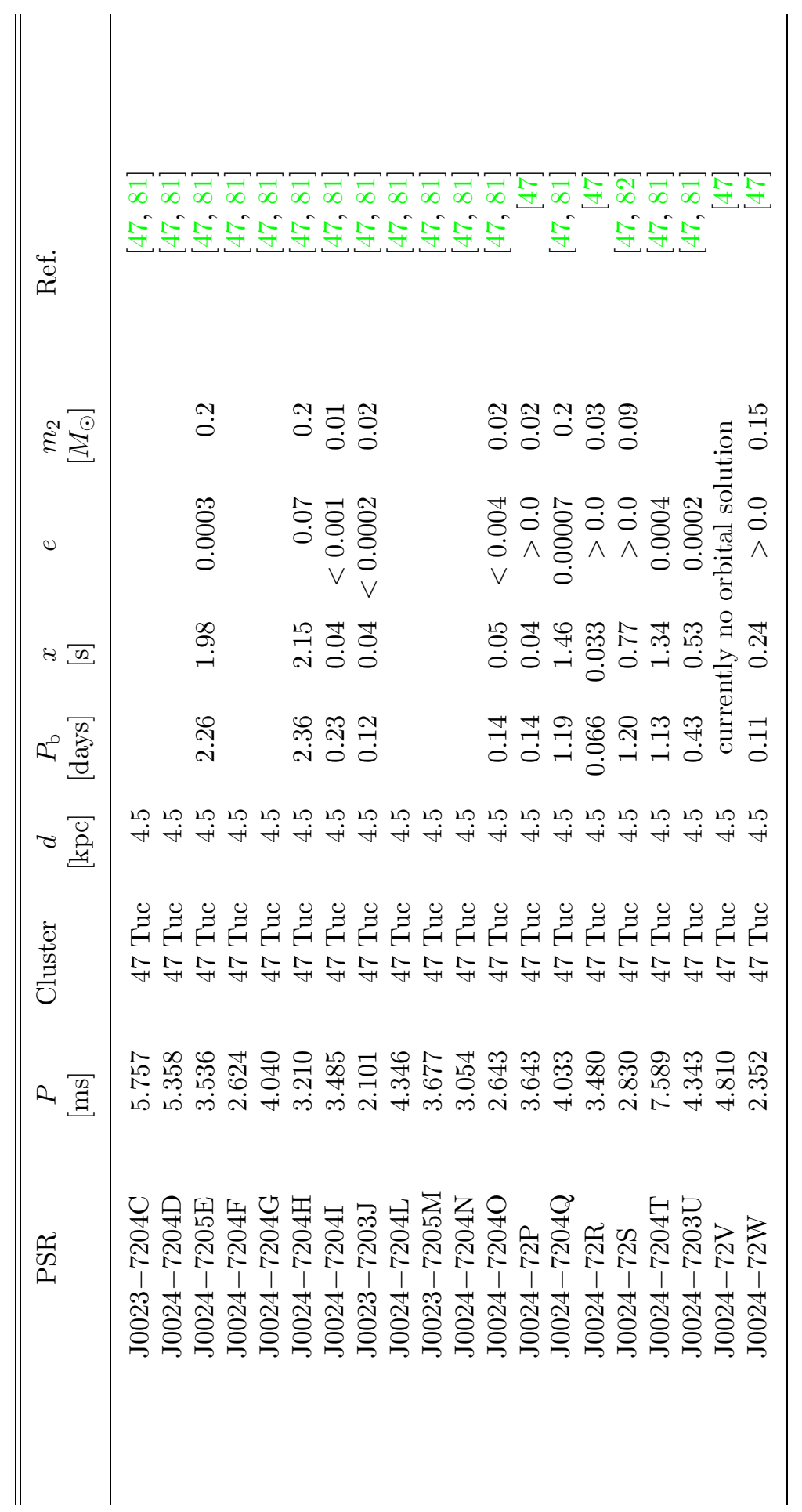

Living Reviews in Relativity (2001-5) http://www.livingreviews.org 


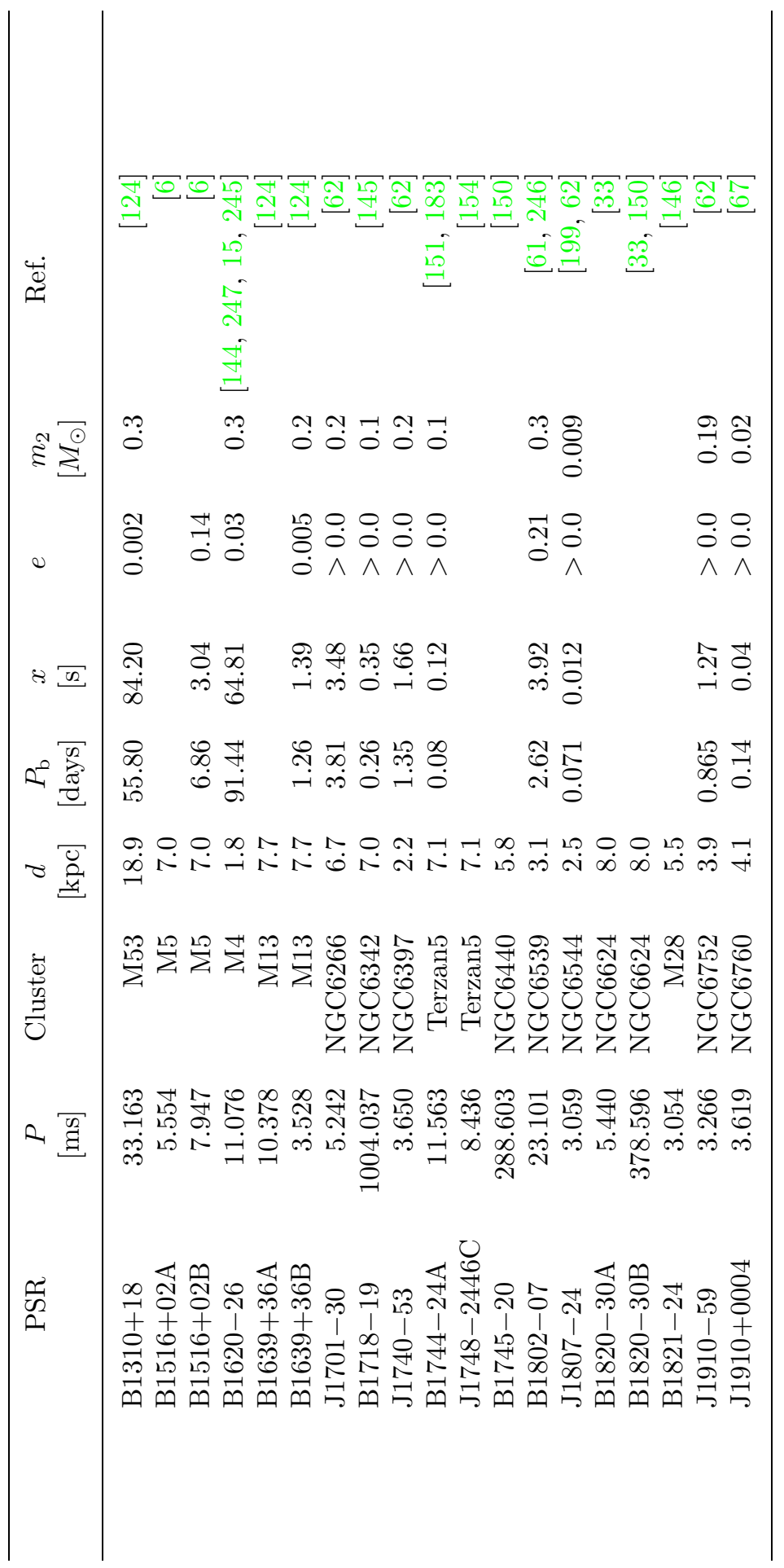

Living Reviews in Relativity (2001-5)

http://www. livingreviews .org 


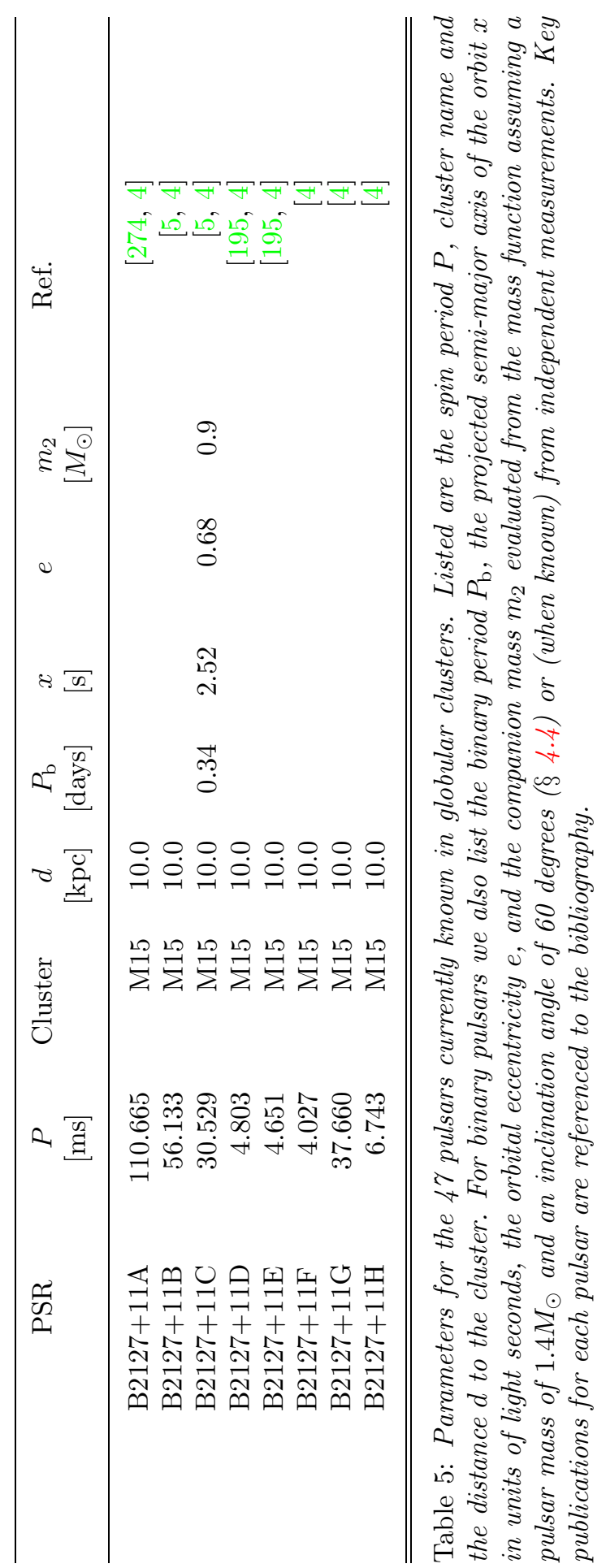

Living Reviews in Relativity (2001-5) http://www.livingreviews.org 


\section{References}

[1] Allan, D.W., "The Allan Variance", (December, 2000), [Online Resources]: cited on 7 December 2000, http://www.allanstime.com/ AllanVariance/. 4.3

[2] Alpar, M.A., Cheng, A.F., Ruderman, M.A., and Shaham, J., "A new class of radio pulsars", Nature, $\mathbf{3 0 0}, 728-730$, (1982). 2.4.3, 2.4.3

[3] Alpar, M.A., Nandkumar, R., and Pines, D., "Vortex Creep and the Internal Temperature of Neutron Stars: Timing Noise of Pulsars", Astrophys. J., 311, 197-213, (1986). 4.3

[4] Anderson, S.B., A study of recycled pulsars in globular clusters, PhD Thesis, (California Institute of Technology, Pasadena, Ca., USA, 1992). 7

[5] Anderson, S.B., Gorham, P.W., Kulkarni, S.R., Prince, T.A., and Wolszczan, A., "Discovery of Two Radio Pulsars in the Globular Cluster M15", Nature, 346, 42-44, (1990). 3.1.3, 7

[6] Anderson, S.B., Wolszczan, A., Kulkarni, S.R., and Prince, T.A., "Observations of Two Millisecond Pulsars in the Globular Cluster NGC 5904", Astrophys. J., 482, 870-873, (1997). 7

[7] Arzoumanian, Z., Cordes, J.M., and Wasserman, I., "Pulsar Spin Evolution, Kinematics, and the Birthrate of Neutron Star Binaries", Astrophys. J., 520, 696-705, (1999). 3.4 .1

[8] Arzoumanian, Z., Fruchter, A.S., and Taylor, J.H., "Orbital Variability in the Eclipsing Pulsar Binary PSR B1957+20", Astrophys. J., 426, L85L88, (1994). 7

[9] Arzoumanian, Z., Phillips, J.A., Taylor, J.H., and Wolszczan, A., "Radio Beam of the Relativistic Binary Pulsar B1534+12", Astrophys. J., 470, 1111-1117, (1996). 3.4.1, 4.6

[10] Backer, D. C., "Pulsars at Berkeley - Home Page", (March, 2001), [Online HTML Document]: cited on 20 March 2001, http://astro.berkeley. edu/ ${ }^{\text {mpulsar/. }} \quad 2.7,4.7,5.2$

[11] Backer, D.C., "Pulsars - Nature's Best Clocks", (October, 1999), [Audio and slides in jpg format]: cited on 8 December 2000, http://www. apscenttalks.org/pres_masterpage.cfm?nameID=65. 4.7

[12] Backer, D.C., "Timing of Millisecond Pulsars", in van Paradijs, J., van del Heuvel, E.P.J., and Kuulkers, E., eds., Compact Stars in Binaries: IAU Symposium 165, 197-211, (Kluwer, Dordrecht, 1996). 5.3

[13] Backer, D.C., Clifton, T.R., Kulkarni, S.R., and Wertheimer, D.J., "A digital signal processor for pulsar research", Astron. Astrophys., 232, 292300, (1990). 2.5

Living Reviews in Relativity (2001-5)

http://www. livingreviews.org 
[14] Backer, D.C., Dexter, M.R., Zepka, A., Ng, D., Werthimer, D.J., Ray, P.S., and Foster, R.S., "A Programmable $36 \mathrm{MHz}$ Digital Filter Bank for Radio Science", Publ. Astron. Soc. Pac., 109, 61-68, (1997). 4.1

[15] Backer, D.C., Foster, R.F., and Sallmen, S., "A second companion of the millisecond pulsar 1620-26", Nature, 365, 817-819, (1993). 2.4.2, 4.7, 7

[16] Backer, D.C., and Hellings, R.W., "Pulsar Timing and General Relativity", Annu. Rev. Astron. Astrophys., 24, 537-575, (1986). 4.2, 4.7

[17] Backer, D.C., Kulkarni, S.R., Heiles, C., Davis, M.M., and Goss, W.M., "A Millisecond Pulsar", Nature, 300, 615-618, (1982). 2.4.1, 4, 7

[18] Bailes, M., "The Origin of pulsar velocities and the velocity-magnetic moment correlation.", Astrophys. J., 342, 917-927, (1989). 2.4 .3

[19] Bailes, M., Harrison, P.A., Lorimer, D.R., Johnston, S., Lyne, A.G., Manchester, R.N., d'Amico, N., Nicastro, L., Tauris, T.M., and Robinson, C., "Discovery of Three Galactic Binary Millisecond Pulsars", Astrophys. J., 425, L41-L44, (1994). 7

[20] Bailes, M., Johnston, S., Bell, J.F., Lorimer, D.R., Stappers, B.W., Manchester, R.N., Lyne, A.G., d'Amico, N., and Gaensler, B.M., "Discovery of four isolated millisecond pulsars", Astrophys. J., 481, 386-391, (1997). 4, 2.4.3, 7

[21] Bailes, M., Manchester, R.N., Kesteven, M.J., Norris, R.P., and Reynolds, J.E., "The proper motion of six southern radio pulsars", Mon. Not. R. Astron. Soc., 247, 322-326, (1990). 2.4 .4

[22] Barker, B.M., and O'Connell, R.F., "Relativistic effects in the binary pulsar PSR 1913+16", Astrophys. J., 199, L25-L26, (1975). 4.6, 4.6

[23] Bell, J.F., "Radio Pulsar Timing", volume 21(1-2) of Adv. Space Res., 131-147. Elsevier Science, (1998). 4.7

[24] Bell, J.F., "Tests of Relativistic Gravity using Millisecond Pulsars", in Arzoumanian, Z., van der Hooft, F., and van den Heuvel, E.P.J., eds., Pulsar Timing, General Relativity, and the Internal Structure of Neutron Stars. North Holland, (1999). 4.7

[25] Bell, J.F., Bailes, M., and Bessell, M.S., "Optical detection of the companion of the millisecond pulsar PSR J0437-4715", Nature, 364, 603-605, (1993). $\quad 2.4 .3$

[26] Bell, J.F., Bailes, M., Manchester, R.N., Lyne, A.G., Camilo, F., and Sandhu, J.S., "Timing measurements and their implications for four binary millisecond pulsars", Mon. Not. R. Astron. Soc., 286, 463-469, (1997). 4 
[27] Bell, J.F., Kulkarni, S.R., Bailes, M., Leitch, E.M., and Lyne, A.G., "Optical Observations of the Binary Millisecond Pulsars J2145-0750 and J00340534", Astrophys. J., 452, L121-L124, (1995). 2.4 .3

[28] Bell, J.F., Manchester, R.N., Crawford, F., Lyne, A.G., Camilo, F., Kaspi, V.M., Stairs, I.H., Morris, D.J., d'Amico, N., McKay, N.P.F., Kramer, M., Sheppard, D.C., and Possenti, A., "The Parkes Multibram Pulsar Survey Data Release", in Kramer, M., Wex, N., and Wielebinski, R., eds., Pulsar Astronomy - 2000 and Beyond, Astronomical Society of the Pacific Conference Series, 9-10. Astronomical Society of the Pacific, (2000). For a related online version see: J.F. Bell, et al., "The Parkes Multibram Pulsar Survey Data Release", (November, 1999), [Online Los Alamos Preprint]: cited on 19 March 2001, http://arxiv.org/abs/astro-ph/9911321. To be published in APS Conference Series. 2.4.1

[29] Bertotti, B., Carr, B.J., and Rees, M.J., "Limits from the timing of pulsars on the cosmic gravitational wave background", Mon. Not. R. Astron. Soc., 203, 945-954, (1983). 5, 5.1, 5.1

[30] Beskin, V.S., Gurevich, A.V., and Istomin, Ya.N., Physics of the Pulsar Magnetosphere, (Cambridge University Press, 1993). 2.7

[31] Bhattacharya, D., and van den Heuvel, E.P.J., "Formation and evolution of binary and millisecond radio pulsars", Phys. Rep., 203, 1-124, (1991). 2.4.3, $2.7,3.5$

[32] Biggs, J.D., "Meridional compression of radio pulsar beams", Mon. Not. R. Astron. Soc., 245, 514-521, (1990). 3.2.3, 16, 3.3 .1

[33] Biggs, J.D., Bailes, M., Lyne, A.G., Goss, W.M., and Fruchter, A.S., "Two radio pulsars in the globular cluster NGC 6624", Mon. Not. R. Astron. Soc., 267, 125-128, (1994). 7

[34] Bisnovatyi-Kogan, G.S., and Komberg, B.V., "Pulsars and Close Binary Systems", Sov. Astron., 18, 217-221, (1974). 2.4 .3

[35] Blaauw, A., "On the origin of the O- and B-type stars with high velocities (the 'Run-away' stars), and related problems", Bull. Astron. Inst. Neth., 15, 265-290, (1961). 2.4 .3

[36] Blandford, R.D., Narayan, R., and Romani, R.W., "Arrival-time analysis for a millisecond pulsar", J. Astrophys. Astron., 5, 369-388, (1984). 5, 5.1

[37] Blandford, R.D., and Teukolsky, S.A., "Arrival-time analysis for a pulsar in a binary system", Astrophys. J., 205, 580-591, (1976). 4.5

[38] Boriakoff, V., Buccheri, R., and Fauci, F., "Discovery of a 6.1-ms Binary Pulsar, PSR 1953+29", Nature, 304, 417-419, (1983). 7

Living Reviews in Relativity (2001-5)

http://www.livingreviews.org 
[39] Burderi, L., and d'Amico, N., "Probing the Equation of State of Ultradense matter with a Submillisecond Pulsar Search Experiment", Astrophys. J., 490, 343-352, (1997). 6

[40] Caldwell, R.R., and Allen, B., "Cosmological constraints on cosmic-string gravitational radiation", Phys. Rev. D, 45, 3447-3468, (1992). 5.1

[41] Camilo, F., "Millisecond Pulsar Searches", in Alpar, A., Kiziloğlu, Ü., and van Paradis J., eds., The Lives of the Neutron Stars (NATO ASI Series), 243-257, (Kluwer, Dordrecht, 1995). 2.6.1

[42] Camilo, F., A Search for Millisecond Pulsars, PhD Thesis, (Princeton University, Princeton, 1995). 7

[43] Camilo, F., "Intermediate-mass binary pulsars: a new class of objects?", in Johnston, S., Walker, M.A., and Bailes, M., eds., Pulsars: Problems and Progress, IAU Colloquium 160, 539, (Astronomical Society of the Pacific, San Francisco, 1996). 2.4.3

[44] Camilo, F., "Present and future pulsar searches", in Jackson, N., and Davis, R.J., eds., High Sensitivity Radio Astronomy, 14-22. Cambridge University Press, (1997). 2.6.1

[45] Camilo, F., "Pulsar Searches in the Northern Hemisphere", in Arzoumanian, Z., van der Hooft, F., and van den Heuvel, E.P.J., eds., Pulsar Timing, General Relativity, and the Internal Structure of Neutron Stars, 115, (North Holland, Amsterdam, 1999). 2.6.1

[46] Camilo, F., Foster, R.S., and Wolszczan, A., "High-Precision Timing of PSR J1713+0747: Shapiro Delay", Astrophys. J., 437, L39-L42, (1994). $4.2,4.5,7$

[47] Camilo, F., Lorimer, D.R., Freire, P., Lyne, A.G., and Manchester, R.N., "Observations of 20 millisecond pulsars in 47 Tucanae at $20 \mathrm{~cm}$ ", Astrophys. J., 535, 975-990, (2000). 2.6.4, 3.1.3, 6, 7

[48] Camilo, F., Lyne, A.G., Manchester, R.N., Bell, J.F., Kaspi, V.M., d'Amico, N., McKay, N.P.F., Crawford, F., Stairs, I.H., , Morris, D.J., Sheppard, D.C., and Possenti, A., "The Parkes Multibram Pulsar Survey", in Kramer, M., Wex, N., and Wielebinski, R., eds., Pulsar Astronomy - 2000 and Beyond, Astronomical Society of the Pacific Conference Series, 3-8. Astronomical Society of the Pacific, (2000). 2.6.2

[49] Camilo, F., Lyne, A.G., Manchester, R.N., Bell, J.F., Stairs, I.H., d'Amico, N., Kaspi, V.M., Possenti, A., Crawrd, F., and McKay, N.P.F., "Discovery of five binary radio pulsars", Astrophys. J., 548, L187-L191, (2001). 2.4.3, 2.6.2, 7 
[50] Camilo, F., Nice, D.J., Shrauner, J.A., and Taylor, J.H., "PrincetonArecibo all-sky survey for millisecond pulsars. I", Astrophys. J., 469, 819-827, (1996). 7

[51] Camilo, F., Nice, D.J., and Taylor, J.H., "Discovery of two fast-rotating pulsars", Astrophys. J., 412, L37-L40, (1993). 7, 7

[52] Camilo, F., Nice, D.J., and Taylor, J.H., "A search for millisecond pulsars at galactic latitudes $-50^{\circ}<b<-20^{\circ}$ ", Astrophys. J., 461, 812-819, (1996). 7

[53] Chakrabarty, D., and Morgan, E.H., "The 2 hour orbit of a binary millisecond X-ray pulsar", Nature, 394, 346-348, (1998). 2.4 .3

[54] Cheng, K.S., "Could Glitches Inducing Magnetospheric Fluctuations Produce Low Frequency Timing Noise?", Astrophys. J., 321, 805-812, (1987). 4.3

[55] Cheng, K.S., "Outer Magnetospheric Fluctuations and Pulsar Timing Noise", Astrophys. J., 321, 799-804, (1987). 4.3

[56] Clifton, T.R., Lyne, A.G., Jones, A.W., McKenna, J., and Ashworth, M., "A high frequency survey of the Galactic plane for young and distant pulsars", Mon. Not. R. Astron. Soc., 254, 177-184, (1992). 3.1 .2

[57] Cordes, J.M., and Chernoff, D.F., "Neutron Star Population Dynamics. I. Millisecond Pulsars", Astrophys. J., 482, 971-992, (1997). 2.4.4

[58] Cordes, J.M., and Chernoff, D.F., "Neutron Star Population Dynamics. II. Three-Dimensional Space Velocities of Young Pulsars", Astrophys. J., 505, 315-338, (1998). $\quad 2.4 .4$

[59] Cordes, J.M., and Helfand, D.J., "Pulsar timing III. Timing Noise of 50 Pulsars", Astrophys. J., 239, 640-650, (1980). 4.3

[60] Curran, S.J., and Lorimer, D.R., "Pulsar statistics III: Neutron star binaries.", Mon. Not. R. Astron. Soc., 276, 347-352, (1995). 3.4 .1

[61] d'Amico, N., Bailes, M., Lyne, A.G., Manchester, R.N., Johnston, S., Fruchter, A.S., and Goss, W.M., "PSR B1802-07: A globular cluster pulsar in an eccentric binary system", Mon. Not. R. Astron. Soc., 260, L7L10, (1993). 7

[62] d'Amico, N., Lyne, A.G., Manchester, R.N., Possenti, A., and Camilo, F., "Discovery of short-period binary millisecond pulsars in four globular clusters", Astrophys. J., 548, L171-L174, (2001). 2.6.4, 6, 7

[63] Damour, T., and Ruffini, R., "On certain new verifications of general relativity made possible by the discovery of a pulsar member of a binary system", Comptes Rendus Acad. Sci. Ser. A, 279, 971-973, (1974). 4.6

Living Reviews in Relativity (2001-5)

http://www.livingreviews.org 
[64] Dana, P.H., "The Global Positioning System", (May, 2000), [Online HTML Document]: cited on 19 March 2001, http://www.Colorado. EDU/geography/gcraft/notes/gps/gps_f.html. 4.1

[65] Davis, M.M., Taylor, J.H., Weisberg, J.M., and Backer, D.C., "Highprecision timing Observations of the Millisecond pulsar PSR 1937+21", Nature, 315, 547-550, (1985). 4

[66] Deich, W.T.S., and Kulkarni, S.R., "The Masses of the Neutron Stars in M15C", in van Paradijs, J., van del Heuvel, E.P.J., and Kuulkers, E., eds., Compact Stars in Binaries: IAU Symposium 165, 279-285, (Kluwer, Dordrecht, 1996). $\quad 4.5$

[67] Deich, W.T.S., Middleditch, J., Anderson, S.B., Kulkarni, S.R., Prince, T.A., and Wolszczan, A., "The binary pulsar PSR 1908+00 in NGC 6760", Astrophys. J., 410, L95-L98, (1993). 7

[68] Detweiler, S., "Pulsar timing measurements and the search for gravitational waves", Astrophys. J., 234, 1100-1104, (1979). 5, 5.1

[69] Dewey, R.J., and Cordes, J.M., "Monte Carlo Simulations of Radio Pulsars and their Progenitors", Astrophys. J., 321, 780-798, (1987). 3.5

[70] Dewey, R.J., Maguire, C.M., Rawley, L.A., Stokes, G.H., and Taylor, J.H., "Binary pulsar with a very small mass function", Nature, 322, 712-714, (1986). 7

[71] Dewey, R.J., Stokes, G.H., Segelstein, D.J., Taylor, J.H., and Weisberg, J.M., "The Period Distribution of Pulsars", in Reynolds, S.P., and Stinebring, D.R., eds., Millisecond Pulsars, 234-240. NRAO : Green Bank, (1984). 2

[72] Doroshenko, O.V., and Kopeikin, S.M., "Relativistic effect of graviational deflection of light in binary pulsars", Mon. Not. R. Astron. Soc., 274, 1029, (1995). 4.7

[73] Downs, G.S., and Reichley, P.E., "JPL pulsar timing observations. II. Geocentric arrival times.", Astrophys. J. Suppl. Ser., 53, 169-240, (1983). 5.1

[74] Edwards, R., "Discovery of Eight Recycled Pulsars - The Swinburne Intermediate Latitude Pulsar Survey", in Kramer, M., Wex, N., and Wielebinski, R., eds., Pulsar Astronomy - 2000 and Beyond, Astronomical Society of the Pacific Conference Series, 33-34. Astronomical Society of the Pacific, (2000). 2.6.3

[75] Edwards, R., and Bailes, M., "Discovery of two relativistic neutron starwhite dwarf binaries", Astrophys. J., 547, L37-L40, (2000). 2.6.3, 3.4.2, 7 
[76] Esposito, L.W., and Harrison, E.R., "Properties of the Hulse-Taylor binary pulsar system", Astrophys. J., 196, L1-L2, (1975). 4.6

[77] Flannery, B.P., and van den Heuvel, E.P.J., "On the origin of the binary pulsar PSR 1913+16", Astron. Astrophys., 39, 61-67, (1975). 2.4 .3

[78] Fomalont, E.B., Goss, W.M., Lyne, A.G., Manchester, R.N., and Justtanont, K., "Positions and Proper Motions of Pulsars", Mon. Not. R. Astron. Soc., 258, 497-510, (1992). 2.4 .4

[79] Foster, R.S., and Backer, D.C., "Constructing a Pulsar Timing Array", Astrophys. J., 361, 300, (1990). 5.2

[80] Foster, R.S., Wolszczan, A., and Camilo, F., "A new binary millisecond pulsar", Astrophys. J., 410, L91-L94, (1993). 7

[81] Freire, P., Camilo, F., Lorimer, D.R., Lyne, A.G., Manchester, R.N., and d'Amico, N., "Timing the millisecond pulsars in 47 Tucanae", Mon. Not. R. Astron. Soc., (2000). In press. 2.6.4, 7

[82] Freire, P., Kramer, M., and Lyne, A.G., "Determination of the orbital parameters of binary pulsars", (October, 2000), [Online Los Alamos Preprint]: cited on 19 March 2001, http://arxiv.org/abs/astro-ph/ 0010463. Mon. Not. R. Astron. Soc., in press (2001). 7

[83] Fruchter, A.S., Stinebring, D.R., and Taylor, J.H., "A millisecond pulsar in an eclipsing binary", Nature, 333, 237-239, (1988). 2.4.3, 7

[84] Fryer, C., Burrows, A., and Benz, W., "Population Syntheses for Neutron Star Systems with Intrinsic Kicks", Astrophys. J., 496, 333, (1998). 2.4 .4

[85] Gold, T., "Rotating Neutron Stars as the Origin of the Pulsating Radio Sources", Nature, 218, 731-732, (1968). 2.1

[86] Gold, T., "Rotating neutron stars and the nature of pulsars", Nature, 221, 25-27, (1969). 2.1

[87] Gould, D.M., and Lyne, A.G., "Multifrequency polarimetry of 300 radio pulsars", Mon. Not. R. Astron. Soc., 301, 235-260, (1998). 4

[88] Gunn, J.E., and Ostriker, J.P., "On the nature of pulsars. III. Analysis of observations.", Astrophys. J., 160, 979-1002, (1970). 3

[89] Han, J.L., and Manchester, R.N., "The shape of radio pulsar beams", Mon. Not. R. Astron. Soc., 320, L35-L40, (2001). For a related online version see: J.L. Han, et al., "The shape of radio pulsar beams", (October, 2000), [Online Los Alamos Preprint]: cited on 19 March 2001, http: //arxiv.org/abs/astro-ph/0010538. 1

[90] Hankins, T.H., "Microsecond Intensity Variation in the Radio Emission from CP 0950", Astrophys. J., 169, 487-494, (1971). 4.1 
[91] Hansen, B., and Phinney, E.S., "The pulsar kick velocity distribution", Mon. Not. R. Astron. Soc., 291, 569-577, (1997). 2.4 .4

[92] Harrison, P.A., Lyne, A.G., and Anderson, B., "New Determinations of the Proper Motions of 44 Pulsars", Mon. Not. R. Astron. Soc., 261, 113124, (1993). 2.4.4

[93] Hartman, J.W., "On the velocity distribution of radio pulsars at birth", Astron. Astrophys., 322, 127-130, (1997). 2.4 .4

[94] Helfand, D.J., Manchester, R.N., and Taylor, J.H., "Observations of pulsar radio emission. III. Stability of integrated profiles.", Astrophys. J., 198, 661-670, (1975). 1

[95] Hellings, R.W., "Thoughts on Detecting a Gravitational Wave Background with Pulsar Data", in Backer, D., ed., Impact of Pulsar Timing on Relativity and Cosmology, k1, (Center for Particle Astrophysics, Berkeley, 1990). 5.2

[96] Hellings, R.W., and Downs, G.S., "Upper Limits on the Isotropic Gravitational Radiation Background from Pulsar Timing Analysis", Astrophys. J., 265, L39, (1983). 5.2

[97] Hewish, A., Bell, S.J., lkington, J.D.H., Scott, P.F., and Collins, R.A., "Observation of a Rapidly Pulsating Radio Source", Nature, 217, 709713, (1968). 1, 2, 4

[98] Hills, J.G., "The effects of Sudden Mass loss and a random kick velocity produced in a supernova explosion on the Dynamics of a binary star of an Arbitrary orbital Eccentricity", Astrophys. J., 267, 322-333, (1983). 2.4 .3

[99] Hogan, C.J., and Rees, M.J., "Gravitational interactions of cosmic strings", Nature, 311, 109-114, (1984). 5.1

[100] Hough, J., and Rowan, S., "Interferometric Gravitational Wave Detectors (Ground and Space)", (June, 2000), [Article in Online Journal Living Reviews in Relativity]: cited on 19 March 2001, http: //www.livingreviews.org/Articles/Volume3/2000-3hough. 3.4

[101] Hulse, R.A., "The discovery of the binary pulsar", Rev. Mod. Phys., 66, 699-710, (1994). 4.5

[102] Hulse, R.A., and Taylor, J.H., "Discovery of a pulsar in a binary system", Astrophys. J., 195, L51-L53, (1975). 2.4.3, 3.1.3, 3.4.1

[103] Hunt, G.C., "The rate of change of period of the pulsars", Mon. Not. R. Astron. Soc., 153, 119-131, (1971). 4.2 
[104] Jimenez, R., Flynn, C., and Kotoneva, E., "HIPPARCOS and the age of the Galactic disc", Mon. Not. R. Astron. Soc., 299, 515-519, (1998). 3.3.2

[105] Jodrell Bank Pulsar Page, "Pulsars at Jodrell Bank", (March, 2001), [Online HTML Document]: cited on 20 March 2001, http://www.jb.man. ac.uk/ ${ }^{\sim}$ pulsar/. 2.7

[106] Johnston, H.M., and Kulkarni, S.R., "On the detectability of pulsars in close binary systems", Astrophys. J., 368, 504-514, (1991). 3.1 .3

[107] Johnston, S., "Evidence for a deficit of pulsars in the inner Galaxy", Mon. Not. R. Astron. Soc., 268, 595-601, (1994). 3.3 .2

[108] Johnston, S., Lorimer, D.R., Harrison, P.A., Bailes, M., Lyne, A.G., Bell, J.F., Kaspi, V.M., Manchester, R.N., d'Amico, N., Nicastro, L., and Jin, Shengzhen, "Discovery of a very bright, nearby binary millisecond pulsar", Nature, 361, 613-615, (1993). 3.3.3, 7

[109] Johnston, S., Lyne, A.G., Manchester, R.N., Kniffen, D.A., d'Amico, N., Lim, J., and Ashworth, M., "A High Frequency Survey of the Southern Galactic Plane for Pulsars", Mon. Not. R. Astron. Soc., 255, 401-411, (1992). 3.1.2, 4.6, 7, 7

[110] Johnston, S., Manchester, R.N., Lyne, A.G., Bailes, M., Kaspi, V.M., Qiao, Guojun, and d'Amico, N., "PSR 1259-63: A binary radio pulsar with a Be star companion", Astrophys. J., 387, L37-L41, (1992). 2.4.3, 7

[111] Jones, A.W., and Lyne, A.G., "Timing Observations of the Binary Pulsar PSR 0655+64", Mon. Not. R. Astron. Soc., 232, 473, (1988). 7

[112] Kalogera, V., "Close Binaries with Two Compact Objects", in Kramer, M., Wex, N., and Wielebinski, R., eds., Pulsar Astronomy - 2000 and Beyond, Astronomical Society of the Pacific Conference Series, 579-584. Astronomical Society of the Pacific, (2000). 3.2.2, 3.5

[113] Kalogera, V., Narayan, R., Spergel, D.N., and Taylor, J.H., "The Coalescence Rate of Double Neutron Star Systems", (December, 2000), [Online Los Alamos Preprint]: cited on 19 March 2001, http://arxiv.org/abs/ astro-ph/0012038. Submitted to Astrophys. J. 3.2.2, 3.4.1

[114] Kaspi, V.M., Applications of Pulsar Timing, PhD Thesis, (Princeton University, Princeton, 1994). 4.2

[115] Kaspi, V.M., Johnston, S., Bell, J.F., Manchester, R.N, Bailes, M., Bessell, M., Lyne, A.G., and d'Amico, N., "A Massive Radio Pulsar Binary in the Small Magellanic Cloud", Astrophys. J., 423, L43-L45, (1994). 2.4 .3

Living Reviews in Relativity (2001-5)

http://www.livingreviews.org 
[116] Kaspi, V.M., Lyne, A.G., Manchester, R.N., Crawford, F., Camilo, F., Bell, J.F., d'Amico, N., Stairs, I.H., McKay, N.P.F., Morris, D.J., and Possenti, A., "Discovery of a young radio pulsar in a relativistic binary orbit", Astrophys. J., 534, 321-327, (2000). 2.6.2, 3.4.2, 4.6, 7

[117] Kaspi, V.M., Taylor, J.H., and Ryba, M., "High-Precision Timing of Millisecond Pulsars. III. Long-Term Monitoring of PSRs B1855+09 and B1937+21", Astrophys. J., 428, 713-728, (1994). 4.2, 4.2, 4.3, 21, 4.3, $4.5,25,7,7$

[118] Kopeikin, S. M., "Binary Pulsars as Detectors of Ultra-Low Frequency Graviational Waves", Phys. Rev. D, 56, 4455, (1997). 5.1

[119] Kramer, M., "Determination of the Geometry of the PSR B1913+16 System by Geodetic Precession", Astrophys. J., 509, 856-860, (1998). 3.4.1, 4.6, 4.6, 24

[120] Kramer, M., "Evidence for Geodetic Precession in Binary Pulsars", in Gurzadyan, V., Jantzen, R., and Ruffini, R., eds., Proceedings of the 9th Marcel Grossman Meeting, (World Scientific, Singapore, 2001). Submitted. $4.6,4.7$

[121] Kramer, M., Xilouris, K.M., Camilo, F., Nice, D., Lange, C., Backer, D.C., and Doroshenko, O., "Profile instabilities of the millisecond pulsar J1022+1001", Astrophys. J., 520, 324-334, (1999). 7

[122] Kramer, M., Xilouris, K.M., Lorimer, D.R., Doroshenko, O., Jessner, A., Wielebinski, R., Wolszczan, A., and Camilo, F., "The characteristics of millisecond pulsar emission: I. Spectra, pulse shapes and the beaming fraction", Astrophys. J., 501, 270-285, (1998). 4, 2.4.3, 3.2.3, 3.3.1

[123] Kulkarni, S.R., and Anderson, S.B., "Pulsars in globular clusters", in Hut, P., and Makino, J., eds., Dynamical Evolution of Star Clusters Confrontation of Theory and Observations, volume 174 of Proceedings of the 174th Symposium of the IAU, 181-182, (Kluwer Academic Publishers, Dordrecht, 1996). 2.6.4

[124] Kulkarni, S.R., Anderson, S.B., Prince, T.A., and Wolszczan, A., "Old pulsars in the low-density globular clusters M13 and M53", Nature, 349, 47-49, (1991). 7

[125] Lange, C., Camilo, F., Wex, N., Kramer, M., Backer, D.C. Lyne, A.G., and Doroshenko, O., "Precision timing measurements of PSR J1012+5307", Mon. Not. R. Astron. Soc., (2001). Submitted. 22, 7

[126] Lawson, K.D., Mayer, C.J., Osborne, J.L., and Parkinson, M.L., "Variations in the Spectral Index of the Galactic Radio Continuum Emission in the Northern Hemisphere", Mon. Not. R. Astron. Soc., 225, 307-327, (1987). $\quad 3.1 .2$ 
[127] Lommen, A.N., Zepka, A., Backer, D.C., McLaughlin, M.A., Cordes, J.M., Arzoumanian, Z., and Xilouris, K., "New pulsars from an Arecibo drift scan search", Astrophys. J., 545, 1007, (2001). 7

[128] Lorimer, D.R., "Binary and Millisecond Pulsars", (March, 1998), [Article in Online Journal Living Reviews in Relativity]: cited on 19 March 2001, http://www.livingreviews.org/Articles/Volume1/ 1998-10lorimer. 1.1

[129] Lorimer, D.R., "Pulsar Statistics - II. The local low-mass binary pulsar population", Mon. Not. R. Astron. Soc., 274, 300-304, (1995). 2.4 .4

[130] Lorimer, D.R., "Pulsar Searches - Tricks of the Trade", in Davier, M., and Hello, P., eds., Second Workshop on Gravitational Wave Data Analysis, 121-132. Editions Frontiers, (1998). 2.5

[131] Lorimer, D.R., Bailes, M., Dewey, R.J., and Harrison, P.A., "Pulsar Statistics: The Birthrate and initial spin periods of radio pulsars", Mon. Not. R. Astron. Soc., 263, 403-415, (1993). 3.2 .1

[132] Lorimer, D.R., Bailes, M., and Harrison, P.A., "Pulsar Statistics IV: Pulsar Velocities", Mon. Not. R. Astron. Soc., 289, 592-604, (1997). 2.4 .4

[133] Lorimer, D.R., Festin, L., Lyne, A.G., and Nicastro, L., "Birth rate of millisecond pulsars", Nature, 376, 393, (1995). 2.4 .3

[134] Lorimer, D.R., Lyne, A.G., Bailes, M., Manchester, R.N., d'Amico, N., Stappers, B.W., Johnston, S., and Camilo, F., "Discovery of four binary millisecond pulsars", Mon. Not. R. Astron. Soc., 283, 1383-1387, (1996). 7

[135] Lorimer, D.R., Nicastro, L., Lyne, A.G., Bailes, M., Manchester, R.N., Johnston, S., Bell, J.F., d'Amico, N., and Harrison, P.A., "Four new millisecond pulsars in the Galactic disk", Astrophys. J., 439, 933-938, (1995). $\quad 3.3 .2,7,7$

[136] Lorimer, D.R.and Yates, J.A., Lyne, A.G., and Gould, D.M., "Multifrequency Flux density measurements of 280 pulsars", Mon. Not. R. Astron. Soc., 273, 411-421, (1995). 3.1 .2

[137] Los Alamos National Laboratories, "arXiv.org e-Print archive", [Online Database]: cited on March 19 2001, http://arxiv.org. 6.1

[138] Lundgren, S.C., Camilo, F., and Foster, R.S., "HST observations of millisecond pulsar companions: constraints on evolution", in Johnston, S., Walker, and M, M.A.. Bailes, eds., Pulsars: Problems and Progress, IAU Colloquium 160, 497-500, (Astronomical Society of the Pacific, San Francisco, 1996). $\quad 2.4 .3$

Living Reviews in Relativity (2001-5)

http://www.livingreviews.org 
[139] Lundgren, S.C., Cordes, J.M., Foster, R.S., Wolszczan, A., and Camilo, F., "Optical studies of millisecond pulsar companions", Astrophys. J., 458, L33-L36, (1996). 2.4.3

[140] Lundgren, S.C., Zepka, A.F., and Cordes, J.M., "A millisecond pulsar in a six hour orbit: PSR J0751+1807", Astrophys. J., 453, 419-423, (1995). 7

[141] Lyne, A.G., "Radio pulsar search techniques", in Schutz, B.F., ed., Gravitational Wave Data Analysis, (NATO ASI Series), 95-104, (Reidel, Dordrecht, 1988). 2.5

[142] Lyne, A.G., Anderson, B., and Salter, M.J., "The Proper Motions of 26 Pulsars", Mon. Not. R. Astron. Soc., 201, 503-520, (1982). 2.4 .4

[143] Lyne, A.G., and Bailes, M., "The mass of the PSR 2303+46 system", Mon. Not. R. Astron. Soc., 246, 15P-17P, (1990). 3.4.2, 7

[144] Lyne, A.G., Biggs, J.D., Brinklow, A., Ashworth, M., and McKenna, J., "Discovery of a binary millisecond pulsar in the globular cluster M4", Nature, 332, 45-47, (1988). 7

[145] Lyne, A.G., Biggs, J.D., Harrison, P.A., and Bailes, M., "A long-period globular-cluster pulsar in an eclipsing binary system", Nature, 361, 47-49, (1993). 7

[146] Lyne, A.G., Brinklow, A., Middleditch, J., Kulkarni, S.R., Backer, D.C., and Clifton, T.R., "The Discovery of a Millisecond Pulsar in the Globular Cluster M28", Nature, 328, 399-401, (1987). 7

[147] Lyne, A.G., Camilo, F., Manchester, R.N., Bell, J.F., Kaspi, V.M., d'Amico, N., McKay, N.P.F., Crawford, F., Morris, D.J., Sheppard, D.C., and Stairs, I.H., "The Parkes multibeam survey: PSR J1811-1736 - a pulsar in a highly eccentric binary system", Mon. Not. R. Astron. Soc., 312, 698-702, (2000). 2.6.2, 3.1.2, 22, 7

[148] Lyne, A.G., and Lorimer, D.R., "High birth velocities of radio pulsars", Nature, 369, 127-129, (1994). 2.4.4, 2.4.4

[149] Lyne, A.G., and Manchester, R.N., "The shape of pulsar radio beams", Mon. Not. R. Astron. Soc., 234, 477-508, (1988). 1, 3.2.3, 16

[150] Lyne, A.G., Manchester, R.N., and d'Amico, N., "PSR B1745-20 and young pulsars in globular clusters", Astrophys. J., 460, L41-L44, (1996). 7

[151] Lyne, A.G., Manchester, R.N., d'Amico, N., Staveley-Smith, L., Johnston, S., Lim, J., Fruchter, A.S., Goss, W.M., and Frail, D., "An Eclipsing Millisecond Pulsar in the Globular Cluster Terzan 5", Nature, 347, 650652, (1990). 7 
[152] Lyne, A.G., Manchester, R.N., Lorimer, D.R., Bailes, M., d'Amico, N., Tauris, T.M., Johnston, S., Bell, J.F., and Nicastro, L., "The Parkes Southern Pulsar Survey - II. Final Results and Population Analysis", Mon. Not. R. Astron. Soc., 295, 743-755, (1998). 2.4.4, 3.3.1, 4.6

[153] Lyne, A.G., Manchester, R.N., and Taylor, J.H., "The Galactic Population of Pulsars", Mon. Not. R. Astron. Soc., 213, 613-639, (1985). 3.3 .2

[154] Lyne, A.G., Mankelow, S.H., Bell, J.F., and Manchester, R.N., "Radio pulsars in Terzan 5", Mon. Not. R. Astron. Soc., 316, 491-493, (2000). 6,7

[155] Lyne, A.G., and McKenna, J., "PSR 1820-11: A binary pulsar in a wide and highly eccentric orbit", Nature, 340, 367-369, (1989). 2.4.3, 7

[156] Lyne, A.G., and Smith, F.G., Pulsar Astronomy, (Cambridge University Press, Cambridge, 1998), 2nd edition. 2.3, 2.7, 3.5

[157] Manchester, R.N., "Finding Pulsars at Parkes", (September, 2000), [Online Los Alamos Preprint]: cited on 19 March 2001, http://arxiv.org/ abs/astro-ph/0009405. Proc. Astron. Soc. Australia, in press (2001). 1.1

[158] Manchester, R.N., and Lyne, A.G., "Pulsar Interpulses - two poles or one?", Mon. Not. R. Astron. Soc., 181, 761-767, (1977). 1

[159] Manchester, R.N., Lyne, A.G., Camilo, F., Kaspi, V.M., Stairs, I.H., Crawford, F., Morris, D.J., Bell, J.F., and d'Amico, N., "Timing the Parkes Multibeam Pulsars", in Kramer, M., Wex, N., and Wielebinski, R., eds., Pulsar Astronomy - 2000 and Beyond, Astronomical Society of the Pacific Conference Series, 49-54. Astronomical Society of the Pacific, (2000). 2.6.2, 7

[160] Manchester, R.N., Lyne, A.G., d'Amico, N., Bailes, M., Johnston, S., Lorimer, D.R., Harrison, P.A., Nicastro, L., and Bell, J.F., "The Parkes Southern Pulsar Survey I. Observing and data analysis systems and initial results", Mon. Not. R. Astron. Soc., 279, 1235-1250, (1996). 3.1.2, 4.6

[161] Manchester, R.N., Newton, L.M., Cooke, D.J., and Lyne, A.G., "Detection of a pulsar in a long-period binary system", Astrophys. J., 236, L25-L27, (1980). 7

[162] Manchester, R.N., and Taylor, J.H., "Period irregularities in pulsars", Astrophys. J., 191, L63-L65, (1974). 4.3

[163] Manchester, R.N., and Taylor, J.H., Pulsars, (Freeman, San Francisco, 1977). 2.4.1, 2.7

[164] Max Planck Institute for Radio Astronomy, "The EPN Data Archive", (March, 2001), [Online Resource]: cited on 19 March 2001, http://www. mpifr-bonn.mpg.de/div/pulsar/data. 4

Living Reviews in Relativity (2001-5)

http://www. livingreviews .org 
[165] Max Planck Institute for Radio Astronomy, "Home page of the MPIfR pulsar group", (March, 2001), [Online Resources]: cited on 20 March 2001, http://www.mpifr-bonn.mpg.de/div/pulsar/. 2.7

[166] Max Planck Institute for Radioastronomy, "The TIMAPR timing package", (March, 2001), [Available Software]: cited on March 19 2001, http://www.mpifr-bonn.mpg.de/div/pulsar/former/olegd/ soft.html. 4.7

[167] Michel, F.C., Theory of Neutron Star Magnetospheres, (University of Chicago Press, Chicago, 1991). 2.7, 3.5

[168] Middleditch, J., and Kristian, J., "A search for young, luminous optical pulsars in extragalactic supernova remnants", Astrophys. J., 279, 157161, (1984). 3.1 .3

[169] Morrison, L.V., and Ward, C.G., "An analysis of the transients of Mercury: 1677-1973", Mon. Not. R. Astron. Soc., 173, 183-206, (1975). 4.5

[170] Narayan, R., "The birthrate and initial spin period of single radio pulsars", Astrophys. J., 319, 162-179, (1987). 3.2 .1

[171] Narayan, R., and Vivekanand, M., "Evidence for evolving elongated Pulsar Beams", Astron. Astrophys., 122, 45-53, (1983). 3.2.3, 16

[172] NASA and Harvard-Smithsonian Center for Astrophysics, "The NASA Astrophysics Data System", [Online Database]: cited on 19 March 2001, http://adsabs.harvard.edu/. 6.1

[173] National Astronomy and Ionosphere Center, "Pulsars at Arecibo", (December, 2000), [Online Resources]: cited on 7 December 2000, http: //www.naic.edu/ ${ }^{\sim}$ pulsar. 2.7

[174] National Astronomy and Ionosphere Center, "Arecibo Observatory Multibeam Project", (December, 2000), [Online Resources]: cited on 7 December 2000, http://www.naic.edu/ aomulti. 6

[175] Navarro, J., de Bruyn, G., Frail, D., Kulkarni, S.R., and Lyne, A.G., "A very luminous binary millisecond pulsar", Astrophys. J., 455, L55-L58, (1995). 7

[176] Neuhäuser, R., and Trümper, J.E., "On the Number of Accreting and Cooling Isolated Neutron Stars Detected with the ROSAT All-Sky Survey", Astron. Astrophys., 343, 151-156, (1999). 3.3 .3

[177] Nicastro, L., Lyne, A.G., Lorimer, D.R., Harrison, P.A., Bailes, M., and Skidmore, B.D., "PSR J1012+5307: a $5.26 \mathrm{~ms}$ pulsar in a 14.5 hour binary orbit", Mon. Not. R. Astron. Soc., 273, L68-L70, (1995). 2.4.3, 22, 7

[178] Nice, D.J., Two High-Sensitivity Pulsar Searches, PhD Thesis, (Princeton University, Princeton, 1992). 2.5 
[179] Nice, D.J., Sayer, R.W., and Taylor, J.H., "PSR J1518+4904: A Mildly Relativistic Binary Pulsar System", Astrophys. J., 466, L87-L90, (1996). $4.5,7$

[180] Nice, D.J., Sayer, R.W., and Taylor, J.H., "Timing Observations of the J1518+4904 Double Neutron Star System", in Arzoumanian, Z., van der Hooft, F., and van den Heuvel, E.P.J., eds., Pulsar Timing, General Relativity, and the Internal Structure of Neutron Stars, 79-83, (North Holland, Amsterdam, 1999). 7

[181] Nice, D.J., Splaver, E.M., and Stairs, I.H., "On the Mass and Inclination of the PSR J2019+2425 Binary System", Astrophys. J., 549, 516-521, (2001). 7

[182] Nice, D.J., and Taylor, J.H., "PSRs J2019+2425 and J2322+2057 and the Proper Motions of Millisecond Pulsars", Astrophys. J., 441, 429-435, (1995). $\quad 7,7$

[183] Nice, D.J., and Thorsett, S.E., "Pulsar PSR 1744-24A: Timing, Eclipses, and the Evolution of Neutron Star Binaries", Astrophys. J., 397, 249-259, (1992). 7

[184] Pacini, F., "Rotating Neutron Stars, Pulsars, and Supernova Remnants", Nature, 219, 145-146, (1968). 2.1

[185] Peebles, P.J.E., Principles of Physical Cosmology, (Princeton, New Jersey, 1993). 5

[186] Penn State University, "Pulsar Planets", (March, 2001), [Online HTML Document]: cited on 19 March 2001, http://www.astro.psu.edu/ users/pspm/arecibo/planets/planets.html. 1, 2.4.2, 4.7

[187] Peters, P.C., "Gravitational Radiation and the Motion of Two Point Masses", Phys. Rev., 136, 1224-1232, (1964). 3.4

[188] Peters, P.C., and Mathews, J., "Gravitational Radiation from Point Masses in a Keplerian Orbit", Phys. Rev., 131, 435-440, (1963). 3.4

[189] Phillips, J.A., Thorsett, S.E., and Kulkarni, S.R., eds., Planets Around Pulsars, (Astronomical Society of the Pacific Conference Series, 1993). 4.7

[190] Phinney, E.S., "The rate of neutron star binary mergers in the universe: minimal predictions for gravity wave detectors", Astrophys. J., 380, L17L21, (1991). 3.4.1

[191] Phinney, E.S., and Blandford, R.D., "Analysis of the Pulsar $P-\dot{P}$ distribution", Mon. Not. R. Astron. Soc., 194, 137-148, (1981). 3.2 .1

[192] Phinney, E.S., and Sigurdsson, S., "Ejection of pulsars and binaries to the outskirts of globular clusters", Nature, 349, 220-223, (1991). 3.4.1

Living Reviews in Relativity (2001-5)

http://www.livingreviews.org 
[193] Phinney, E.S., and Verbunt, F., "Binary pulsars before spin-up and PSR 1820-11", Mon. Not. R. Astron. Soc., 248, 21P-23P, (1991). 2.4.3, 7

[194] Portegies Zwart, S.F., and Yungelson, L.R., "The possible companions of young radio pulsars", Mon. Not. R. Astron. Soc., 309, 26-30, (1999). 2.4 .3

[195] Prince, T.A., Anderson, S.B., Kulkarni, S.R., and Wolszczan, W., "Timing observations of the 8 hour binary pulsar $2127+11 \mathrm{C}$ in the globular cluster M15", Astrophys. J., 374, L41-L44, (1991). 2.6.4, 3.4.1, 3.4.1, 7

[196] Princeton University, "Home page of the Princeton pulsar group", (March, 2001), [Online HTML Resources]: cited on 20 March 2001, http:// pulsar.princeton.edu/. 2.4.1, 2.7, 12, 4.3, 4.7

[197] Pulsar Group of the Australia Telescope National Facility (ATNF), "Parkes Multibeam Pulsar Survey", (December, 2000), [Online HTML Document]: cited on 2 December 2000, http://www.atnf.csiro.au/ research/pulsar/psr/pmsurv/pmwww. 2.4.1, 2.7

[198] Rankin, J.M., "Toward an empirical theory of pulsar emission. I. Morphological taxonomy", Astrophys. J., 274, 333-358, (1983). 1

[199] Ransom, S.M., Greenhill, L., Herrnstein, J.R., Manchester, R.N., Camilo, F., Eikenberry, S.S., and Lyne, A.G., "A Binary Millisecond Pulsar in Globular Cluster NGC6544", Astrophys. J., 546, L25-L28, (2001). 2.6.4, $3.1 .3,6,7$

[200] Ratnatunga, K.U., and van den Bergh, S., "The rate of stellar collapses in the Galaxy", Astrophys. J., 343, 713-717, (1989). 3.3 .2

[201] Ray, P.S., Thorsett, S.E., Jenet, F.A., van Kerkwijk, M.H., Kulkarni, S.R., Prince, T.A., Sandhu, J.S., and Nice, D.J., "A Survey for Millisecond Pulsars", Astrophys. J., 470, 1103-1110, (1996). 7

[202] Richards, D.W., and Comella, J.M., "The period of pulsar NP 0532", Nature, 222, 551-552, (1969). 2.1

[203] Romani, R.W., "Timing a millisecond pulsar array", in Ögelman, H., and van den Heuvel, E.P.J., eds., Timing Neutron Stars, volume 262 of NATO ASI Series, C: Mathematical and Physical Sciences, 113-117, (Kluwer, Dordrecht, 1989). 5.3

[204] Romani, R.W., "Populations of low-mass black hole binaries", Astrophys. J., 399, 621-626, (1992). 3.5

[205] Romani, R.W., and Taylor, J.H., "An Upper Limit on the Stochastic Background of Ultralow-Frequency Gravitational Waves", Astrophys. J., 265, L35-L37, (1983). 5.1 
[206] Ryba, M.F., and Taylor, J.H., "High Precision Timing of Millisecond Pulsars. I. Astrometry and Masses of the PSR 1855+09 System", Astrophys. $J ., \mathbf{3 7 1}, 739-748$, (1991). $\quad 4.3,4.5$

[207] Ryba, M.F., and Taylor, J.H., "High Precision Timing of Millisecond Pulsars. II. Astrometry, Orbital Evolution, and Eclipses of PSR 1957+20", Astrophys. J., 380, 557-563, (1991). 4.3

[208] Sandhu, J.S., Bailes, M., Manchester, R.N., Navarro, J., Kulkarni, S.R., and Anderson, S.B., "The Proper Motion and Parallax of PSR J0437-4715", Astrophys. J., 478, L95-L98, (1997). 3.3.3, 4.2

[209] Sazhin, M.V., "Opportunities for detecting ultralong gravitational waves", Sov. Astron., 22, 36-38, (1978). 5

[210] Scheuer, P.A.G., "Amplitude variations of pulsed radio sources", Nature, 218, 920-922, (1968). 3.1 .2

[211] Schmidt, M., "Space Distribution and Luminosity Functions of QuasiStellar Radio Sources", Astrophys. J., 151, 393-410, (1968). 3.2.1

[212] Schutz, B.F., "Low-frequency sources of gravitational waves: A Tutorial", in Wilson, A., ed., Proceedings of the 1997 Alpbach Summer School on Fundamental Physics in Space, 229-240. ESA, (1998). 3.4

[213] Schutz, B.F., "Gravitational Waves and Neutron Stars", in Kramer, M., Wex, N., and Wielebinski, R., eds., Pulsar Astronomy - 2000 and Beyond, Astronomical Society of the Pacific Conference Series, 727-732. Astronomical Society of the Pacific, (2000). 3.3 .3

[214] Segelstein, D.J., Rawley, L.A., Stinebring, D.R., Fruchter, A.S., and Taylor, J.H., "New millisecond Pulsar in a Binary System", Nature, 322, 714-717, (1986). 7

[215] Shapiro, S.L., and Teukolsky, S.A., Black Holes, White Dwarfs and Neutron Stars. The Physics of Compact Objects, (Wiley-Interscience, New York, 1983). 2.4.3

[216] Shemar, S.L., and Lyne, A.G., "Observations of pulsar glitches", Mon. Not. R. Astron. Soc., 282, 677-690, (1996). 4.3

[217] Shklovskii, I.S., "Possible causes of the secular increase in pulsar periods", Sov. Astron., 13, 562-565, (1970). 2.4 .4

[218] Smarr, L.L., and Blandford, R.D., "The binary pulsar: Physical processes, possible companions and evolutionary histories", Astrophys. J., 207, 574588, (1976). 2.4 .3

[219] Stairs, I.H., Observations of Binary and Millisecond Pulsars with a Baseband Recording System, PhD Thesis, (Princeton University, Princeton, 1998). 4.1

Living Reviews in Relativity (2001-5)

http://www.livingreviews.org 
[220] Stairs, I.H., Arzoumanian, Z., Camilo, F., Lyne, A.G., Nice, D.J., Taylor, J.H., Thorsett, S.E., and Wolszczan, A., "Measurement of Relativistic Orbital Decay in the PSR B1534+12 Binary System", Astrophys. J., 505, 352-357, (1998). 4.5, 4.5, 7

[221] Stairs, I.H., Lyne, A.G., and Shemar, S.L., "Evidence for free precession in a pulsar", Nature, 406, 484-486, (2001). 3.3 .3

[222] Stairs, I.H., Splaver, E.M., Thorsett, S.E., Nice, D.J., and Taylor, J.H., "A Baseband Recorder for Radio Pulsar Observations", Mon. Not. R. Astron. Soc., 314, 459-467, (2000). For a related online version see: I.H. Stairs, et al., "A Baseband Recorder for Radio Pulsar Observations", (March, 2000), [Online Los Alamos Preprint]: cited on 19 March 2001, http: //arxiv.org/abs/astro-ph/9912272. 4.1

[223] Stairs, I.H., Thorsett, S.E., Taylor, J.H., and Arzoumanian, Z., "Geodetic Precession in PSR B1534+12", in Kramer, M., Wex, N., and Wielebinski, R., eds., Pulsar Astronomy - 2000 and Beyond, Astronomical Society of the Pacific Conference Series, 121-124. Astronomical Society of the Pacific, (2000). 4.6

[224] Standish, E.M., "Orientation of the JPL ephemerides, DE200/LE200, to the dynamical equinox of J 2000", Astron. Astrophys., 114, 297-302, (1982). 4.2

[225] Stappers, B.W., Bailes, M., Lyne, A.G., Manchester, R.N., d'Amico, N., Tauris, T.M., Lorimer, D.R., Johnston, S., and Sandhu, J.S., "Probing the Eclipse Region of a Binary Millisecond Pulsar", Astrophys. J., 465, L119-L122, (1996). 7

[226] Stella, L., Priedhorsky, W., and White, N.E., "The discovery of a 685 second orbital period from the X-ray source $4 \mathrm{U} 1820-30$ in the globular cluster NGC 6624", Astrophys. J., 312, L17-L21, (1987). 3.1 .3

[227] Sternberg Institut at Moscow State University, Relativistic Astrophysics Department, "The Scenario Machine Engine", (March, 2001), [Online Resource]: cited on March 19 2001, http://xray.sai.msu.ru/sciwork/ scenario.html. 3.5

[228] Stinebring, D.R., Ryba, M.F., Taylor, J.H., and Romani, R.W., "Cosmic Gravitational-Wave Background: Limits from Millisecond Pulsar Timing", Phys. Rev. Lett., 65, 285-288, (1990). 5.1

[229] Stokes, G.H., Taylor, J.H., and Dewey, R.J., "A New Binary Pulsar in a Highly Eccentric Orbit", Astrophys. J., 294, L21-L24, (1985). 3.4 .2

[230] Swinburne University of Technology, "Swinburne Astrophysics and Supercomputing Centre", (December, 2000), [Online HTML Resources]: cited on 8 December 2000, http://astronomy. swin.edu.au. 2.7 
[231] Tauris, T. M.and Manchester, R.N., "On the evolution of pulsar beams", Mon. Not. R. Astron. Soc., 298, 625-636, (1998). 3.2.3, 16

[232] Tauris, T.M., and Sennels, T., "Formation of the binary pulsars PSR B2303+46 and PSR J1141-6545. Young neutron stars with old white dwarf companions", Astron. Astrophys., 355, 236-244, (2000). 3.4 .2

[233] Taylor, J.H., "Millisecond Pulsars: Nature's Most Stable Clocks", Proc. IEEE, 79, 1054-1062, (1991). 4.3, 4.7

[234] Taylor, J.H., "Binary Pulsars and Relativistic Gravity", Rev. Mod. Phys., 66, 711-719, (1994). 4.5

[235] Taylor, J.H., and Cordes, J.M., "Pulsar Distances and the Galactic Distribution of Free Electrons", Astrophys. J., 411, 674-684, (1993). 2.3, $2,3,4$

[236] Taylor, J.H., Fowler, L.A., and McCulloch, P.M., "Measurements of General Relativistic Effects in the Binary Pulsar PSR 1913+16", Nature, 277, 437, (1979). 4.5, 4.6

[237] Taylor, J.H., Hulse, R.A., Fowler, L.A., Gullahorn, G.E., and Rankin, J.M., "Further observations of the binary pulsar PSR 1913+16", Astrophys. J., 206, L53-L58, (1976). 4.5

[238] Taylor, J.H., and Manchester, R.N., "Galactic distribution and evolution of pulsars", Astrophys. J., 215, 885-896, (1977). 3.2 .3

[239] Taylor, J.H., and Weisberg, J.M., "A new test of general relativity: Gravitational radiation and the binary pulsar PSR 1913+16", Astrophys. J., 253, 908-920, (1982). $\quad 1,2.4 .3,3.1 .3,4.5,7$

[240] Taylor, J.H., and Weisberg, J.M., "Further experimental tests of relativistic gravity using the binary pulsar PSR 1913+16", Astrophys. J., 345, 434-450, (1989). 1, 2.4.3, 3.1.3, 4.5, 4.5, 4.7, 24, 7

[241] The Nobel Foundation, "The Nobel Prize in Physics 1993", (June, 2000), [Online HTML Document]: cited on 19 March 2001, http://www. nobel. se/physics/laureates/1993. 1, 4.5

[242] The University of Calgary, Radio Astronomy Laboratory, "The Square Kilometre Array Project", (November, 2000), [Online Resource]: cited on 10 December 2000, http://www.ras.ucalgary.ca/SKA. 6

[243] Thorne, K., "A New Window on the Universe: The Search for Gravitational Waves", (October, 1999), [Audio and slides in jpg format]: cited on 8 December 2000, http://www.apscenttalks.org/pres_masterpage. cfm?nameID $=96 . \quad 3.5$

Living Reviews in Relativity (2001-5)

http://www.livingreviews.org 
[244] Thorne, K.S., "Gravitational Waves from Compact Bodies", in van Paradijs, J., van del Heuvel, E.P.J., and Kuulkers, E., eds., Compact Stars in Binaries: IAU Symposium 165, 153-183, (Kluwer, Dordrecht, 1996). 3.3.3, 3.4

[245] Thorsett, S.E., Arzoumanian, Z., F., Camilo, and Lyne, A.G., "The Triple Pulsar System PSR B1620-26 in M4", Astrophys. J., 523, 763-770, (1999). 2.4.2, 4.7, 7

[246] Thorsett, S.E., Arzoumanian, Z., McKinnon, M.M., and Taylor, J.H., "The Masses of Two Binary Neutron Star Systems", Astrophys. J., 405, L29-L32, (1993). 4.5, 7

[247] Thorsett, S.E., Arzoumanian, Z., and Taylor, J.H., "PSR B1620-26: A binary radio pulsar with a planetary companion?", Astrophys. J., 412, L33-L36, (1993). 2.4.2, 4.7, 7

[248] Thorsett, S.E., and Chakrabarty, D., "Neutron Star Mass Measurements. I. Radio Pulsars", Astrophys. J., 512, 288-299, (1999). 4.5

[249] Thorsett, S.E., and Dewey, R.J., "Pulsar Timing Limits on Very Low Frequency Stochastic Gravitational Radiation", Phys. Rev. D, 53, 3468, (1996). 5.1

[250] Toscano, M., Sandhu, J.S., Bailes, M., Manchester, R.N., Britton, M.C., Kulkarni, S.R., Anderson, S.B., and Stappers, B.W., "Millisecond pulsar velocities", Mon. Not. R. Astron. Soc., 307, 925-933, (1999). 7, 7

[251] Trimble, V., "Motions and Structure of the Filamentary Envelope of the Crab Nebula", Astrophys. J., 73, 535-547, (1968). 2.4.4

[252] Tutukov, A.V., and Yungelson, L.R., "The merger rate of neutron star and black hole binaries", Mon. Not. R. Astron. Soc., 260, 675-678, (1993). 3.5

[253] van den Bergh, S., and Tammann, G.A., "Galactic and Extragalactic Supernova Rates", Annu. Rev. Astron. Astrophys., 29, 363-407, (1991). 3.3 .2

[254] van den Heuvel, E.P.J., "The binary pulsar PSR J2145-0750: a system originating from a LMXB with a donor star on the AGB?", Astron. Astrophys., 291, L39-L42, (1994). 2.4.3

[255] van den Heuvel, E.P.J., and Lorimer, D.R., "On the Galactic and Cosmic Merger Rate of Double Neutron Stars", Mon. Not. R. Astron. Soc., 283, L37-L39, (1996). 3.4.1

[256] van Kerkwijk, M., and Kulkarni, S.R., "A Massive White Dwarf Companion to the Eccentric Binary Pulsar System PSR B2303+46", Astrophys. J., 516, L25, (1999). 3.4.2, 22, 7 
[257] van Kerkwijk, M.H., Bergeron, P., and Kulkarni, S.R., "The masses of the millisecond pulsar J1012+5307 and its white-dwarf companion", Astrophys. J., 467, L89-L92, (1996). 2.4.3

[258] van Kerkwijk, M.H., and Kulkarni, S.R., "Spectroscopy of the white-dwarf companions of PSR B 0655+64 and 0820+02", Astrophys. J., 454, L141L144, (1995). 7

[259] Vivekanand, M., and Narayan, R., "A new look at pulsar statistics birthrate and evidence for injection", J. Astrophys. Astron., 2, 315-337, (1981). $\quad 3.2 .1$

[260] Walter, F.M., "The Proper Motion, Parallax, and Origin of the Isolated Neutron Star RX J185635-3754", Astrophys. J., 549, 433-440, (2000). 3.3 .3

[261] Walter, F.M., Wolk, S.J., and Neuhauser, R., "Discovery of a nearby isolated neutron star", Nature, 379, 233-235, (1996). 3.3 .3

[262] Weisberg, J., and Taylor, J.H., "General Relativistic Precession of the Spin Axis of the Binary Pulsar B1913+16: First Two Dimensional Maps of the Emission Beam", in Kramer, M., Wex, N., and Wielebinski, R., eds., Pulsar Astronomy - 2000 and Beyond, Astronomical Society of the Pacific Conference Series, 127-130. Astronomical Society of the Pacific, (2000). 3.4.1, 4.6

[263] Weisberg, J.M., "The Galactic Electron Density Distribution", in Johnston, S., Walker, M.A., and Bailes, M., eds., Pulsars: Problems and Progress, IAU Colloquium 160, 447-454, (Astronomical Society of the Pacific, San Francisco, 1996). 2.7

[264] Weisberg, J.M., Romani, R.W., and Taylor, J.H., "Evidence for geodetic spin precession in the binary pulsar 1913+16", Astrophys. J., 347, 10301033, (1989). 4.6, 24

[265] Wex, N., Kalogera, V., and Kramer, M., "Constraints on Supernova Kicks from the Double Neutron Star System PSR B1913+16", Astrophys. J., 528, 401-409, (2000). 4.6

[266] White, N.E., and Zhang, W., "Millisecond X-ray pulsars in low-mass Xray binaries", Astrophys. J., 490, L87-90, (1997). 2.4 .3

[267] Wijnands, R., and van der Klis, M., "A millisecond pulsar in an X-ray binary system", Nature, 394, 344-346, (1998). 2.4 .3

[268] Will, C.M., "Einstein's Relativity Put to Nature's Test: A Centennial Perspective", (October, 1999), [Audio and slides in jpg format]: cited on 8 December 2000, http://www.apscenttalks.org/pres_masterpage. cfm?nameID=99. 4.7

Living Reviews in Relativity (2001-5)

http://www.livingreviews.org 
[269] Will, C.M., Theory and Experiment in Gravitational Physics, (Cambridge University Press, Cambridge, 1981). 4.7

[270] Wolszczan, A., "A nearby 37.9 ms radio pulsar in a relativistic binary system", Nature, 350, 688-690, (1991). 2.6.1, 3.4.1, 7

[271] Wolszczan, A., "Confirmation of Earth-mass planets orbiting the millisecond pulsar PSR 1257+12", Science, 264, 538-542, (1994). 4.7, 7

[272] Wolszczan, A., Doroshenko, O., Konacki, M., Kramer, M., Jessner, A., Wielebinski, R., Camilo, F., Nice, D.J., and Taylor, J.H., "Timing Observations of Four Millisecond Pulsars with the Arecibo and Effelsberg Radio Telescopes", Astrophys. J., 528, 907-912, (2000). 2.4.2, 4.7, 7

[273] Wolszczan, A., and Frail, D.A., "A planetary system around the millisecond pulsar PSR 1257+12", Nature, 355, 145-147, (1992). 1, 2.4.2, 2.6.1, $4.7,7$

[274] Wolszczan, A., Kulkarni, S.R., Middleditch, J., Backer, D.C., Fruchter, A.S., and Dewey, R.J., "A 110-ms pulsar, with negative period derivative, in the globular cluster M15", Nature, 337, 531-533, (1989). 7 